\title{
Unusual quasars from the Sloan Digital Sky Survey selected by means of Kohonen self-organising maps ${ }^{\star}$
}

\author{
H. Meusinger ${ }^{1}$, P. Schalldach ${ }^{1}$, R.-D. Scholz ${ }^{2}$, A. in der $\mathrm{Au}^{1}$, M. Newholm ${ }^{1}$, A. de Hoon ${ }^{2}$, and B. Kaminsky ${ }^{3}$ \\ 1 Thüringer Landessternwarte Tautenburg, Sternwarte 5, 07778 Tautenburg, Germany \\ e-mail: meus@tls-tautenburg.de \\ 2 Leibniz-Institut für Astrophysik Potsdam (AIP), An der Sternwarte 16, 14482 Potsdam, Germany \\ 3 Universität Leipzig, Fakultät für Physik und Geowissenschaften, Linnéstr. 5, 04103 Leipzig, Germany
}

Received 23 September 2011 / Accepted 3 February 2012

\begin{abstract}
Context. Large spectroscopic surveys have discovered very peculiar and hitherto unknown types of active galactic nuclei (AGN). Such rare objects may hold clues to the accretion history of the supermassive black holes at the centres of galaxies.

Aims. We aim to create a sizeable sample of unusual quasars from the unprecedented spectroscopic database of the Sloan Digital Sky Survey (SDSS).

Methods. We exploit the spectral archive of the SDSS Data Release 7 to select unusual quasar spectra. The selection method is based on a combination of the power of self-organising maps and the visual inspection of a huge number of spectra. Self-organising maps were applied to nearly $10^{5}$ spectra classified as quasars at redshifts from $z=0.6$ to 4.3 by the SDSS pipeline. Particular attention was paid to minimise possible contamination by rare peculiar stellar spectral types. All selected quasar spectra were individually studied to determine the object type and the redshift.

Results. We present a catalogue of 1005 quasars with unusual spectra. These spectra are dominated by either broad absorption lines (BALs; 42\%), unusual red continua (27\%), weak emission lines (18\%), or conspicuously strong optical and/or UV iron emission $(11 \%)$. This large sample provides a useful resource for both studying properties and relations of/between different types of unusual quasars and selecting particularly interesting objects, even though the compilation is not aimed at completeness in a quantifiable sense. The spectra are grouped into six types for which composite spectra are constructed and mean properties are computed. Remarkably, all these types turn out to be on average more luminous than comparison samples of normal quasars after a statistical correction is made for intrinsic reddening $(E(B-V) \approx 0$ to 0.4 for SMC-like extinction). Both the unusual BAL quasars and the strong iron emitters have significantly lower radio luminosities than normal quasars. We also confirm that strong BALs avoid the most radioluminous quasars. For 32 particularly interesting objects, individual spectra are presented. Among these objects are quasars with many narrow BAL troughs and one quasar where the continuum is strongly suppressed by overlapping BAL troughs across nearly the whole SDSS spectrum. Finally, we create a sample of quasars similar to the two "mysterious" objects discovered by Hall et al. (2002, ApJS, 141, 267) and briefly discuss the quasar properties and possible explanations of their highly peculiar spectra.
\end{abstract}

Key words. galaxies: active - quasars: general - quasars: absorption lines - quasars: emission lines - black hole physics

\section{Introduction}

From the very beginning of quasar astronomy (Schmidt 1963; Greenstein \& Matthews 1963; Sandage 1965), optical spectra have been one of the most important sources of information about this class of fascinating objects. Quasars are the most luminous types of active galactic nuclei (AGNs). The average ultraviolet (UV) and optical spectral energy distribution (SED) of AGNs is offen found to be widely consistent with the predictions of the standard model of a geometrically thin and optically thick accretion disc around a massive or supermassive black hole (Shakura \& Sunyaev 1973; Shields 1978; Laor 1990; Blaes et al. 2001). The continuum radiation field from the accretion disc provides the photons for the ionisation of the surrounding gas and powers broad and narrow emission lines. The unified AGN model (Antonucci 1993; Urry \& Padovani 1995) allows us to understand some differences in the spectra of various AGN types as being caused by different orientations of the sightline towards

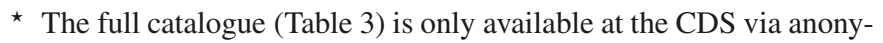
mous ftp to cdsarc.u-strasbg. fr $(130.79 .128 .5)$ or via http://cdsarc.u-strasbg.fr/viz-bin/qcat?]/A+A/541/A77 the central engine. For the vast majority of known quasars, the UV-optical SED is well represented by global composite spectra produced from large samples (e.g., Francis et al. 1991; Zheng et al. 1997; Vanden Berk et al. 2001), where discretion should be exercised however when using the composite of one sample as a template for other samples (Vanden Berk et al. 2001; Richards et al. 2003).

It is to be expected that various predicted spectral features, such as the spectral slope or the strength of broad emission lines, can change from quasar to quasar owing to variations in physical parameters (e.g., Koratkar \& Blaes 1999; Laor \& Davis 2011), and there is empirical evidence that spectral properties can also be modified by variability (Kinney et al. 1990; Wilhite et al. 2005; Hall et al. 2011; Meusinger et al. 2011). Further modifications of the spectrum can be caused by absorption from material related to the quasar itself, to the host galaxy, or from unrelated intervening matter along the line of sight. In addition, some spectral features can be diluted by unrelated emission components (Johnston et al. 2003; Kishimoto et al. 2008) or magnified by gravitational lensing (Abajas et al. 2007; Yonehara et al. 2008; Blackburne et al. 2011). 
On the other hand, it is not to be expected that quasars are fully described by a static model, because "quasars are not things so much as processes" (Richards et al. 2011). Over approximately the past decade, spectroscopic surveys have revealed a variety of quasar spectra that dramatically differ from the standard SED, and confirmed the existence of populations of exotic and hitherto unknown quasar types (Fan et al. 1999; White et al. 2000; Menou et al. 2001; Hines 2001; Hall et al. 2002, 2004; Brunner et al. 2003; Meusinger et al. 2005; Plotkin et al. 2008; Diamond-Stanic et al. 2009; Urrutia et al. 2009). The discovery and investigation of these rare peculiar objects is important because they may represent links to special evolutionary stages and provide an excellent laboratory to study feedback mechanisms between star formation and accretion activity. There are strong indications from different lines of thought for a tight relationship between the activity and growth of massive black holes and the evolution of their host galaxies (e.g., Sanders et al. 1988; Magorrian et al. 1998; Kauffmann \& Hähnelt 2000; Taniguchi 2003; Häring \& Rix 2004; Hopkins et al. 2006; Kauffmann \& Heckman 2009; Meng et al. 2010; Shabala et al. 2011). Much attention has been focused in particular on identifying the youthful quasar population.

There are several tentative arguments supporting the view that young quasars are associated with gaseous outflows and absorption by dust and gas. Many AGNs show blueshifted absorption from outflowing matter (Ganguly \& Brotherton 2008). Very broad absorption lines (BALs) corresponding to a wide variety of velocities are observed in luminous quasars. These BALs are naturally explained as footprints of powerful, sub-relativistic (up to $\sim 0.2 c$ ) outflows (Weymann et al. 1991), which are perhaps related to radiation-driven winds from the accretion disc (Murray \& Chiang 1998). Broad absorption lines are thus the most obvious manifestation of matter accelerated by the central engine of AGNs. It has been frequently argued that the BAL fraction can be explained as being caused by an orientation effect within the unification model where otherwise normal quasars are seen at sightlines close to the equatorial plane of the AGN (Weymann et al. 1991). Typical BAL quasars are often subdivided into those showing broad absorption lines from only high-ionisation species such as $\mathrm{C}_{\mathrm{IV}}$ and $\mathrm{N}$ v (HiBALs) and those showing in addition also absorption from low-ionisation species such as $\mathrm{Mg}$ II and Al III (LoBALs). A rare subclass of the LoBAL quasars are the FeLoBALs with absorption from metastable excited states of $\mathrm{Fe}_{\mathrm{II}}$ and Fe III (Hazard et al. 1987; Becker et al. 2000). The BAL quasars are thought to account for between $10 \%$ and $\sim 40 \%$ of all quasars (Hewett \& Foltz 2003; Reichard et al. 2003; Carballo et al. 2006; Trump et al. 2006; Dai et al. 2008; Gibson et al. 2009; Allen et al. 2011). We note that the exact BAL fraction is not trivial to determine because of differential selection effects between BAL quasars and non-BAL quasars. In addition, different definitions of BAL quasars have been used in different studies. According to the criteria applied by Trump et al. (2006), BAL quasars comprise $26 \%$ of quasars, LoBALs about $1.3 \%$, and FeLoBALs about $0.3 \%$ (these being the raw fractions without correction for the additional reddening of BAL quasars compared to non-BALs).

Outflows are believed to be fundamental to understanding both the overall picture of AGNs and the AGN feedback. Despite their importance, BAL quasars remain poorly understood. In particular, there is currently no concensus about the role of evolutionary effects in determining the properties of BAL quasars. The properties of HiBAL quasars appear to be consistent with the pure orientation model (e.g., Surdej \& Hutsemekers 1987; Gallagher et al. 2007; Doi et al. 2009). On the other hand,
LoBAL quasars may not be explained by orientation alone (e.g., Ghosh \& Punsly 2007; Montenegro-Montes et al. 2009; Zhang et al. 2010; but see Gallagher et al. 2007; Hall \& Chajet 2011). Their properties probably depend on both orientation and evolution effects (Richards et al. 2011; Allen et al. 2011). Based on the shape of the continuum in the UV and X-rays and on the BAL fraction in the infrared, it has been suggested that FeLoBAL quasars in particular probe a much more obscured quasar population than non-BAL quasars and may represent a young evolutionary stage when a thick shroud of gas and dust is being expelled from the central region of the AGN (Voit et al. 1993; Becker et al. 1997; Canalizo \& Stockton 2001; Lípari et al. 2009; Dai et al. 2010; Farrah et al. 2010).

It was suggested by early studies that AGNs spend a substantial fraction of their lifetimes in a dust-enshrouded environment. This picture was based on the scenario of merger-driven AGN activity (e.g., Sanders et al. 1988; Canalizo \& Stockton 2001; Komossa et al. 2003; Hopkins et al. 2005, 2006; Guyon et al. 2006; Bennert et al. 2008). Major mergers of gas-rich galaxies are very efficient in driving gas and dust to the central regions thus feeding both circumnuclear starbursts and accretion onto massive black holes (e.g., Hernquist 1989; Springel et al. 2005). The natural consequences are not only a connection between nuclear accretion activity and starbursts but also the prediction of a substantial fraction of very dusty quasars. Studies in the radio, infrared, and hard X-ray domain (e.g. Webster et al. 1995; Cutri et al. 2001; White et al. 2003; Glikman et al. 2007; Leipski et al. 2008; Polletta et al. 2008) have revealed a large population (perhaps $>50 \%$ ) of dust-reddened quasars that optical colour surveys tend to miss and that are not fully explained by obscuration in the framework of the (static) unified model (Martínez-Sansigre et al. 2006; Georgakakis et al. 2009; Urrutia et al. 2009). Though it is much more difficult to find dustobscured quasars in the optical, it is also not unrealistic to expect moderately reddened quasars in optical surveys.

Another category of unusual quasar spectra that has been discovered by large spectroscopic surveys, typically display the unobscured UV quasar continuum but extremely weak or undectable emission lines. The first such quasars were detected more than one decade ago (McDowell et al. 1995; Fan et al. 1999). About 80 luminous sources of this type with $2.2 \leq z \leq 5.9$ have since been identified by the Sloan Digital Sky Survey (Shemmer et al. 2010, and references therein). Various suggestions have been made to explain the absence of broad lines in unobscured quasars (e.g., Shemmer et al. 2010; Laor \& Davis 2011, and references therein) but this AGN type remains puzzling. A subset of quasars with weak UV emission lines have been identified that are known as PHL 1811 analogs (Leighly et al. 2001; Leighly et al. 2007; Wu et al. 2011, 2012). Leighly et al. (2007) suggested that the weak UV emission lines of these objects are due to an unusual soft SED deficient in ionising photons, perhaps due to exceptionally high Eddington ratios (see e.g., Shemmer et al. 2009, and references therein). On the basis of the multi-epoch photometric data of the quasars in the SDSS stripe 82, Meusinger et al. (2011) found that weak-line quasars are less variable than normal. This seems to be consistent with the suggestion (Hryniewicz et al. 2010) that these quasars are in an early evolutionary stage. However, we note that the sample of weak-line quasars with reliable variability data is small and needs to be checked carefully for possible stellar contamination.

Unusually strong Fe II emission is observed for a small fraction of quasars. The Fe II emission extends from the near-UV to the optical region and is often one of the most prominent spectral features. The strength of the optical Fe II emission is one of 
the parameters that control a set of correlations between various emission line properties, known as the Eigenvector 1 (E1, Boroson \& Green 1992). Eigenvector 1 is thought to serve as a surrogate H-R diagram for AGNs (Sulentic et al. 2007). The principal driver of $\mathrm{E} 1$ seems to be the accretion rate, which may be related to the evolutionary state. Strong iron emission is correlated to the occurrence of BAL troughs (Boroson \& Meyers 1992; Zhang et al. 2010).

We finally note that double-peaked broad emission lines are another type of rare feature in quasar spectra (Halpern et al. 2001; Strateva et al. 2003; Luo et al. 2009; Chornock et al. 2010). Strateva et al. (2003) presented a sample of 116 double-peaked Balmer line AGNs (corresponding to $4 \%$ of low- $z$ AGNs). The spectral signature of this subclass is displaced red and blue peaks of the emission lines similar to double-peaked emission lines found in the spectra of cataclysmic variables. Double-peaked emission lines are believed to originate from rotational motion in a relativistic accretion disc. High rotational velocities in combination with a highly inclined disc and strong reddening can alter the AGN spectrum significantly (Hall et al. 2002). To date, the double-peak effect has only been observed in lowionisation lines, while the UV lines, which dominate the spectra of high- $z$ quasars, are usually single-peaked. For some doublepeaked AGNs, a subparsec supermassive black hole binary has been suggested as explanation (Gaskell 1983; Tang et al. 2009). The existence of close massive black-hole binaries appears as a natural consequence of galaxy mergers.

About one decade ago, only a few peculiar quasars had been discovered, mainly from follow-up of radio sources from the FIRST survey (Becker et al. 1995, 2000; White et al. 2000). The Sloan Digital Sky Survey (SDSS; York et al. 2006) demonstrated in its early phase that these rare objects are not unique, but are members of populations of very peculiar quasars that were occasionally found to have spectral properties never seen before (Hall et al. 2002). Meanwhile, the SDSS has dramatically increased the number of spectroscopically confirmed quasars. With its efficient quasar selection technique and huge number of high-quality spectra, the SDSS is uniquely qualified to significantly increase the samples of unusual quasars.

The present paper aims to select spectroscopic outliers among the quasars from the SDSS Seventh Data Release (DR7; Abazajian 2009). The database is shortly described in Sect. 2. The selection itself is based on an artificial neural network algorithm that uses unsupervised learning (Sect.3). The strategy of unusual quasar selection, the rejection of contaminating sources, and the classification of the selected objects are described in Sect. 4. The final catalogue and sample-averaged properties, including composite spectra, are discussed in Sect. 5, followed by the presentation and discussion of 32 particularly strange spectra. Summary and conclusions are given in Sect. 7. Standard cosmological parameters $H_{0}=71 \mathrm{~km} \mathrm{~s}^{-1} \mathrm{Mpc}^{-1}, \Omega_{\mathrm{m}}=0.27$, and $\Omega_{\Lambda}=0.73$ are used throughout the paper.

\section{Database: Sloan Digital Sky Survey}

The Sloan Digital Sky Survey (SDSS; York et al. 2006) mapped one quarter of the sky and performed a redshift survey of galaxies, quasars, and stars. The total spectroscopic survey area covers 9380 square degrees of the high Galactic latitude sky. The spectra were taken with the $2.5 \mathrm{~m}$ SDSS telescope at Apache Point Observatory equipped with a pair of double fibre-fed spectrographs. As the spectra are collected, the data is passed on and filtered through the spectroscopic pipeline. The automated system produces wavelength and flux-calibrated spectra, measures emission and absorption lines, classifies spectra, and determines the redshifts, which are, among other data, written into the headers of the fits files containing the spectra. The spectra are made publicly available via the SDSS Data Archive Server (DAS). The present study uses quasar spectra from the SDSS Seventh Data Release (DR7; Abazajan et al. 2009) available from the DAS. The DR7, marking the completion of the original goals of the SDSS and the end of the phase known as SDSS-II, contains 1440961 spectra in total (after removing skies and duplicates), including 121363 quasar spectra. The spectra cover the wavelength range 3800-9200 A with a resolution of $\sim 2000$ and sampling of $\sim 2.4$ pixels per resolution element.

The majority of quasars were selected for spectroscopic follow-up according to their deviation away from the "stellar locus" in the multidimensional SDSS colour space. The SDSS filter system allows us to discover quasars over the redshift range from $\sim 0$ to 7 . In addition to the multicolour selection, a small fraction of unresolved objects were selected as primary quasar candidates because their SDSS positions closely match either the positions of radio sources from the VLA FIRST survey (Becker et al. 1995) or of ROSAT X-ray sources (Voges et al. 2000). A fully detailed description of the SDSS method of selecting quasars is given by Richards et al. (2002).

A catalogue of 105783 quasars from the SDSS DR7 was compiled and published by Schneider et al. (2010) as the Fifth Edition of the SDSS Quasar Catalogue (hereafter QCDR7). This catalogue illustrates the unprecedented impact that SDSS has made for the quasar database. Nevertheless, since the present study is aimed at the search for very unusual SDSS quasar spectra, our quasar selection is based on the spectra available via the Data Archive Sever of the SDSS DR7 instead of using the QCDR7.

\section{Kohonen self-organising maps}

The self-organising map (SOM) algorithm (Kohonen 1982, 2001) is an example of unsupervised learning of an artificial neural network. It is an effective tool to project higher-dimensional input data onto a two-dimensional (2D) topological map. In this way, the algorithm has the capability of sorting input data onto a plane according to similarity and thus ideally prepares the data for visual inspection. To achieve this goal, each input element is assigned to one neuron in the network. All neurons of the network are organised onto a 2D grid. In each learning step, the input element is reassigned to some neuron, depending on matching criteria described in Sect. 3.2. Through this iterative stochastic process, the neural network is capable of learning, since it is designed to adapt to the input space. Therefore, input elements are not placed randomly, but increasingly closer to similar input elements, thereby clustering the input data.

The characteristic evolution of the Kohonen map of quasar spectra is illustrated in Figs. 1 and 2. About $5 \times 10^{3}$ quasar spectra from the Fourth Data Release (DR4; Adelman-McCarthy et al. 2006) were arranged in 200 iteration steps ${ }^{1}$. The 18 panels show the $2 \mathrm{D}$ arrangement of the spectra at different iteration steps (top: 1, 2, 3, 4, 6, 8, middle: 10, 20, 30, 40, 60, 80, bottom: $100,120,140,160,180,200)$. In Fig. 1, the redshifts $z$ from the spectroscopic pipeline of the SDSS are highlighted by means of colour coding. The gradient from dark to bright (black to yellow

\footnotetext{
1 The only reason for the use of DR4, instead of DR7, is that it can be analysed using substantially less computing time. As this map was produced for illustrative purposes only, we therefore chose to use the smaller database from the DR4.
} 

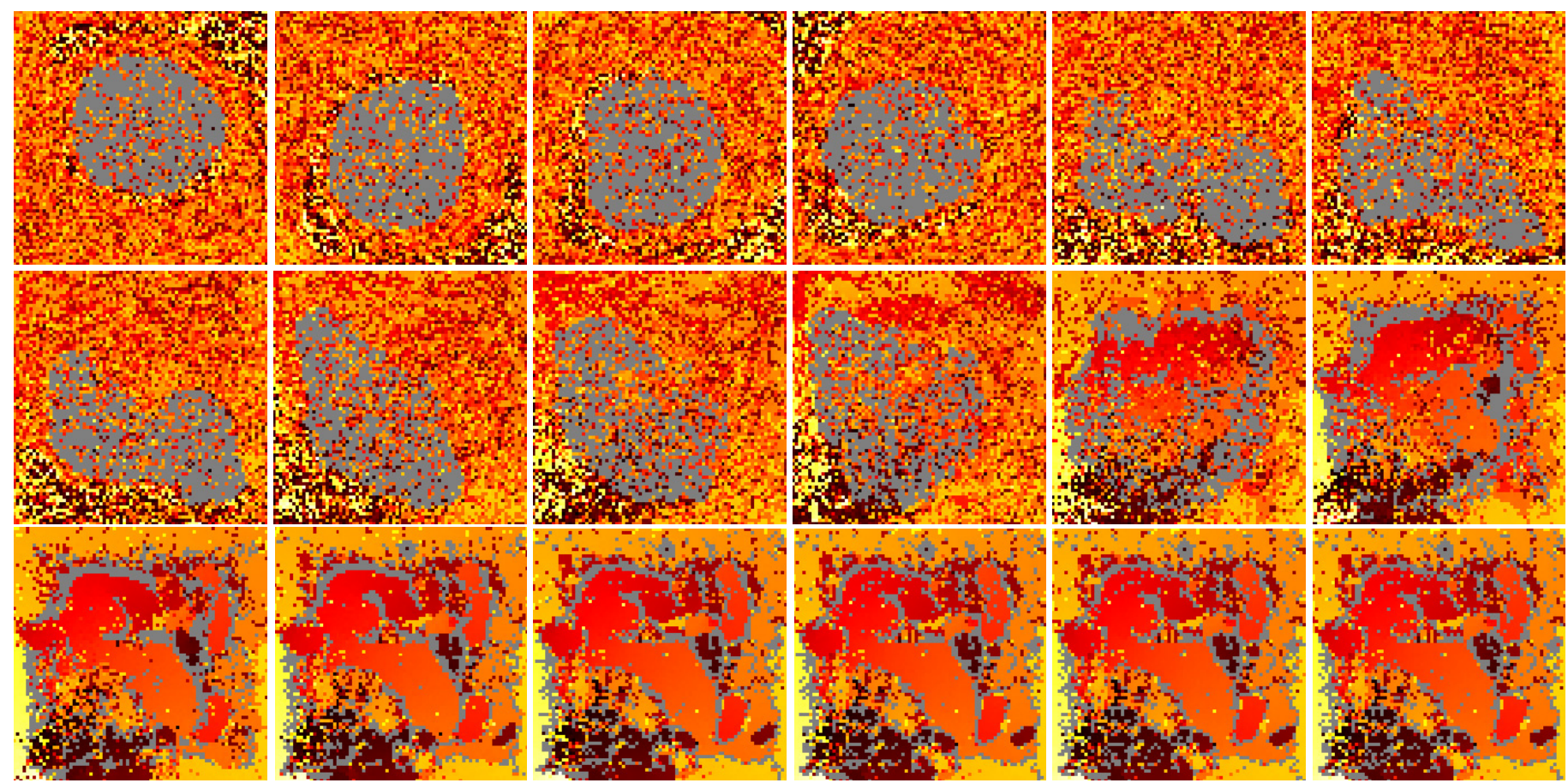

Fig. 1. Temporal evolution (left to right then top to bottom) of the Kohonen SOM of $5 \times 10^{3}$ SDSS quasar spectra. The quasar redshifts are highlighted by means of colour coding. The gradient from dark (black) to bright (light yellow) represents the range from low to high redshift.
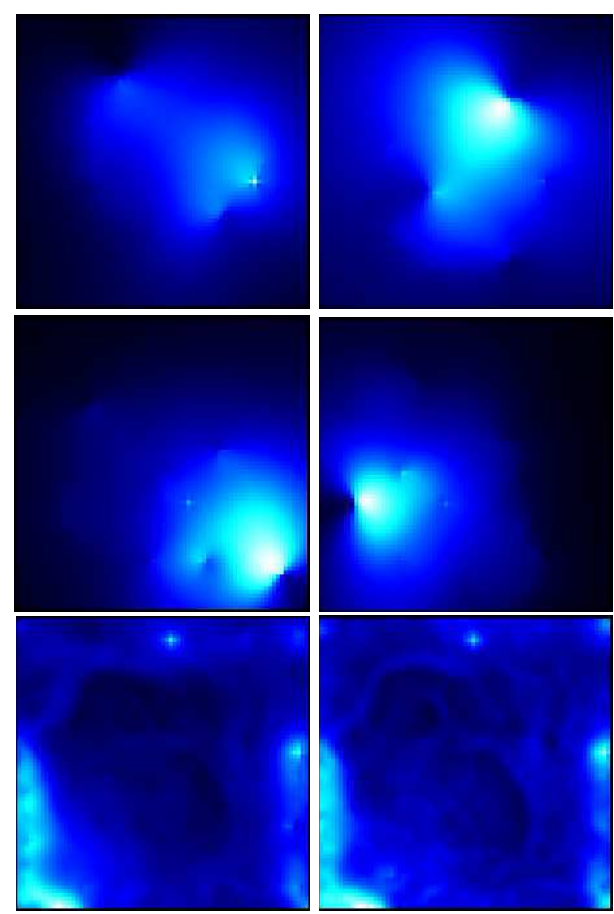
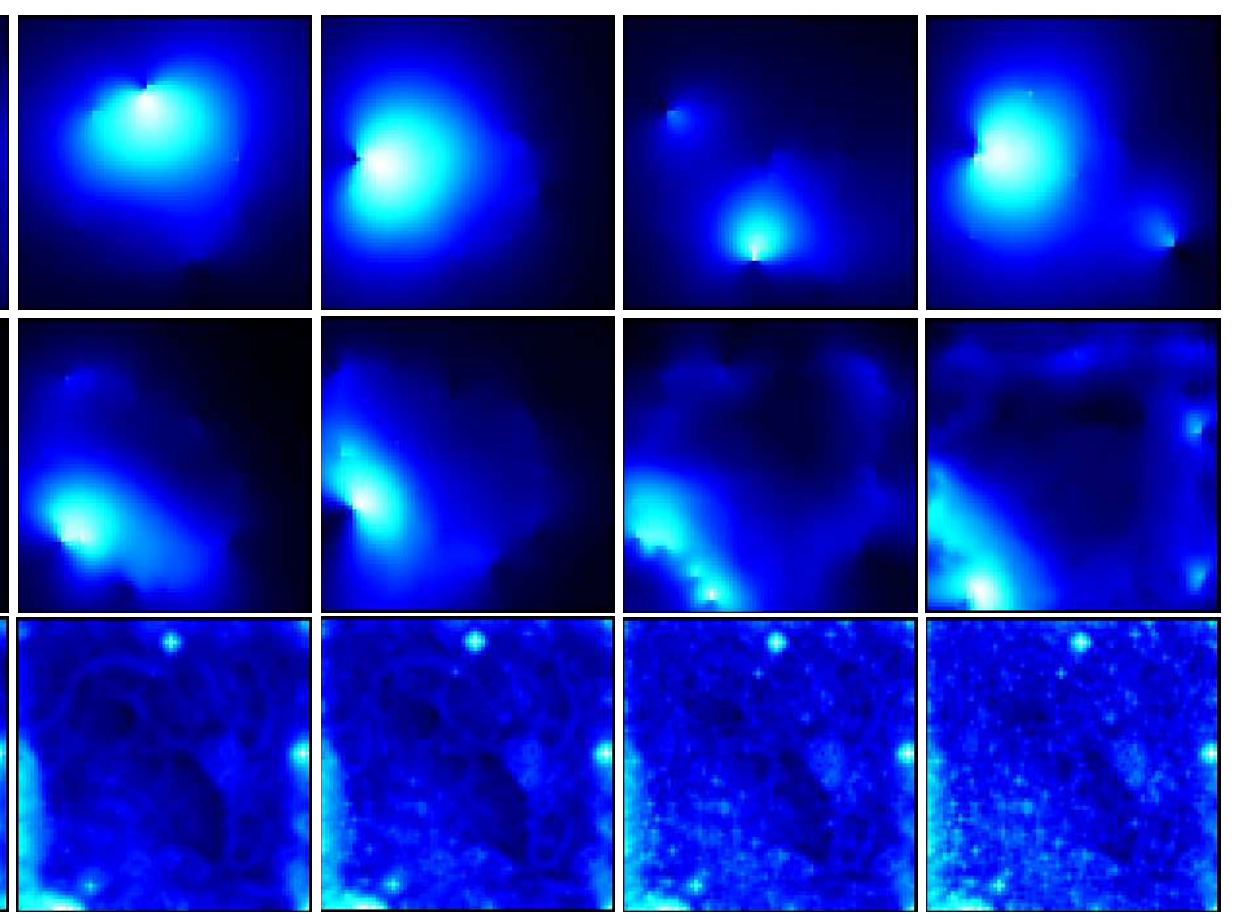

Fig. 2. As Fig. 1, but showing the U-matrices.

in the colour image) represents the range from low to high redshift. About $20 \%$ of the pixels are not associated with spectra and are marked in grey (see below). It is clearly seen that different redshifts are separated in the final Kohonen map.

A usual way of representing a SOM is the unified distance matrix (U-matrix; Ultsch \& Siemon 1990) shown in Fig. 2 for the same iteration steps as in Fig. 1. The U-matrix visualises the differences between the spectra and their neighbours. Light colours indicate high degrees of variation between adjacent spectra. Strong differences between the neighbours on the map are measured, in particular, for rare peculiar spectra. Figure 2 illustrates that these outliers tend to settle at the edges and corners of the final SOM. This is a useful property of the Kohonen maps that allows the efficient selection of unusual spectra.

For the present study, the quasar spectra from the SDSS DR7 were clustered according to their relative differences. At 
a given redshift, these differences are dominated by the shape of the continuum and the presence of strong/broad absorption and emission features. Ideally, the spectra will be sorted according to their spectral subtypes, thus differentiating between quasar subclasses.

In this section we describe the preparation of the spectral dataset and the SOM algorithm in a mathematically handwaving manner. A more detailed description of the method and the visualisation of results from the Kohonen mapping of large samples of spectra will be given elsewhere (in der Au et al., in prep.).

\subsection{Data set}

An SQL query asking for quasars (SPEC_CLN $=3$ or 4 , i.e., quasar or high- $z$ quasar) with redshifts $z>0.5$ in the SDSS DR7 returned 103955 spectra. We are aware of the possibility that some unusual quasar spectra were classified by the SDSS spectroscopic pipeline as unknown objects (spectral class $=$ UNKNOWN, SPEC_CLN $=0$ ). However, the subsample of the UNKNOWNs is quite heterogeneous in nature and contains a high fraction of spectra with low signal-to-noise ratios $(\mathrm{S} / \mathrm{Ns})$. In addition, there are, of course, no reliable redshifts given for these objects. As a consequence, analysing the SOMs of this subsample requires a different approach than for the quasars. The present paper is concerned with the SOMs of the quasar sample (but see Sect.6.2 for an exception). The computation and systematic analysis of the SOMs for the UNKNowns is in preparation and will be the subject of a separate investigation.

As a first step, the overall size of the data set was reduced. We extracted the spectra from the FITs files, together with the selected header keywords Z, SPEC_CLN, MJD, FIBERID, and PLATEID. As our search for unusual quasars was designed to identify spectral peculiarities covering a wide spectral range, such as strong BALs, overlapping absorption troughs, and strong reddening, we reduced the resolution of the spectra used for the Kohonen method by a factor of four, which resulted in $\sim 975$ pixels covering the wavelength range of 3800-9200 $\AA$ in the observer frame. The consequence was a significant gain of computation time. In the subsequent process of evaluating the selected spectra we used, however, the original SDSS spectra. All spectra were normalised to the integrated flux density.

The Kohonen method applied to the whole sample of quasar spectra is expected to separate the spectra according to their redshifts $z$ (Fig. 1). However, we repeat that it was our aim to search for unusual spectra. It is therefore advisable to apply the method to the quasars in narrow $z$ intervals. The bin size $\Delta z$ should be small enough to ensure that the differences between the spectra, as seen by the SOM, caused by their different redshifts are smaller than the differences due to any spectral peculiarities. On the other hand, the intervals must be wide enough to cover a large enough number of spectra. After some trials, we found that $\Delta z \sim 0.1$ is a good choice. Hence, we produced SOMs for the quasar spectra in redshift bins with a 0.1 redshift step size. The mean number of spectra per bin amounts to 2680, ranging from 165 to 6847.

\subsection{SOM algorithm}

The set of input data (i.e. spectra) is defined as vectors $\boldsymbol{x}(j)=$ $\left[\xi_{1}(j), . ., \xi_{n}(j)\right] \in \mathfrak{R}^{n}$, where $n=975$ is the number of pixels in each spectrum and $j$ denotes the index in the sequence of source spectra $j=0 \ldots j_{\max }$ (where $j_{\max }$ strongly varies with $z$ ). The neural network consists of $i \in\{1 \ldots N\}$ neurons, represented by weight vectors $\boldsymbol{m}_{i}=\left[\mu_{i, 1}, \ldots, \mu_{i, n}\right] \in \mathfrak{R}^{n}$, that are organised on a two-dimensional grid. We use a flat grid with closed boundaries (in contrast to cylindrical or toroidal boundary conditions). The weight vectors are modified in each iteration step and thus vary with the discrete time coordinate $t=0,1,2, \ldots, t_{\max }$, i.e., $\boldsymbol{m}_{i}=\boldsymbol{m}_{i}(t)$. Each weight vector $\boldsymbol{m}_{i}$ can be considered as an artificial spectrum. The entire set of weight vectors approximates the distribution of input spectra.

The SOM algorithm is essentially based on two processes that are responsible for the self-organising properties of the neural network: (1) determining the best-matching unit (BMU) for a given randomly chosen input element and (2) the successive adaptation of the weight vectors in the neighbourhood of the BMU towards the given input element.

For a given spectrum $\boldsymbol{x}(j)$, the Euclidean distance $\left\|\boldsymbol{x}(j)-\boldsymbol{m}_{i}\right\|$ to each neuron $\boldsymbol{m}_{i}$ is computed and the BMU is identified with the winning neuron $\boldsymbol{m}_{\mathrm{c}}$, i.e. the neuron for which the distance is minimised, i.e.,

$\left\|\boldsymbol{x}(j)-\boldsymbol{m}_{\mathrm{c}}\right\|=\min _{i}\left\{\left\|\boldsymbol{x}(j)-\boldsymbol{m}_{i}\right\|\right\}$.

In the next iteration step $(t \rightarrow t+1)$, an adaptation of all neurons is performed, according to their difference from a spectrum $\boldsymbol{x}_{j}$ and the neighbourhood function $h_{\mathrm{c}, i}(\tau)$

$\boldsymbol{m}_{i}(t+1)=\boldsymbol{m}_{i}(t)+h_{\mathrm{c}, i}(\tau) \cdot\left[\boldsymbol{x}(j)-\boldsymbol{m}_{i}(t)\right]$,

where $\tau=t / t_{\max }$. The neighbourhood function

$h_{\mathrm{c}, i}(\tau)=\alpha(\tau) \cdot \exp \left(-\frac{\left\|\boldsymbol{r}_{\mathrm{c}}-\boldsymbol{r}_{i}\right\|}{2 \sigma^{2}(\tau)}\right)$

acts as a smoothing kernel over the network and converges to zero with an increasing number of learning steps. The vectors $\boldsymbol{r}_{\mathrm{c}} \in \mathfrak{R}^{2}$ and $\boldsymbol{r}_{i} \in \mathfrak{R}^{2}$ are the location vector of the BMU and and the location vector of the weight vector $\boldsymbol{m}_{i}$, respectively. Compared to the frequently used Gaussian smoothing kernel, Eq. (3) uses a slightly modified version that has broader wings and a sharper peak. We found from various trials that Eq. (3) yields more reliable clustering results.

The process of finding the BMU and adapting the weight vectors is performed for each input spectrum in random order within each learning step. The neighbourhood function is modified over time by the learning rate $\alpha(\tau)$ and the radius function $\sigma(t)$ in such a way that $(a)$ the map develops large-scale structures in the early phase, while $(b)$ finer adjustments occur in later steps. Both functions are assumed to monotonically decrease with time $\tau \in\{0 \ldots 1\}$ and are parametrised in the simple way

$\alpha(\tau)=\alpha_{0}\left(\frac{\alpha_{1}}{\alpha_{0}}\right)^{\tau}, \quad \sigma(\tau)=\sigma_{0}\left(\frac{\sigma_{1}}{\sigma_{0}}\right)^{\tau}$,

where $\alpha_{0}, \alpha_{1}, \sigma_{0}, \sigma_{1}$ are the learning parameters of the Kohonen network with $\alpha_{0} \geq \alpha_{1}$ and $\sigma_{0} \geq \sigma_{1}$. The values used here are listed in Table 1.

The number of neurons in the network must at least correspond to the number of source spectra but can be larger, i.e., $N \geq j_{\max }$. Our experience has shown that better results can be achieved when a certain fraction of positions within the map is not occupied with source spectra. These unoccupied neurons open up space to form cluster boundaries between distinct spectral types, which settle into clearly separated areas of the map. Outlier spectra have enough space to roam the neural landscape. We found that a ratio of $N / j_{\max } \approx 1.2$ represents a 
Table 1. Network parameters used for the clustering.

\begin{tabular}{lc}
\hline \hline Parameter & Values \\
\hline Number of neurons $N$ & $196 \ldots 8281$ \\
Number of iteration steps $t_{\max }$ & 100 \\
Learn radius $\sigma_{0}$ & 1.0 \\
Learn radius $\sigma_{1}$ & 0.125 \\
Learning rate $\alpha_{0}$ & 0.25 \\
Learning rate $\alpha_{1}$ & 0.01 \\
\hline
\end{tabular}

good trade-off. Hence, the grid size varies between $14 \times 14$ and $91 \times 91$ neurons for the highest- $z$ bin and the $1.5<z \leq 1.6$ bin, respectively.

In the beginning of the iteration process at $t=0$, each weight vector $\boldsymbol{m}_{i}(0)$ is initialised with a random input spectrum $\boldsymbol{x}(j)$. The initialisation with purely random weight vectors would require much more learning steps to obtain comparable results. In each iteration step, we start by computing the Euclidean distances for the first input spectrum to all weight vectors within the network. The weight vector with the shortest distance is identified with the BMU for this particular input spectrum. Hence, the input spectrum is moved to its network location for this particular learning step. The BMU and all other weight vectors are then updated according to the neighbourhood function. This process is repeated for all remaining input spectra and over multiple learning steps until convergence is reached, i.e. until the BMUs and the associated input spectra essentially remain at the same positions in successive iteration steps. In our case of relatively small maps, the re-distribution of the spectra reaches sufficient convergence after about 100 iteration steps ${ }^{2}$.

\section{Selection and classification of unusual spectra}

\subsection{Selection of spectra from the SOMs}

Originally, our search for unusual spectra was focused on unusual FeLoBAL quasars. The longest-wavelength lines of the strongest UV iron multiplet, UV $1,(\lambda \leq 2600 \AA)$ is shifted into the spectral window of the SDSS spectra for $z \gtrsim 0.6$. This defines the lower limit for our quasar selection. The upper limit is set at $z \lesssim 4.3$ by the demands that $(a)$ the C IV line should be well within the spectral window and $(b)$ the number of quasars per $z$ bin must not be too small. Therewith, the quasar sample is restricted to the range $z=0.6 \ldots 4.3$. Altogether, 99151 spectra in this $z$ range were analysed with the Kohonen method.

For each of the 37 resulting SOMs, the corresponding grid of spectra was produced in the form of an "icon map", where the spectral resolution was reduced by an order of magnitude. Outlier spectra are clustered at the edges and corners of the SOMs (Sect. 3, Figs. 1 and 2) and are thus easy to select by means of the visual inspection of the icon maps. The selected quasar sample is expected to contain various spectral types. We note, however, that the selection procedure $(a)$ is certainly not free of a subjective bias, $(b)$ does not use a quantitative criterion, and $(c)$ is thus incomplete. The latter is, of course, a direct consequence of the term "unusual" not being defined a priori.

We selected 1575 spectra of 1530 objects, 41 objects from this sample have more than one spectrum in DR7. The selected

\footnotetext{
2 There are two reasons for such a small number of iterations in a neural network. First, our interest is focused on broadband spectral features and the spectral resolution is correspondingly low. Secondly, the final spectra maps are inspected visually. For an automated classification and higher resolution spectra, $t_{\max }$ must be considerably increased.
}

spectra were first corrected for Galactic foreground extinction and then individually checked to estimate $z$ and object type. The Milky Way extinction curve from Pei (1992) was used for the extinction correction with $E(B-V)$ obtained from the "Galactic Dust Extinction Service" 3 of the NASA/IPAC Infrared Science Archive.

While obtaining the redshift from a typical quasar spectrum is mostly straightforward, the situation is different for highly unusual spectra. If prominent emission lines are present and clearly identified, the redshift is estimated as usual by comparing the measured line centres with a catalogue of their restframe wavelengths. The spectral lines were selected manually to account for such effects as noise, broad absorption lines, or artefacts from bright night-sky lines. If the spectrum is not dominated by BAL troughs and emission lines are only rudimentarily present, or not at all, we tried to estimate the redshift by fitting the continuum to that of the SDSS quasar composite spectrum (Vanden Berk et al. 2001). Redshift estimation was approached with particuliar caution for the BAL quasars without clearly identified emission lines but with many narrow absorption troughs or overlapping troughs. Here, we basically follow the approach outlined by Hall et al. (2002). In several cases, redshifts could be estimated only roughly. Dubious as well as uncertain redshifts were flagged: $f_{z}=1$ for likely but not certain, 2 for uncertain due to the lack of redshift indicators, and 3 for uncertain due to low $\mathrm{S} / \mathrm{N}$, respectively. For example, $z$ could not be estimated for three objects with featureless blue spectra, which were flagged therefore $f_{z}=2$.

\subsection{Contamination}

The procedure of individually investigating each spectrum was repeated in three consecutive, independent runs. At the end, 1014 of the selected 1530 objects $(66 \%)$ were included in the (preliminary) catalogue of unusual quasars, $8 \%$ turned out to be stars, another $8 \%$ were classified as galaxies, and $5 \%$ were rejected because of too low $\mathrm{S} / \mathrm{N}$ and/or errors. About $13 \%$ were rejected as (nearly) normal quasars. Some of these quasars were positioned on the SOM in the area of unusual spectra because the assigned redshift from the SDSS pipeline was wrong. Others were simply located in transition regions between unusual and usual spectra but turned out to be, more or less, usual when individually inspected. Objects displaying strong stellar continua and typical low- $z$ absorption and/or narrow emission lines without broad components were simply classified as galaxies and subsequently rejected. Among the remaining 1014 catalogue entries, there are 18 objects for which $z$ is very uncertain or not determined at all $\left(f_{z}>1\right)$.

Most of the rejected stars were of late spectral type, a few others are early-type stars and a small fraction were identified with exotic types. While normal stars were easily recognised based on their typical spectral features and rejected from the database, there remains a risk that some (a few) rather unusual stellar spectra were not correctly identified, e.g., rare types of white dwarfs (WD; Schmidt et al. 1999, 2007; Szkody et al. 2004; Carollo et al. 2006). Figures 3a-g show peculiar spectra that were classified as quasars by SDSS but rejected later on in the present study either during the inspection of the spectra or as a result of proper motion determinations (see below): (a) the superimposition of the spectra of a normal quasar and a late-type star (SDSS J014349.15+002128.3), (b) the WD-M star binary SDSS J100658.64+121133.9 (Heller et al. 2009),

${ }^{3}$ http://irsa.ipac.caltech.edu/applications/DUST/ 

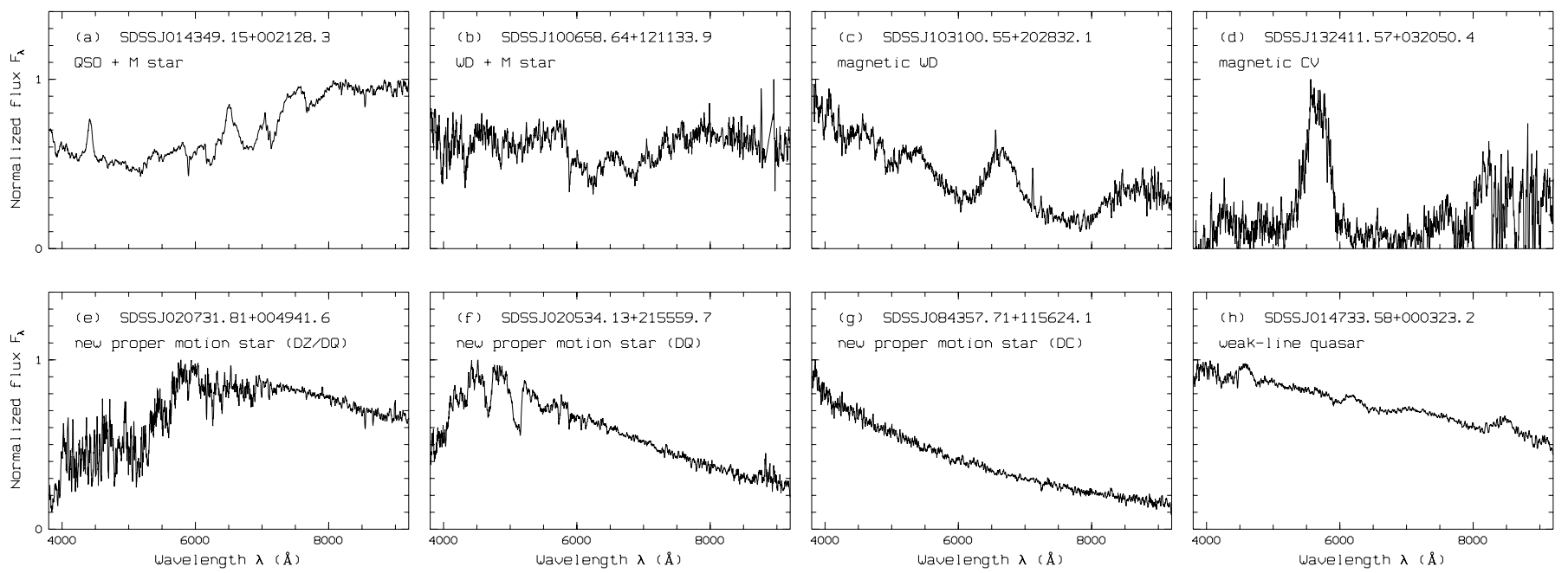

Fig. 3. Examples of unusual spectra that were confirmed (panels a to $\mathbf{g}$ ) or not confirmed (panel h), respectively, as rare types of stellar contaminants in the initially selected quasar sample.

(c) a member of the new class of magnetic WD binaries with extremely low mass transfer rates (SDSS J103100.55+202832.1; Schmidt et al. 2007), (d) a rare magnetic cataclysmic variable with extreme cyclotron features (SDSS J132411.57+032050.4; Szkody et al. 2004), (e) the newly discovered proper motion star SDSS J020731.81+004941.64 (Table 2). In a first attempt, we had classified SDSS J020731.81+004941.6 as a low-redshift FeLoBAL quasar (similar to SDSS J112526.12+002901.3 and SDSS J112828.31+011337.9 from Hall et al. 2002). Its significant proper motion suggests that SDSS J020731.81+004941.6 is a possible extreme DZ or carbon star, (f) the newly discovered proper motion DQ WD SDSS J020534.13+215559.7, and (g) the featureless blue spectrum of a newly discovered proper motion star (SDSS J084357.71+115624.1).

Finally, SDSS J014733.58+000323.2 (Fig. 3 h) is an example of a spectrum that was erroneously rejected in our first analysis attempt. The object was classified as a stellar mass blackhole candidate by Chisholm et al. (2003). Though there is a blue continuum with apparent broad emission-line components, these lines do not fit the positions of the typical broad lines in quasar spectra. Our referee, Dr. Patrick B. Hall, pointed out that there is $\mathrm{C}$ IV $\lambda \lambda$ 1548.20,1550.77 absorption at $z=1.8815$. This high redshift clearly proves that SDSS J014733.58+000323.2 is a quasar. The closer inspection of the spectrum leads to its classification as a slightly reddened $(E(B-V)=0.07$ for SMC-like dust) weakline quasar at $z=1.98$ without $\mathrm{C}$ III] emission and with a shift between the $\mathrm{C}$ IV and $\mathrm{Mg}$ II emission lines.

Stellar contamination remains a serious problem even after the individual inspection of all spectra. Various studies have used astrometric information for the quasar selection (e.g., Sandage \& Luyten 1967; Kron \& Chiu 1981; Meusinger et al. 2002, 2003; Kaczmarczik et al. 2009; Lang et al. 2009). Quasars are sufficiently distant to able to neglect there having any measurable absolute proper motion (pm) in presently available position data. Therefore, absolute $\mathrm{pm}$ are considered helpful data to discriminate quasars from (nearby) stars. We cross-correlate our quasar sample with the PPMXL catalogue (Röser et al. 2010). This catalogue is the largest collection (of nearly one billion objects) of pm in the International Celestial Reference System (ICRS), which is primarily realized by the Hipparcos catalogue.

\footnotetext{
${ }^{4}$ According to the SDSS explorer, a quasar with $z=1.1165$ in DR7 and $z=2.3273$ in DR8, respectively.
}

The PPMXL aims to be complete down to $V \sim 20$, while our quasar sample extends to fainter magnitudes (mean $g$ magnitude $\langle g\rangle=20.02 \pm 1.41)$. With a search radius of $5^{\prime \prime}$, we identified 918 objects $(91 \%)$ from our sample in the PPMXL (mean position difference 0.3 ). We express the probability of significant non-zero $\mathrm{pm}$ in terms of a simple pm index, which is defined as the total $\mathrm{pm} \mu$ in units of the pm error $\epsilon$ :

$I_{\mathrm{pm}}=\sqrt{\left(\mu_{\alpha} \cos \delta\right)^{2}+\mu_{\delta}^{2}} / \sqrt{\epsilon_{\mu_{\alpha} \cos \delta}^{2}+\epsilon_{\mu_{\delta}}^{2}}$.

For the 918 identified objects, we found a mean value of $\left\langle I_{\mathrm{pm}}\right\rangle=$ 2.46 and a median at $0.98 ; 90 \%$ of the objects have $I_{\mathrm{pm}}<2.5$. We restricted the further analysis to the 95 objects with $I_{\mathrm{pm}}>2.5$. One third of this subsample have large pm $\mu>150$ arcsec $\mathrm{yr}^{-1}$, which are likely due to these objects beeing fakes (Röser et al. 2010). We re-investigated the spectra of all 95 pm candidates and searched for possible entries in the SIMBAD 5 and NED $^{6}$ database. For only one object, SDSS J020534.13+215559.7, did the spectrum turn out to be characteristic of a DQ WD (Fig. 3). The star was not found in any stellar database. For 81 objects, the spectra could be assigned to quasars without doubt, another 9 objects are likely quasars. Another four selected pm candidates have relatively featureless spectra, one of them, SDSS J153939.1+274438, is a known radio source with dominant blazar characteristics (Massaro et al. 2009). The large AGN fraction among the pm-selected objects obviously means that the accuracy of the available pm data is insufficient to efficiently select stellar contaminants in the magnitude range of our sample. The trend of increasing $I_{\mathrm{pm}}$ with fainter magnitudes (e.g., $\left\langle I_{\mathrm{pm}}\right\rangle=1.12$ for $g<20$ compared to $\left\langle I_{\mathrm{pm}}\right\rangle=4.10$ for $g>20$ ) and vice versa (e.g., $\langle g\rangle=19.7$ for $I_{\mathrm{pm}} \leq 2.5$ compared to $\langle g\rangle=21.0$ for $\left.I_{\mathrm{pm}}>2.5\right)$ indicates that the pm errors are systematically underestimated at faint magnitudes.

In a second step, we therefore used all available multi-epoch positions from different sky surveys to determine an improved pm. The longest time baseline (with epochs between 1950 and 2002) was provided by the SuperCOSMOS Sky Surveys (SSS; Hambly et al. 2001), including the measurements of overlapping Schmidt plates. In the case of problems with the

\footnotetext{
5 http://simbad.u-strasbg.fr/simbad/

6 http://ned.ipac.caltech.edu
} 

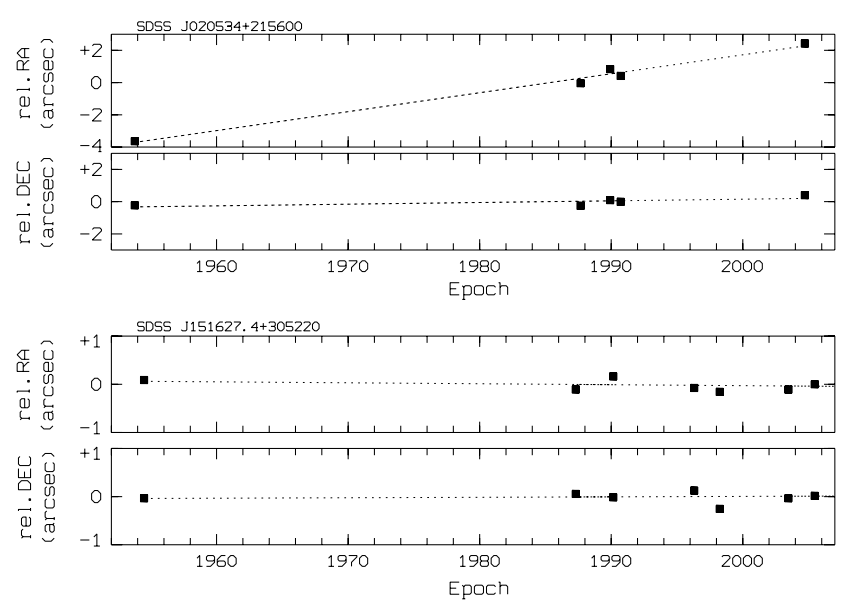

Fig. 4. Two examples of estimates of the proper motions of uncertain quasar candidates: the DQ WD SDSS J020534.13+215559.7 (top) and the quasar SDSS J151627.40+305219.7 (bottom).

SSS measurements on the first epoch Palomar Observatory Sky Survey (POSS1) plates, we used the APM measurements of these POSS1 plates (McMahon et al. 2000). New data (for epochs between 1998 and 2009) came from two data releases of the SDSS (DR7, Abazajian et al. 2009; DR8, Aihara et al. 2011; with 30-70 epochs in the equatorial stripe), the eighth data release of $\mathrm{UKIDSS}^{7}$, and for the brightest objects, from the Carlsberg Meridian Catalog (2006), and the Two Micron All Sky Survey (2MASS; Skrutskie et al. 2006). Finally, we included the epoch 2010 positions of the objects (if measured) in the WISE (Wide-field Infrared Survey Explorer; Wright et al. 2010) preliminary data release. The pm obtained from simple linear fitting of all positions over time were not only judged in terms of their formal errors (typically smaller than the PPMXL errors, if available), but also by the influence of the less-accurate Schmidt plate data. In particular, we did not trust any formally significant $\mathrm{pm}$ if the fit was dominated by only one POSS1 position and could not be confirmed after excluding this plate from the fit. Examples of the proper motion fits are shown in Fig. 4.

The procedure was applied to objects from three subsamples: (a) the 95 pm candidates from the PPMXL, $(b)$ the two "mysterious" objects from Hall et al. (2002) and 15 possibly related objects (Sect.6.2), and (c) an additional 9 objects that had either featureless spectra or spectral features we were unable to interpret. Among these 121 objects are in particular all those with $f_{z}=2$. As expected, most quasar candidates have zero proper motions that we were able to measure very accurately if many epochs were available. However, for seven candidates we found significant proper motions (Table 2). With the exception of SDSS J144354.73+242906.6, the spectra of all these objects are blue and suggest that their classification as WDs with the spectral types given in the last column of Table 2. The spectra of SDSS J134913.51+205646.9 and SDSS J144354.73+242906.6 are noisy; the former is probably a DC and the latter may be a late-type star, but the $\mathrm{S} / \mathrm{N}$ is too low for a reliable type estimation to be possible. Another two unusual quasar candidates, SDSS J032907.24+002438.4 and SDSS J033716.08+000041.7, turned out to be galaxies. These 9 entries were removed so that our catalogue finally contains 1005 entries, among them 14 objects with $f_{z} \geq 2$ that were excluded from the statistical investigations described in Sect. 5 .

\footnotetext{
7 The UKIDSS project is defined in Lawrence et al. (2007).
}

Table 2. Rejected quasar candidates with significant proper motions from the present study.

\begin{tabular}{lcccc}
\hline \hline SDSS J & $\begin{array}{c}\mu_{\alpha} \cos \delta \\
(\mathrm{mas} / \mathrm{yr})\end{array}$ & $\begin{array}{c}\mu_{\delta} \\
(\mathrm{mas} / \mathrm{yr})\end{array}$ & $N_{\mathrm{e}}$ & $T_{\mathrm{WD}}$ \\
\hline $020534.13+215559.7$ & $109.0 \pm 7.2$ & $+10.3 \pm 5.6$ & 5 & $\mathrm{Q}$ \\
$020731.81+004941.6$ & $29.2 \pm 1.0$ & $-6.2 \pm 1.5$ & 39 & $\mathrm{Z}$ \\
$024058.80-003934.5$ & $-10.4 \pm 0.9$ & $-7.5 \pm 1.2$ & 80 & $\mathrm{C}$ \\
$084357.71+115624.1$ & $-9.4 \pm 8.0$ & $+45.8 \pm 6.0$ & 9 & $\mathrm{C}$ \\
$100149.22+144123.8$ & $-345.5 \pm 1.5$ & $-2.3 \pm 3.5$ & 7 & $\mathrm{C}$ \\
$134913.51+205646.9$ & $-68.4 \pm 8.7$ & $-17.6 \pm 3.8$ & 5 & $?$ \\
$144354.73+242906.6$ & $-107.7 \pm 6.2$ & $-112.8 \pm 7.1$ & 6 & $?$ \\
\hline
\end{tabular}

Notes. $N_{\mathrm{e}}$ : number of epochs, $T_{\mathrm{WD}}$ : WD spectral type.

As emphasised above, our method of selecting unusual quasars is not aimed at a complete sample. Nevertheless, to get an idea of the completeness we considered the sample of the unusual BAL quasars from Hall et al. (2002). Among these 23 objects, four were classified by the SDSS DR7 as UNKNOwN (SPEC_CLN=0), and for another three the spectra were unavailable for download. Hence, 16 quasars from this sample are expected to be selected by our approach. Only one Hall quasar is missed, SDSS J121441.42-000137.8, a BAL quasar with possible relatively strong Fe III absorption.

\subsection{Categories of unusual quasar spectra}

According to the different areas populated by unusual quasar spectra in the Kohonen maps, a classification into essentially four categories was immediately suggested. The corresponding dominant spectral features are $(a)$ strong BALs, $(b)$ red continua, $(c)$ weak or absent emission lines, and $(d)$ strong optical or UV iron emission. As a result of evaluating all selected spectra, we decided to subdivide the first category into two types: unusual BAL structures (mostly LoBALs) and more or less normal BAL quasars (mostly strong HiBALs). Moreover, we found that the category of red quasars contains a substantial fraction of spectra where the continuum is significantly red or reddened at UV wavelengths (e.g., $\lambda \lesssim 3000 \AA$ ), but seem to reasonably fit the SDSS quasar composite spectrum at longer wavelengths (see also Hall et al. 2002, their Sect. 6.3.1). Therefore, we split this category into the two types of (pure) red continua and UV-red continua, though it was unclear ab initio whether they represent distinct types. Finally, a relatively small number of objects could not be adequately sorted into one of these six types. These quasars form a small and quite inhomogeneous group, including those that have spectra with exceptionally blue continuum, unusual line profiles, unusual continuum shape (perhaps caused by an error in the flux calibration), or quasars with narrow emission lines but lacking substantial broad emission-line components (type 2 quasar candidates).

Therewith, this paper deals with seven categories or types of unusual quasar spectra (Fig. 5):

A - unusual BAL quasars (mostly LoBAL quasars);

B - quasars with strong but more or less usual BAL structures (mostly HiBALs);

C - quasars where the whole continuum is significantly redder than the SDSS composite;

D - as type C, but strong reddening is obvious only in the UV;

E - quasars with weak or absent emission lines;

$\mathrm{F}$ - quasars with strong iron emission;

M - miscellaneous. 

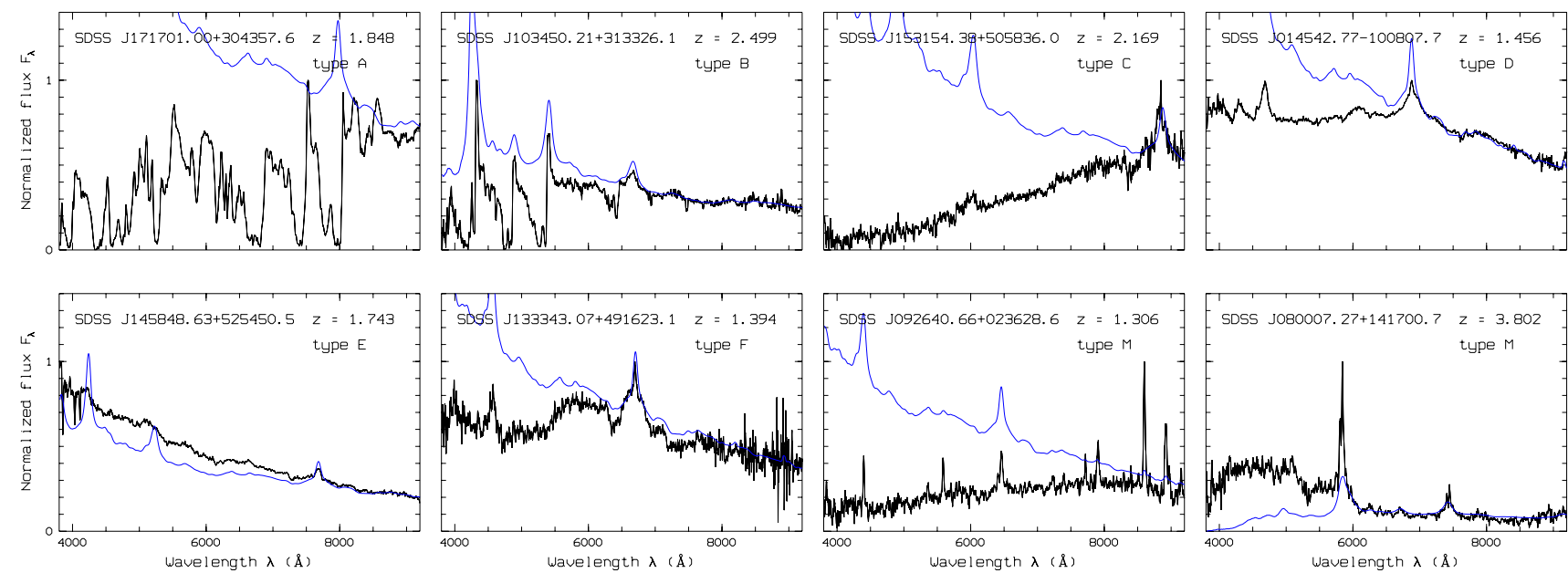

Fig. 5. Example spectra for the unusual quasar types A to M. For comparison, the SDSS quasar composite spectrum (Vanden Berk 2001) is shown, arbitrarily normalised to each object spectrum at the red end.

Since some of the selected spectra appear to be very complex, we decided to build the classification on the subjective impression of the overall picture rather than on quantitative criteria.

The transitions between the various categories are smooth. Many spectra show a mixing of characteristic features of two or three types (see e.g., Fig. 5). Therefore we allocated to each quasar up to three out of the seven possible types $T_{i}, i=1 \ldots 3$ $\left(T_{1} \neq T_{2} \neq T_{3}\right)$, where the significance decreased with $i$. Fortyone percent of the quasars have more than one classification and $8 \%$ have more than two. In particular, there is a strong coupling between unusual BALs, red continua, and strong iron emission. Table 3 lists all three types. In the rest of the paper, we consider the type of highest significance $\left(T=T_{1}\right)$ only.

\subsection{The catalogue}

The results of our visual inspection of the spectra of the 1005 unusual SDSS quasars are given in Table 3. The catalogue was matched with both the QCDR7 (Schneider et al. 2010) and the 08Jul16 version of the catalogue from the FIRST Survey (Becker et al. 1995). The vast majority (98.3\%) of the quasars have entries in the QCDR7 (quasar catalogue flag $f_{c}>0$ ). The agreement between the redshifts from the present study and those from the QCDR7 is generally good ${ }^{8}$. As expected, the discrepancies are larger when the redshifts from the SDSS pipeline are considered: 98 (41) quasars with deviations $>10(50) \%$. The 18 objects missed in the QCDR7 have normal absolute magnitudes and redshifts $\left(\bar{M}_{i}=-25.5, \bar{z}=1.84\right)$ but their redshifts are mostly uncertain $\left(6 / 7 / 4 / 0\right.$ objects have $\left.f_{z}=0 / 1 / 2 / 3\right)$. For these objects, the entries in Cols. 6 and 8 of the catalogue were taken from the SDSS DR7 Explorer.

The full catalogue is available only at the CDS. Table 3 lists the first five entries for guidance regarding its content and form:

Column (1): The running catalogue number.

Column (2): The SDSS J2000 equatorial coordinates for the quasar (taken from the spectrum fits header).

\footnotetext{
${ }^{8}$ For the quasars with certain redshifts $\left(f_{z}=0\right)$, we found six with deviations $>10 \%$. These objects are indicated by $f_{\mathrm{q}}=2$. The deviations were larger than $50 \%$ for SDSS J093437.53+262232.6 and SDSS J131524.00+041734.4.
}

Column (3): The QCDR7 catalogue flag $\left(f_{\mathrm{c}}=0\right.$ : not in QCDR7, 1: in QCDR7 with $z$ deviation $\leq 10 \%, 2$ : in QCDR7 with $z$ deviation $>10 \%$ ).

Columns (4) and (5): The redshift $z$ and the redshift flag $f_{z}$ from the present study $\left(f_{z}=0\right.$ : certain, 1 : likely but not certain, 2: lack of certainly identifiable redshift indicators, 3: low signal-to-noise).

Column (6): The SDSS apparent $i$ band magnitude corrected for Galactic foreground extinction.

Column (7): The absolute $i$ band magnitude, computed as described by Kennefick \& Bursick (2008) for a spectral index $\alpha_{v}=-0.5$.

Columns (8): The FIRST radio detection flag from the QCDR7 (0 for non-detection).

Column (9): The FIRST $1.4 \mathrm{GHz}$ peak flux $F_{1.4}(\mathrm{mJy})$.

Column (10): The radio loudness parameter $R_{i}$ computed from $F_{1.4}$ and the extinction-corrected SDSS $i$ band magnitude following Ivezić et al. (2002).

Column (11): The peculiarity index $\chi^{2}$ of the spectrum (Sect. 5.1).

Columns (12) to (14): The type classifications from the present study (Sect.4.3) in numerical format $(1=\mathrm{A}, 2=$ $\mathrm{B}, \ldots)$ for computational reasons. The priority decreases from $T_{1}$ to $T_{3}$.

Column (15): Comments on spectral peculiarities. Abbreviations:

- abs: absorption;

- ao: associated absorption;

- bel: blueshifted emission line;

- bq: blue quasar;

- em: emission;

- dlas: damped Ly $\alpha$ absorption system;

- fg: foreground;

- lst: longwards-of-systemic trough; 
Table 3. Classification and properties of unusual SDSS quasars.

\begin{tabular}{lcccccccccccccc}
\hline \hline $\mathrm{Nr}$ & SDSS J & $f_{\mathrm{c}}$ & $z$ & $f_{z}$ & $i_{0}$ & $M_{i}$ & $f_{\mathrm{r}}$ & $F_{1.4}$ & $R_{i}$ & $\chi^{2}$ & $T_{1}$ & $T_{2}$ & $T_{3}$ & Comment \\
\hline 1 & $000009.38+135618.4$ & 1 & 2.233 & 1 & 18.17 & -27.45 & 0 & $\ldots$ & $\ldots$ & 2 & 5 & 6 & $\ldots$ & Fe III em? \\
2 & $000326.67+001157.1$ & 1 & 1.845 & 0 & 18.95 & -26.23 & 0 & $\ldots$ & $\ldots$ & 18 & 4 & $\ldots$ & $\ldots$ & \\
3 & $000625.25+154625.8$ & 1 & 1.527 & 1 & 19.58 & -25.16 & 0 & $\ldots$ & $\ldots$ & 77 & 3 & $\ldots$ & $\ldots$ & ao(Mg II); strong [Ne v]; noisy \\
4 & $000728.45-042345.5$ & 1 & 1.198 & 0 & 18.12 & -26.04 & 0 & $\ldots$ & $\ldots$ & 15 & 6 & 1 & $\ldots$ & Fe III em; see 2215(H02) \\
5 & $000920.01+005618.4$ & 1 & 2.387 & 0 & 20.20 & -25.56 & 0 & $\ldots$ & $\ldots$ & 10 & 4 & 5 & $\ldots$ & \\
\hline
\end{tabular}

Notes. Only the first five rows are shown here; the full table is available at the CDS.

Table 4. Mean properties of the unusual quasar types.

\begin{tabular}{lrrccccl}
\hline \hline$T$ & $N$ & $\chi^{2}$ & $z$ & $M_{i}$ & $E_{B-V}^{\text {(intr) }}$ & $M_{i}^{\text {(cor) }}$ & $f_{\mathrm{RL}}$ \\
\hline $\mathrm{A}$ & 215 & 78.8 & 2.02 & -26.3 & 0.18 & -27.6 & 0.26 \\
$\mathrm{~B}$ & 209 & 12.7 & 2.92 & -27.0 & 0.09 & -27.9 & 0.11 \\
$\mathrm{C}$ & 116 & 61.3 & 1.96 & -25.9 & 0.38 & -28.5 & 0.31 \\
$\mathrm{D}$ & 150 & 115.6 & 1.47 & -25.9 & 0.20 & -27.0 & 0.30 \\
$\mathrm{E}$ & 185 & 9.3 & 2.14 & -27.0 & 0.00 & -27.0 & 0.26 \\
$\mathrm{~F}$ & 112 & 28.5 & 1.40 & -26.2 & 0.06 & -26.5 & 0.16 \\
$\mathrm{M}$ & 18 & 13.0 & 1.53 & -25.7 & $\cdots$ & $\cdots$ & 0.33 \\
& & & & & & & \\
all & 1005 & 48.8 & 2.06 & -26.4 & $0.15^{a}$ & $-27.4^{a}$ & 0.23 \\
\hline
\end{tabular}

Notes. ${ }^{(a)}$ Types A to F only.

- mnt: many narrow troughs;

- myst?: related or possibly related to the two mysterious objects; from Hall et al. (2002);

- nlq: narrow-line quasar;

- nt: narrow troughs;

- ot: overlapping troughs;

- sdvdpc: spatially distinct velocity-dependent partial covering; of the continuum source;

- see 0810(H02): see object SDSS J081024.75+ 480615.5; from Hall et al. (2002);

- uc: unusual continuum;

- ulp: unusual line profile.

\section{Mean properties of the various unusual quasar types}

The number of quasars in the seven types are listed in Table 4 along with some mean properties discussed below in this section.

\subsection{Peculiarity index}

As a quantitative measure of the deviation of a given spectrum from the SDSS quasar composite spectrum (Vanden Berk 2001), we computed

$\chi^{2}=\frac{1}{N \sigma^{2}} \sum_{\mathrm{i}=1}^{N}\left[F_{\mathrm{n}}\left(\lambda_{\mathrm{i}}\right)-F_{\text {comp }, \mathrm{n}}\left(\lambda_{\mathrm{i}}\right)\right]^{2}$,

where $F_{\mathrm{n}}$ and $F_{\text {comp,n }}$ are the normalised spectra of the quasar and the composite, respectively, and $\sigma$ is the average noise of $F_{\mathrm{n}}$. The wavelengths $\lambda_{\mathrm{i}}$ refer to the observer frame and the index $i$ indicates the pixel number. The quasar spectrum is normalised to the integrated flux with an integration interval from $4000 \AA$ to $9000 \AA$ for $z<2.29$ and from $1216 \cdot(1+z) \AA$ to $9000 \AA$ for $z \geq 2.29$, respectively. The SDSS quasar composite spectrum was shifted to the redshift $z$ and normalised to match the red end of $F_{\mathrm{n}}(\lambda)$ at $\lambda=8600-9100 \AA$. The noise $\sigma$ was derived from the difference spectrum $D_{\mathrm{n}}(\lambda)=F_{\mathrm{n}}(\lambda)-\tilde{F}_{\mathrm{n}, \mathrm{s} 10}(\lambda)$, where $\tilde{F}_{\mathrm{n}, \mathrm{s} 10}(\lambda)$ is the smoothed version of $F_{\mathrm{n}}(\lambda)$ using a 20 pixel boxcar filter. To reduce the effect of residuals from narrow spectral features, five rest-frame wavelength intervals of $\sim 50 \AA$ width were selected where the contribution from emission lines is small (pseudocontinuum windows; see Sect. 5.5). The average standard devation of $D_{\mathrm{n}}(\lambda)$ in these parts of the spectrum measured in the observer frame is taken as a proxy for $\sigma$. Care has been taken to exclude the regions around the strongest telluric emission lines at $\lambda 5577 \AA$ and $\lambda 6300 \AA$.

At least for the statistical comparison of subsamples, $\chi^{2}$ can be taken as a useful integral measure of the "peculiarity" of the spectrum. The individual values cover the broad range from $\sim 1$ to 5000 with a mean value of $\sim 50$. For comparison, the 15 unusual Hall quasars (Hall et al. 2002) cover the range from 35 to 1089 with a mean value of 261 . When we restrict the selection to $\chi^{2}>35$, we have 296 quasars corresponding to an unusual quasar fraction of $f_{\mathrm{uq}}=296 / 99151=0.0029$ in perfect agreement with $23 / 8000=0.0029$ for the selection by Hall et al. However, if we choose the threshold so that our selected sample has the same mean value of $\chi^{2}$ as the Hall sample, our selection is much less efficient with $f_{\text {uq }}=0.0012$.

The peculiarity index $\chi^{2}$ is particularly sensitive to deviations from the composite spectrum over wide wavelength intervals, i.e., owing to intrinsic reddening or an intrinsically red continuum. It is thus unsurprising that the largest values are measured for the red/UV-red quasars of the types C and D, but also type A. (However, type $\mathrm{C}$ spectra frequently suffer from strong noise, i.e., large values for $\sigma$.) For weak-line quasars, on the other hand, the deviations are restricted mainly to the small wavelength intervals of the lines and thus $\chi^{2}$ is generally small. The unusual BAL quasars of type A have significantly larger values of $\chi^{2}$ than their more normal relatives of type $\mathrm{B}$, in agreement with their stronger apparent intrinsic reddening (see below).

\subsection{Redshifts and absolute magnitudes $M_{i}$}

The first row of Fig. 6 shows the histograms of the $z$ distributions. As can be seen, the different types cover different redshift intervals. This is simply due to the limited spectral window of the observations in combination with the characteristic spectral features being tied to special wavelength intervals. For example, the change in the continuum slope for the type D quasars frequently appears around rest-frame wavelengths between $2000 \AA$ to $3000 \AA$ and is thus best observed for $z \sim 1.5$. At higher $z$, the wavelength of the turnover is shifted towards and beyond the red edge of the spectral window and the spectrum appears to be red (see also Fig. 5). Strong Fe emission in the UV is restricted to the same wavelength interval. Consequently, quasars of types D and $F$ cover similar $z$ intervals. 

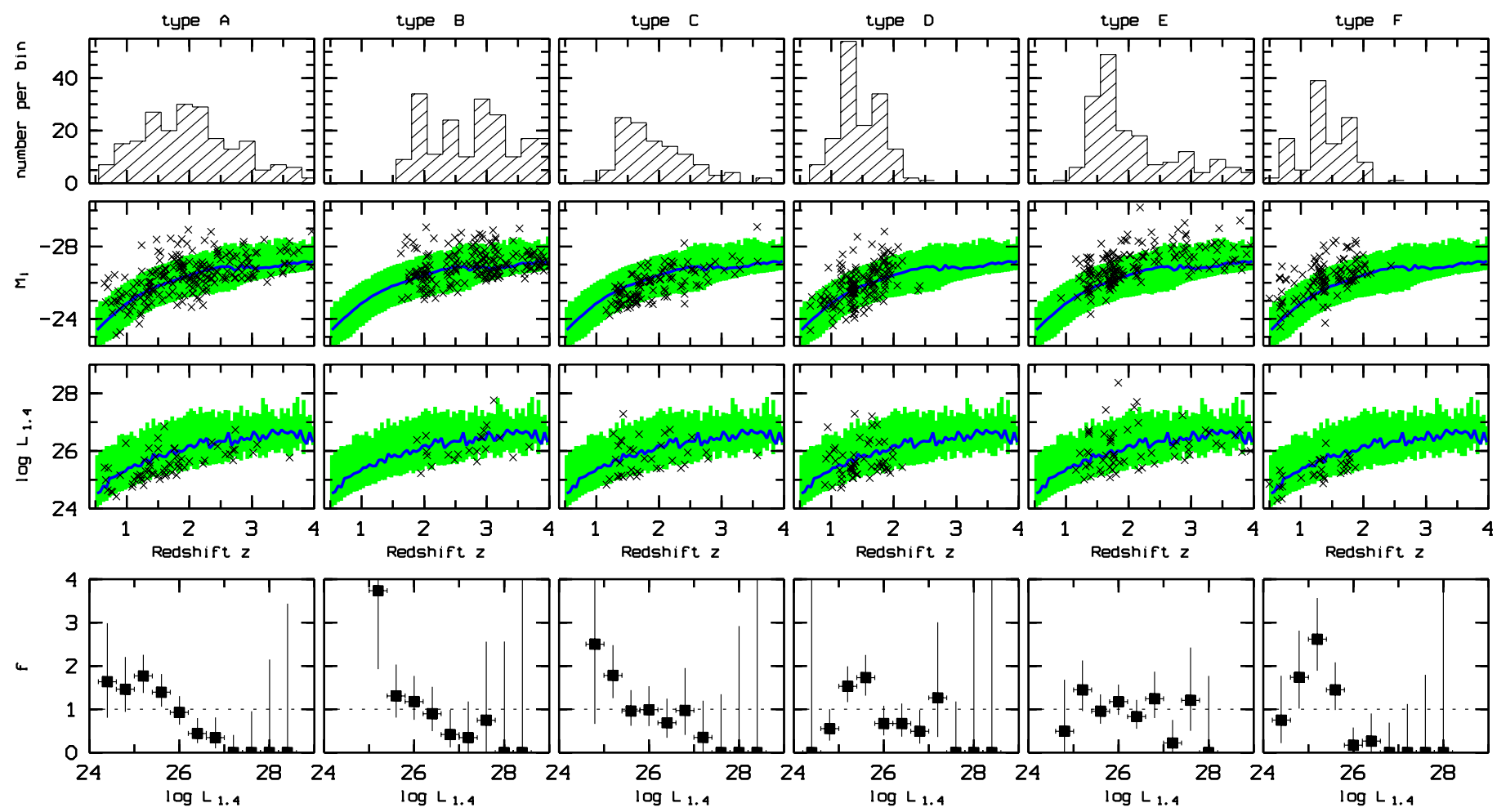

Fig. 6. Top: redshift distribution for types A to F. Second and third row: absolute $i$ magnitude and $1.4 \mathrm{GHz}$ luminosity, respectively, as function of redshift compared with QCDR7. Bottom: relative distribution of the $1.4 \mathrm{GHz}$ luminosity.

In the second row of Fig. 6, the absolute magnitudes $M_{i}$ are plotted as a function of $z$ (crosses), along with the median relation and $1 \sigma$ deviations for the QCDR7 quasars. Owing to the strong inherent redshift bias, the $M_{i}$ distribution of any type cannot be compared with that from the whole QCDR7. For the same reason, the $M_{i}$ distributions of the various quasar types cannot be compared with each other. Therefore, we constructed for each type corresponding comparison samples from the QCDR7 that have identical $z$ distributions. For every quasar (redshift $z$, flag $f_{z}<2$ ), we randomly selected one quasar from the QCDR7 with a redshift in the interval $z-0.05 \ldots z+0.05$. This procedure was performed 100 times to create 100 different comparison samples.

To test whether our unusual quasars and their comparison samples represent the same quasar population with respect to $M_{i}$, we applied the two-tailed two-sample Kolmogorov-Smirnov (KS) test (e.g., Siegel \& Castellan 1988). The null hypothesis $H 0$, that the members of a given type of unusual quasars have the same $M_{\mathrm{i}}$ distribution as the quasars in a comparison sample from QCDR7, is tested against the alternative $H 1$, that the $M_{i}$ distributions of the two samples are different. The KS test uses the maximum difference $D=\max _{i}\left|S_{i}-S_{i \text {, comp }}\right|$ between the cumulative distributions $S_{\text {i }}$ from the two samples. For the twotailed test, $H 0$ has to be rejected at a chosen level $\alpha$ if $D$ is so large that the probability $p$ of its occurrence is $p<\alpha$. Since we have 100 comparison samples, we performed 100 tests per type. Adopting $\alpha=0.05, H 0$ had to be rejected for all comparison samples of types $\mathrm{E}$ and F. For the other types the null hypothesis has to be rejected for only $45 \%$ of the tests for type $\mathrm{C}$ and for $\leq 10 \%$ for the types A, B, and D.

According to Fig. 6, the quasars of types E and F tend to be more luminous than normal. We applied a one-tailed KS test with the alternative hypothesis $H 1$ that unusual quasars are more luminous than usual quasars. Again, $H 0(\alpha=0.05)$ had to be rejected in favour of $H 1$ for the types $\mathrm{E}$ and $\mathrm{F}$. We conclude that both the weak-line quasars and the strong iron emitters are more luminous in the optical than typical quasars from QCDR7. Just et al. (2007) created a sample of 32 of the most luminous quasars from the SDSS DR3 quasar catalogue adopting a minimum luminosity at $M_{i}=-29.28$. Our whole sample includes three quasars brighter than this threshold; all three are of type E, among them the ultra-luminous quasar SDSS J152156.5+520238 with $M_{i}=-30.17$.

It is conceivable that our selection process for spectra with weak emission lines or with strong iron emission might have preferentially selected quasars with higher spectral $\mathrm{S} / \mathrm{N}$, which will be the more luminous ones at each redshift. We estimated $\mathrm{S} / \mathrm{N}$ for the unusual quasar spectra as well as for the spectra of $\sim 10^{4}$ comparison quasars. The noise was measured in defined areas of the spectrum as described in Sect.5.1, the signal was given by the mean flux in the same areas. We indeed found a higher average $\mathrm{S} / \mathrm{N}$ for the unusual quasars namely 11.3 and 15.6 for types E and F, respectively, compared to 8.1 and 9.2 for the corresponding comparison samples. This is, however, not completely unexpected because of the trend of $\mathrm{S} / \mathrm{N}$ with apparent magnitude. In the next step, we created new comparison samples where for each unusual quasar one comparison quasar was identified with similar $z$ (as before) and with similar S/N. For either type $\mathrm{E}$ and $\mathrm{F}$, we found that the absolute magnitudes $M_{i}$ of the unusual quasars of types $\mathrm{E}$ and $\mathrm{F}$ are on average about 0.4 mag brighter than the comparison samples of normal quasars. The $\mathrm{KS}$ test confirms that the differences are significant.

Among all six types, the red quasars of type $C$ have the lowest optical luminosities, as expected. We performed the onetailed KS test to check whether the red quasars are significantly underluminous and found that this is unlikely to be the case. 

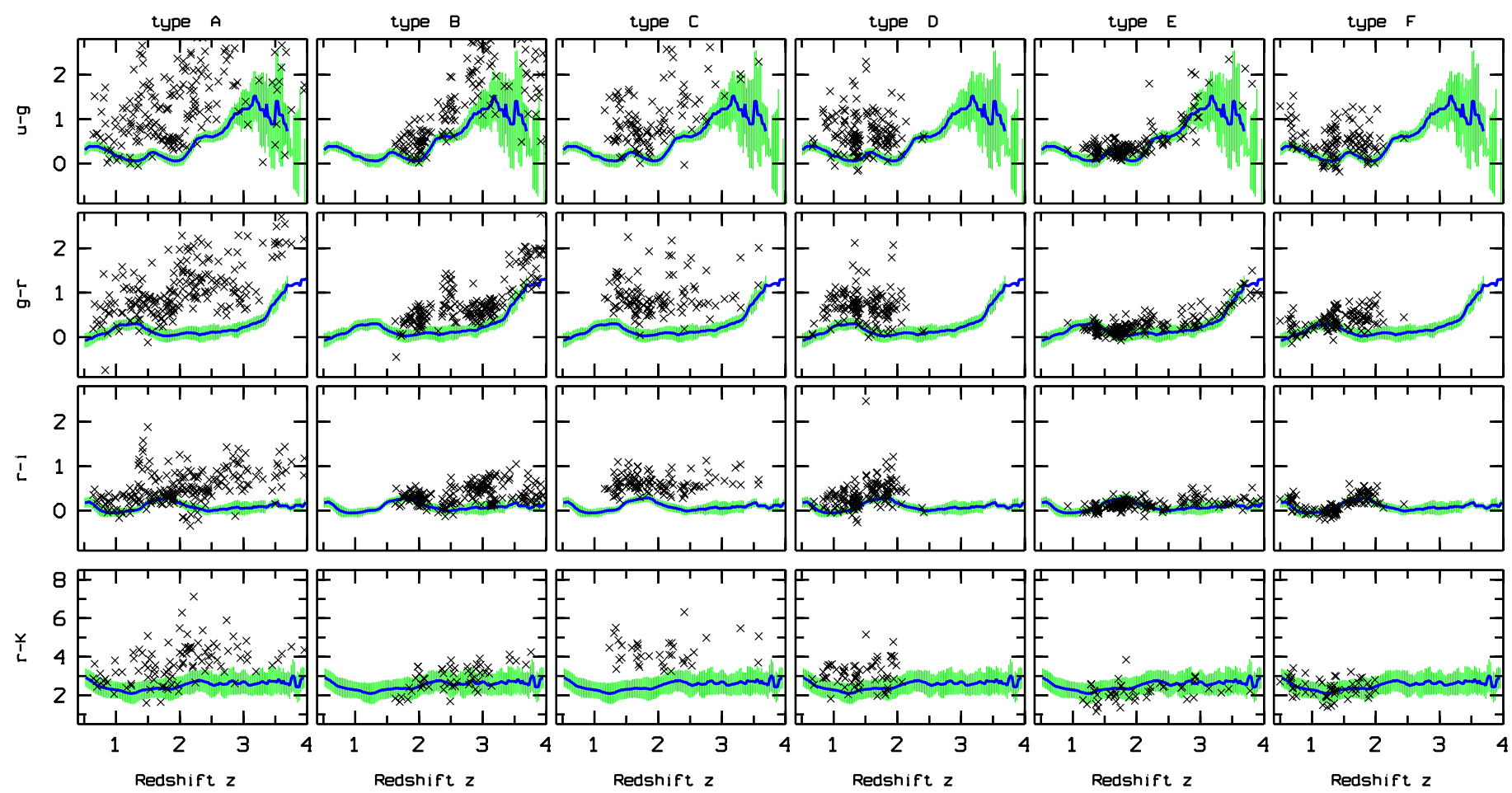

Fig. 7. Colour indices (extinction corrected) as function of redshift for types A to F (top to bottom) in comparison with the SDSS DR7 quasar catalogue (single values and median relations).

\subsection{Radio luminosity and loudness}

The radio detection fraction, $f_{\mathrm{RD}}$, of our whole sample is 0.29 , compared to 0.08 for the quasars from the QCDR7 in the same redshift range $(0.5 \leq z \leq 4.4)$. A similar difference is seen for the fraction $f_{\mathrm{RL}}$ of radio-loud quasars ${ }^{9}$, which is 0.22 in our sample, compared to 0.07 for the QCDR7 quasars. This could imply that independently of colour selection, unusual quasars are detectable over a much longer time span at the FIRST level than normal quasars. Alternatively, these significant differences may be caused by the SDSS having also targeted FIRST sources for spectroscopy when their optical colours did not meet the quasar selection criteria. The FIRST target flag is indeed set for $21 \%$ of the quasars in our sample, compared to $5 \%$ in the whole quasar catalogue.

To test whether the excess radio-detected unusual quasars were only discovered through FIRST targeting, we considered the numbers $N_{\mathrm{F}}$ of quasars with the FIRST target flag set and $N_{\mathrm{C}}$ of quasars with their colour target flag set. We found $N_{\mathrm{F}} / N_{\mathrm{C}}=$ 0.33 for the unusual quasars compared to 0.07 for the QCDR7. A similar result was found when we compared the number $N_{\mathrm{Fs}}$ of quasars selected solely by the FIRST selection (but not the colour selection) to the number $N_{\mathrm{Cs}}$ of quasars without FIRST counterparts but selected solely based on their colours, namely $N_{\mathrm{Fs}} / N_{\mathrm{Cs}}=0.22$ for the unusual quasars and 0.01 for the QCDR7. Moreover, when we binned the unusual quasars into intervals of $\chi^{2}$, it could be clearly seen that the fraction of quasars with their FIRST target flag set increases with the mean peculiarity index (Table 5). Both $N_{\mathrm{F}} / N_{\mathrm{C}}$ and $N_{\mathrm{Fs}} / N_{\mathrm{Cs}}$ rise strongly with $\chi^{2}$ and reach values of $\sim 1$ for the objects showing the strongest mean deviation from the SDSS quasar composite spectrum. We could identify no physical reason for such a trend and concluded

\footnotetext{
9 Using the criterion $R_{i}>1$ as definition for radio loudness; see Ivezić et al. (2002).
}

Table 5. Radio detection fraction for different spectral peculiarities.

\begin{tabular}{lcccccc}
\hline \hline$\chi^{2}$ & $N$ & $N_{\mathrm{F}} / N_{\mathrm{C}}$ & $N_{\mathrm{Fs}} / N_{\mathrm{Cs}}$ & $f_{\mathrm{RD}}$ & $f_{\mathrm{RL}}$ & $f_{\mathrm{RL}} / f_{\mathrm{RD}}$ \\
\hline $0 \ldots 5$ & 248 & 0.17 & 0.09 & 0.19 & 0.18 & 0.94 \\
$5 \ldots 20$ & 333 & 0.20 & 0.15 & 0.23 & 0.16 & 0.72 \\
$20 \ldots 50$ & 184 & 0.34 & 0.22 & 0.28 & 0.23 & 0.82 \\
$50 \ldots 100$ & 123 & 0.69 & 0.41 & 0.48 & 0.38 & 0.80 \\
$100 \ldots 200$ & 61 & 0.73 & 0.62 & 0.48 & 0.39 & 0.83 \\
$>200$ & 42 & 1.16 & 0.91 & 0.55 & 0.31 & 0.57 \\
\hline
\end{tabular}

therefore that the increase in the fraction $f_{\mathrm{RD}}$ of quasars with FIRST detections towards the more peculiar spectra is more likely a selection bias: a large fraction of the unusual quasars were not selected by the colour selection criterion but were targeted by SDSS just because they had been detected as FIRST radio sources.

A similar trend, but weaker, was also observed for the fraction $f_{\mathrm{RL}}$ of the radio-loud quasars. On the other hand, no indication of this trend is seen for the ratio $f_{\mathrm{RL}} / f_{\mathrm{RD}}$. The fraction of radio-loud among the radio-detected quasars for our whole sample is 0.76 , compared to 0.90 for the QCDR7 sample. This means that our selection criterion of peculiar spectra did not induce a substantial bias towards quasars that are more active at radio frequencies than normal.

On the other hand, it is interesting to study whether our lower radio loudness fraction is significant and how it depends on the type properties. For this purpose, we converted the FIRST radio flux densities $F_{1.4}$ to the specific luminosities $L_{1.4}$ emitted at $1.4 \mathrm{GHz}$ (restframe) via

$\log L_{1.4}=23.08+2 \log D_{\mathrm{L}}+\log F_{1.4}-(1+\alpha) \log (1+z)$ 
where $L_{1.4}$ and $F_{1.4}$ are given in $\mathrm{W} \mathrm{Hz}^{-1}$ and mJy, respectively, $D_{\mathrm{L}}$ is the luminosity distance in $\mathrm{Gpc}$, and $\alpha$ is the spectral index $\left(F_{v} \propto v^{\alpha}\right)$, where we assumed that $\alpha=-0.5$. The results are shown in Fig. 6 for the quasars of each type (crosses). The (normal) quasars from the QCDR7 are represented by the median relation and the area populated by $80 \%$ of the quasars closest to the median on either side.

As discussed above for the absolute magnitudes, comparison samples with the same $z$ distribution are needed to check whether the radio luminosities in our sample differ from those of normal QCDR7 quasars. Such comparison samples were constructed in the same way as for $M_{i}$, with the only exception that now only the subsamples of the radio-detected quasars $\left(F_{1.4} \geq 1 \mathrm{mJy}\right)$ were considered. We again first performed the two-tailed KS test with the null hypothesis $H 0$, that there is no difference between the radio luminosities of the unusual quasars of a given type and the comparison sample from the QCDR7, and the alternative $H 1$, that both groups have different radio properties. We found that $H 0$ has to be rejected for all comparison samples of type A and for $80 \%$ of the comparison samples of type F. For the other types, the fraction of rejections is $20 \%$ or less. Since the fraction of radio-loud quasars tends to be smaller for the unusual quasars, we also applied one-tailed tests with $H 1$ : the radio luminosities of the unusual quasars are lower. We found that $H 0$ has to be rejected in favour of $H 1$ for the unusual BAL quasars of type A $(p=0.01)$ and the strong Fe-emitting quasars $(p=0.04)$ of type F. No such firm conclusion can be drawn for the other types.

The bottom row of Fig. 6 shows the distribution of the ratio $f=N\left(L_{1.4}\right) / N_{\mathrm{QC}}\left(L_{1.4}\right)$ where $N\left(L_{1.4}\right)$ is the differential radio luminosity distribution for the corresponding type with equidistant $\log L_{1.4}$ intervals and $N_{\mathrm{QC}}\left(L_{1.4}\right)$ is the distribution averaged over 10 comparison samples from QCDR7, where both distributions are normalised. The vertical bars indicate the propagation of the counting errors of $N\left(L_{1.4}\right)$ and $N_{\mathrm{QC}}\left(L_{1.4}\right)$, which were identified with the Poisson limits at a confidence level of $0.84^{10}$. To compute the upper and lower Poisson levels, the approximate equations given by Gehrels (1986) were applied. It can be clearly seen that strong BALs are not among the most radioloud quasars. The fraction of BAL quasars drops by a factor of at least three between $\log L_{1.4}=25$ and 27. This decline in the fraction of BAL quasars with increasing radio power confirms previous results from Becker et al. (2000) for a smaller sample of BAL quasars and Shankar et al. (2008) for a larger sample based on the catalogue of BAL quasars from Trump et al. (2006). Shankar et al. (2008) argue that such a trend fits well within a simple geometric model. Together with the results in Table 5, the increase in $f$ towards lower $L_{1.4}$ for type A indicates that the real fraction of unusual BAL quasars might be considerably larger than in present samples, where these quasars have instead been targeted mainly as FIRST radio sources.

Finally, we note that there is a similar trend of $f\left(\log L_{1.4}\right)$ for types $\mathrm{B}$ and $\mathrm{C}$. This trend seems to also exist for type $\mathrm{F}$ but is not statistically significant.

\subsection{Optical colours}

Systematic trends of the SED can be illustrated by average spectra but also by the distribution of the colour indices. We first discuss the colours.

Since the colour indices of quasars vary with $z$, it is useful to study colour-redshift diagrams rather than colour-colour

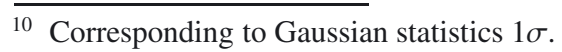

diagrams. Such colour- $z$ diagrams are displayed in Fig. 7 for $u-g, g-r, r-i$, and $r-K$, where $u, g, r, i$ are the SDSS magnitudes from the QCDR7 corrected for Galactic foreground extinction and $K$ is the $K$ band magnitude from the 2MASS survey (Skrutskie et al. 2006). About $60 \%$ of our quasars have 2MASS measurements. We do not see significant differences between the subsamples with or without 2MASS counterparts. As in Fig. 6, the quasars from the QCDR7 are plotted for comparison (median and $1 \sigma$ deviation).

In general, BAL quasars are known to be significantly redder than those without BALs, and LoBAL quasars are even redder (Sprayberry \& Foltz 1992; Reichard et al. 2003; Gibson et al. 2009). It can be clearly seen in Fig. 7 that types A to D tend to have redder colours than the whole SDSS quasar population with strongest deviations for type A. The colours of type $\mathrm{C}$ and D quasars are by definition redder than normal. For type E, the colour-redshift diagrams resemble those of the whole quasar population. The diagrams for type $\mathrm{F}$ quasars indicate a moderate reddening of $u-g$ and $g-r$, while $r-i$ and $r-K$ are approximately the same as for normal quasars.

As shown in Sect. 5.2, types A to D have the same absolute magnitudes as normal quasars. However, if their red colours are due to intrinsic reddening by dust and/or gas, the intrinsic luminosities of these quasars appear to be higher than normal. In Sect. 5.6, we estimate the intrinsic absorption and perform the KS test for the correspondingly corrected $M_{i}$.

\subsection{Composite spectra}

Composite spectra are particularly useful for determining the average spectral properties of quasar samples (Francis et al. 1991; Vanden Berk et al. 2001; Richards et al. 2003). The general procedure of constructing quasar composites is the following: after the redshifts are determined, the individual (foreground) extinction-corrected spectra are rebinned to a common wavelength scale in their restframe, and the quasars are sorted by redshift. Then, generating composites requires essentially two steps: $(a)$ normalisation of the spectra and $(b)$ combining the normalised spectra. Both steps can be performed in different ways.

The normalisation is not entirely trivial because the spectra cover different rest-frame wavelength intervals. To create global composite spectra of normal SDSS quasars, Vanden Berk et al. (2001) started with the arbitrarily scaled spectrum of the lowest redshift quasar (with measured [O III] $\lambda 5007$ line emission), and scaled each subsequent spectrum by the overlap of the preceding average spectrum (i.e., by its mean flux density in the wavelength interval of the overlap). The same procedure was applied in our previous study (Meusinger et al. 2011). Here, however, we followed a slightly different approach. The main reason is that, for our present sample, the flux density of the overlapping interval can be strongly modified by the spectral peculiarities for which we search. Hence, we decided to restrict the normalisation to a few, relatively narrow continuum windows. Though there is no ideal continuum window where a suppression by BALs and/or the contribution from emission lines can be completely ignored, usable pseudo-windows exist however at 3540-3600 ̊, 3030-3090 ̊ (Tsuzuki et al. 2006), and 2000-2300 A (Sameshima et al. 2011). We started by scaling the lowest-redshift spectra. If spectra existed with $z<1.5$, they were arbitrarily normalised to an average flux density $F_{\lambda}=1$ over the wavelength interval $\lambda=3540-3600 \AA$. The thus normalised spectra were combined to a first composite $\boldsymbol{C}_{1}$. Next, the spectra in the redshift range $z=1.5-1.94$ were scaled to 

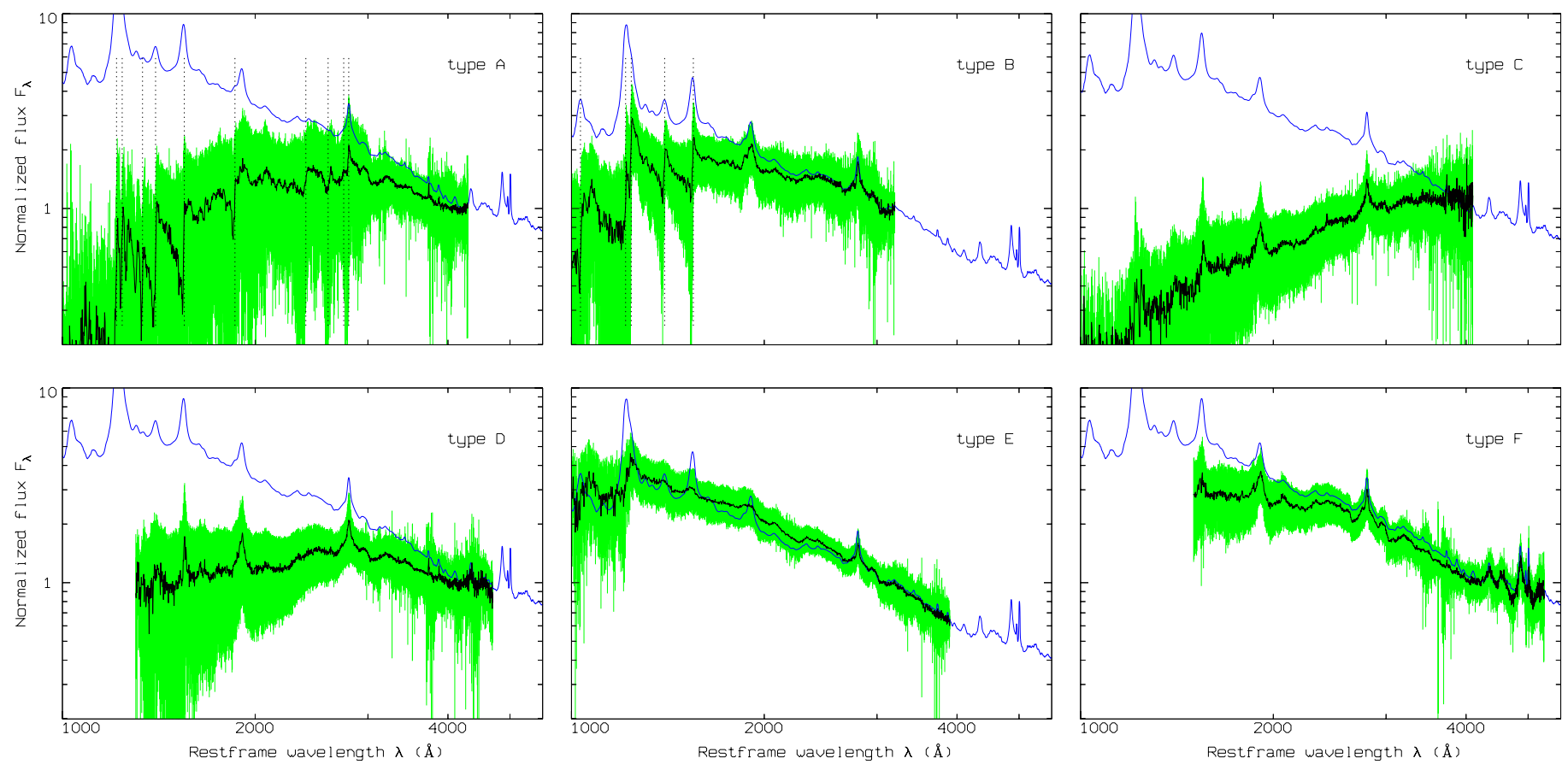

Fig. 8. Arithmetic median composite spectra for types A to F. For comparison the SDSS quasar composite spectrum from Vanden Berk et al. (2001) is shown, arbitrarily normalised at the red end. The dashed vertical lines indicate the strongest absorption lines for types A and B.
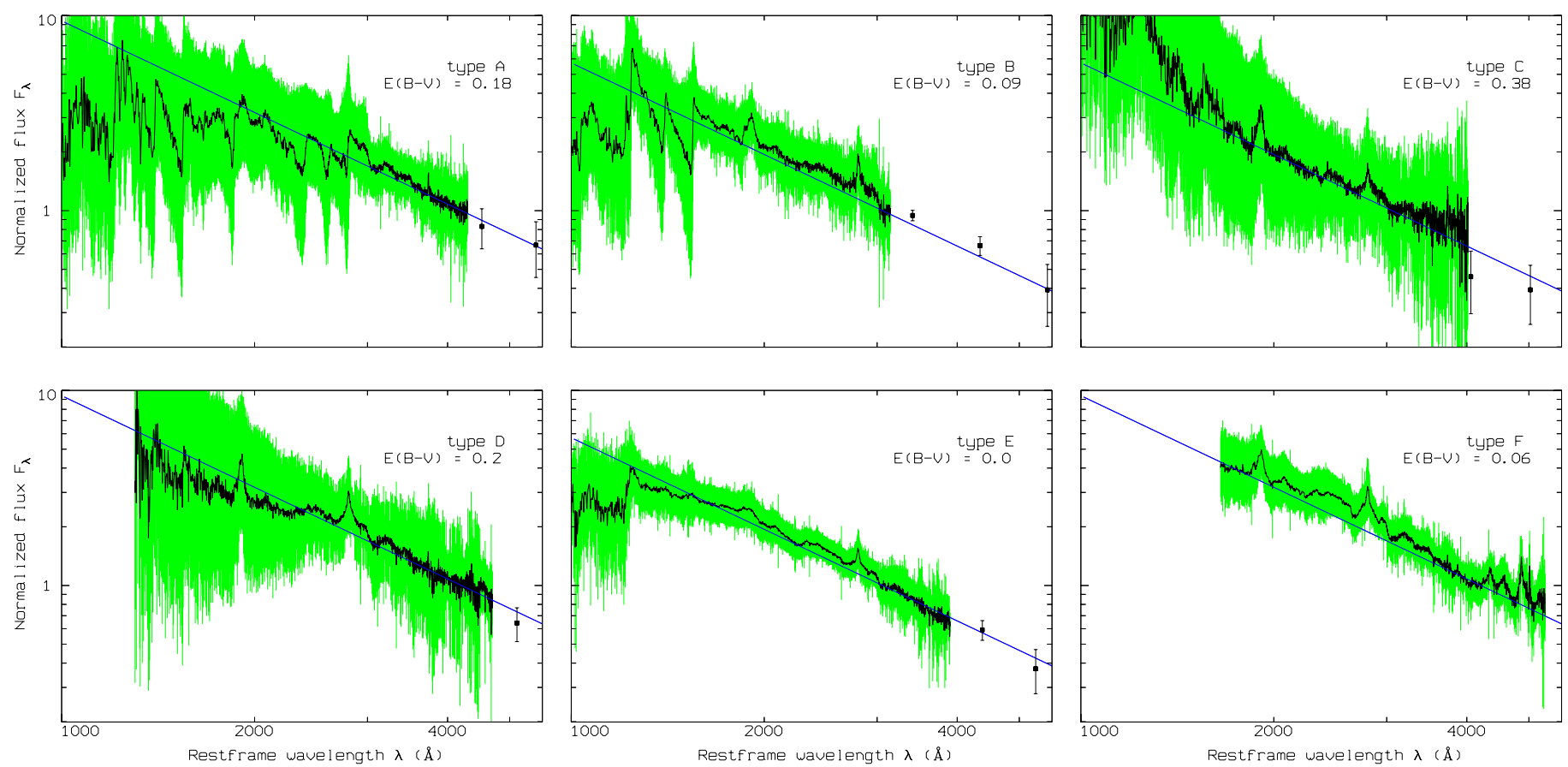

Fig. 9. Geometric mean composite spectra corrected for intrinsic reddening. The SDSS spectra were combined with $J H K$ fluxes form $2 \mathrm{MASS}$ (squares with error bars). For comparison, the power-law continuum fit to the geometric mean SDSS composite from Vanden Berk et al. (2001) is shown.

the flux density of $\boldsymbol{C}_{1}$ at $\lambda=3030-3090 \AA$ and the normalised spectra were co-added together with the normalized spectra from the previous step to create a second composite $\boldsymbol{C}_{2}$. In the same way, the continuum window at $\lambda=2200-2230 \AA$ was used to extend the composite $\boldsymbol{C}_{3}$ to $z=3$. To include quasars at higher redshift, we introduced another pseudo-continuum window at $\lambda=1650-1700 \AA$ where the spectra with $z>3$ were scaled to the flux density of $\boldsymbol{C}_{3}$.
Combining the normalised spectra to a composite requires choosing between mean or median and arithmetic or geometric averaging. Following Vanden Berk et al. (2001), we used combining techniques to create $(a)$ the arithmetic median, which preserves the relative fluxes along the spectra, and (b) the geometric mean spectrum, which preserves the global shape of a power-law continuum. The arithmetic median spectrum was computed in the same way as in our previous study 
(Meusinger et al. 2011): all normalised (restframe) spectra $\boldsymbol{S}_{i}$ of a given type were inserted into a $2 \mathrm{D}$ image, one spectrum per row with $1 \AA$ binning on the horizontal axis. The arithmetic median was then computed by averaging over each column using the procedure average/image from the ESO-MIDAS package ${ }^{11}$ with the median average option. A similar approach to the previous section was used for the geometric mean, where however the 2D image was build from the $\log \boldsymbol{S}_{i}$ instead of $\boldsymbol{S}_{i}$ to compute the mean value $\langle\log S\rangle_{i}$. The geometric mean spectrum $S_{\mathrm{gm}}$ and the geometric standard deviation $\sigma_{\mathrm{g}}$ are given by

$\boldsymbol{S}_{\mathrm{gm}}=\left[\prod_{i=1}^{N} \boldsymbol{S}_{i}\right]^{1 / N}=10^{\left\langle\log \boldsymbol{S}_{i}\right\rangle}, \quad \sigma_{\mathrm{g}}=10^{\sqrt{\left\langle\left[\log \left(\boldsymbol{S}_{i} / \boldsymbol{S}_{\mathrm{gm}}\right)\right]^{2}\right\rangle}}$.

The resulting spectra are shown on a log-log scale in Fig. 8 for the arithmetic median and in Fig. 9 for the geometric mean (extinction-corrected, see below), respectively, except for type $\mathrm{M}$ where a composite makes no sense as this subsample is too small and very heterogeneous. The spectra of different types have different normalisations owing to their different wavelength coverages. The $1 \sigma$ variation is shown by the shaded area. The variation in the flux density across the spectrum is expected to reflect the spectrum-to-spectrum differences caused by differences in the continuum shapes and in the properties of the emission and/or absorption lines (see Vanden Berk et al. 2001).

The average rest-frame spectra differ significantly from type to type, as well as from the Vanden Berk composite of "normal" SDSS quasars. The defining spectral features are clearly indicated in the median spectra. The median spectrum of the unusual BAL quasars of type A is remarkably dominated by the typical absorption troughs of FeLoBAL quasars (see Hall et al. 2002; their Table 1), of which the strongest are indicated by the dashed vertical lines: Ly $\alpha, \mathrm{N} v \lambda 1334, \mathrm{C}$ II $\lambda 1334$, Si Iv $\lambda 1398$, C IV $\lambda 1550, \mathrm{Al}_{\text {III }} \lambda 1860$, Fe II $\lambda 2400$, Fe II $\lambda 2600$, Fe II $\lambda 2750$, and $\mathrm{Mg}$ II $\lambda 2800^{12}$. On the other hand, the spectrum-to-spectrum variations for type A are huge. This reflects the particularly high degree of diversity among these spectra. As expected, the dominating spectral features in the median spectrum for type B are the absorption troughs from the high ionisation lines $\mathrm{O}$ IV $\lambda 1033$, Ly $\alpha, N_{\text {IV }} \lambda 1240$, Si IV $\lambda 1398$, and C IV $\lambda 1550$. There are no stringent indications for BALs in the median spectra of the other types, though these features are present in the spectra of some members of these types (Sect. 4.3).

\subsection{Intrinsic reddening and corrected absolute magnitudes}

The general trend of BAL quasars to show stronger UV reddening than non-BAL quasars and of LoBAL quasars to be even redder (see Sect. 5.4) is clearly indicated by the type composites in Fig. 8. The type F composite indicates significant reddening shortwards of $\mathrm{Mg}$ II. For type $\mathrm{E}$, we found that quasars with $z \lessgtr 2$ tend to have steeper continua than normal quasars (e.g., Fig. 5), but that the spectra are slightly shallower at higher $z$. This yields an average spectrum for type $\mathrm{E}$ with a continuum that reasonably fits the SDSS composite of normal quasars. The quasars of the types $\mathrm{C}$ and $\mathrm{D}$ are by definition redder than normal quasars.

We assumed that the red continua are due to reddening by dust related to the quasar and/or the host galaxy and estimated

\footnotetext{
11 http://www.eso.org/sci/software/esomidas

12 Most of these lines are actually blends of two or several lines. However, in many cases throughout this paper it is adequate to treat these blends as single lines.
}

the mean intrinsic reddening $\left\langle E_{B-V}^{(\mathrm{int})}\right\rangle$ of each type from its geometric mean spectrum

$\log S_{\mathrm{gm}, 0}=\log \boldsymbol{S}_{\mathrm{gm}}+0.4\left\langle E_{B-V}^{\text {(int) }}\right\rangle\left[R_{V}+Q_{\lambda}\right]$,

where $S_{\mathrm{gm}}$ is the observed geometric mean from Eq. (8) and $S_{\mathrm{gm}, 0}$ is the emitted geometric mean spectrum before dust extinction. As has been shown in several previous studies (e.g., Reichard et al. 2003), SMC dust provides acceptable fits for the intrinsic reddening in quasars. Hence, we adopted the SMC extinction curve with $R_{V}=2.93$ and $Q(\lambda)=E_{\lambda-V} / E_{B-V}$ from Pei (1992). We assumed that $\boldsymbol{S}_{\mathrm{gm}, 0}$ can be identified with the geometric mean spectrum of usual quasars. Vanden Berk et al. (2001) found that a single power-law adequately fits the continuum between Ly $\alpha$ and $\mathrm{H} \beta$ with $\alpha_{v}=-0.44$. We used $\left\langle E_{B-V}^{(\mathrm{int})}\right\rangle$ in Eq. (9) as a free parameter to fit the continuum of the corrected spectrum of each type to the power-law with $\alpha_{v}=-0.44$ and identified the best-fit value with the mean reddening parameter of this type.

This approach is hampered by the UV $(\lambda \lesssim 3000 \AA)$ continua being strongly affected by the unusual absorption and/or emission features, perhaps with the exception of type E. On the other side, the composite spectra do not cover a wide wavelength interval longwards of $3000 \AA$, except for type F. To stretch the useful wavelength interval for the continuum fit, we combined the SDSS spectra with the fluxes derived from $J H K$ magnitudes given in the 2MASS catalogue (Skrutzki et al. 2006). The percentage of quasars with 2 MASS magnitudes varies between $45 \%$ for type B and $70 \%$ for type A with a mean value of $58 \%$. For each object identified in 2MASS, the JHK fluxes were normalised with the same normalisation factors used for the corresponding spectrum. The fluxes derived from the 2MASS magnitudes generally agree with the extrapolation of the composite spectra towards longer wavelengths.

The extinction-corrected geometric mean spectra are shown in Fig. 9. The mean reddening parameters are given in Table 4. The values are larger than those found in previous studies (e.g., $E_{B-V}=0.08$ (0.02) for LoBALs (HiBALs) according to Reichard et al. 2003), which is most likely due to differences between the quasar samples. The contributions from host galaxies in the optical are obviously suppressed by the strong fluxes from the bright quasars.

As can be seen in Fig. 9, the rest-frame optical colours of the unusual quasar types are essentially the same as for the SDSS quasar composite (for a similar result see, e.g., Zhang et al. 2010), while they tend to be redder in the UV. In particular, the UV flux of the unusual BAL quasars of type A is substantially lower than the normal BAL quasars of type B. This is likely caused by quasars for which the UV flux is strongly suppressed by BAL troughs and/or overlapping troughs (Sect. 6.1). Owing to these features, the fit to the type A composite is particularly uncertain. Types B and F are well-fitted by the SDSS continuum. No reasonable fit can be achieved for type $D$, where the slope of the dereddened continuum becomes shallower shortwards of $\mathrm{Mg}$ II. Since a substantial fraction of red quasars also show BALs (Sect. 4.3), the combined effect of broad-line absorption may have some influence on the average continuum, but there are no strong indications of BALs in the type D composite spectrum. An extinction curve steeper than the SMC curve would be another explanation. Finally, the type E geometric mean spectrum fits the SDSS composite at longer wavelengths without dereddening but flattens approximately below the C IV line. This may be an indication for differences between the weak-line quasars of high and low redshift. 


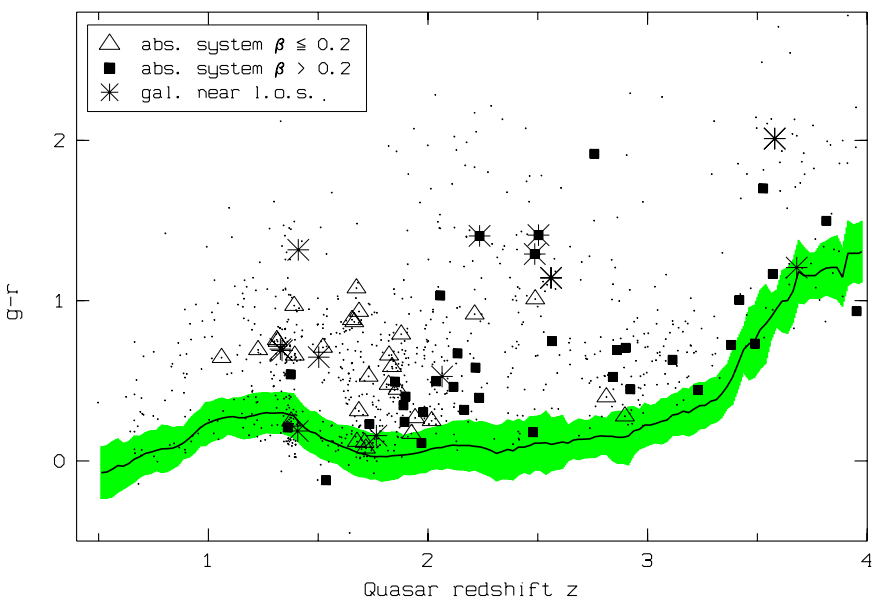

Fig. 10. Colour indices $g-r$ (foreground extinction-corrected) as function of redshift for all types (dots). Other symbols: quasars with indications of foreground systems.

In the next step, we used the mean reddening parameters from Table 4 to estimate the absolute magnitudes statistically corrected for intrinsic dust extinction adopting the approximation

$\left\langle M_{i}^{(\mathrm{cor})}\right\rangle \approx\left\langle M_{i}\right\rangle-\left\langle E_{B-V}^{(\mathrm{int})}\right\rangle \cdot\left[R_{V}-\left\langle Q\left(\lambda_{\mathrm{i}, \mathrm{eff}}^{(\mathrm{rf})}\right)\right\rangle\right]$,

where the angular brackets symbolise the ensemble average and $\lambda_{i, \mathrm{eff}}^{(\mathrm{rf})}=\lambda_{i, \mathrm{eff}} /(1+z)$ is the rest-frame wavelength corresponding to the effective wavelength of the $i$ band with $\lambda_{\mathrm{i} \text {,eff }}=7461 \AA$ (Schneider et al. 2010). We again adopted the SMC extinction curve from Pei (1992). As can be seen from the results listed in Table 4, this correction is significant. The one-sided KS (Sect. 5.2) applied to the corrected $M_{i}^{\text {(cor) }}$ inferred that all six quasar types $\mathrm{A}$ to $\mathrm{F}$ are on average more luminous then the comparison samples of normal quasars. The finding of the weaker C IV emission of BAL quasars compared to that of nonBAL quasars (Gibson et al. 2009) thus appears as a manifestation of the Baldwin-effect (Baldwin 1977; Osmer et al. 1994) relation between the $\mathrm{C}$ IV emission line strength and the UV luminosity.

\subsection{Intervening absorbers}

Finally, we checked by eye the spectra of all quasars for narrow absorption lines distinct from the quasar lines and at lower $z$ than the quasar. One of the easiest species to detect is the $\mathrm{Mg}$ II $\lambda \lambda 2796,2804$ doublet, which is frequently in combination with Fe II $\lambda \lambda 2587,2600$, Fe II $\lambda \lambda 2374,2382$, Fe II $\lambda 2344$, and Mg I 22853 . While the origin of the absorbing gas remains unclear, a direct association with galaxies is suggested by several studies (e.g., Bahcall \& Spitzer 1969; Steidel et al. 1997; Bowen $\&$ Chelouche 2011; Kacprzak et al. 2011). Dust embedded in foreground absorber systems along the line of sight is expected to absorb and scatter the UV photons from the quasar and redden the quasar spectrum. Detection of the quasar reddening related to absorber systems has been reported e.g. by York et al. (2006) and Ménard et al. (2008) for Mg II, by Wild et al. (2006) for Ca II, and by Vladilo et al. (2008) for damped Ly $\alpha$ absorber systems. While the extinction curves of the absorbers are generally considered to be consistent with that of the SMC, there are probably also cases of Milky Way-like dust extinction that can strongly affect the quasar spectrum in a narrower wavelength interval owing to the broad extinction bump around $2175 \AA$ (Jiang et al. 2011).
Table 6. Unusual quasars with structures close to the sightline on the SDSS images.

\begin{tabular}{lccccc}
\hline \hline SDSS J & $z$ & $T$ & Object $^{a}$ & $\mathrm{AL}^{b}$ & Ref. \\
\hline $024230.65-000029.7$ & 2.486 & $\mathrm{~B}$ & NGC 1068 & no & \\
$075518.31+245432.3$ & 1.335 & $\mathrm{C}$ & gal & no & \\
$081959.80+535624.2$ & 2.235 & $\mathrm{C}$ & gal & yes & 1 \\
$090334.94+502819.3$ & 3.579 & $\mathrm{C}$ & gal group & yes & 2 \\
$103410.26+344200.7$ & 2.503 & $\mathrm{~B}$ & $?$ & yes & \\
$103443.66+411120.4$ & 2.064 & $\mathrm{~F}$ & gal & no & \\
$104513.86+171952.2$ & 3.679 & $\mathrm{E}$ & gal & $?$ & \\
$112851.83+062315.3$ & 1.503 & $\mathrm{~A}$ & gal & $?$ & \\
$135048.67+433406.2$ & 1.765 & $\mathrm{E}$ & gal & no & \\
$143445.95+422516.7$ & 1.407 & $\mathrm{~F}$ & gal & no & \\
$144002.24+371058.5$ & 1.409 & $\mathrm{~A}$ & gal & no & \\
$151150.23+140506.4$ & 2.156 & $\mathrm{E}$ & gal group & no & \\
$164847.85+374006.3$ & 1.328 & $\mathrm{C}$ & gal & no & \\
\hline
\end{tabular}

Notes. ${ }^{(a)}$ Foreground object: gal = galaxy, ? = unclear; ${ }^{(b)}$ absorption line system in SDSS spectrum.

References. (1) Inada et al. (2010); (2) Johnston et al. (2003).

We identified 76 absorber systems in 66 quasar spectra with a mean absorber redshift $\left\langle z_{\text {abs }}\right\rangle=1.44 \pm 0.45$. Among them are 8 quasars with two and one quasar (SDSS J082747.14+425241.1) with three different absorber systems. The redshift differences between the absorber and the quasar, expressed by the velocity parameter $\beta=v / c=\left(R^{2}-\right.$ $1) /\left(R^{2}+1\right)$, where $R=\left(1+z_{\mathrm{q}}\right) /\left(1+z_{\mathrm{abs}}\right)$, covers the range $\beta=$ 0.02 to 0.72 (mean value 0.28 ). The fraction of quasars with registered absorption systems strongly depends on the spectral type. It is lowest for type A (3\%) and highest for type E (13\%). This is almost certainly a selection bias related to the intrinsic complexity of the type A spectra. For any given spectral type, on the other hand, quasars with or without these narrow-line absorber systems have on average the same peculiarity parameters $\chi^{2}$.

Figure 10 shows the colour index $g-r$ (corrected for Galactic extinction) versus $z$ for all unusual quasars (as in Fig. 7), where those showing indications of foreground galaxies are marked by symbols. Since absorbers with small $\beta$ may be associated with the quasar, we split the sample into two groups of 29 quasars with $\beta_{\max } \leq 0.2$ and 37 with $\beta_{\max }>0.2$. As outflows from quasars usually have $\beta<0.2$ (Foltz et al. 1983), the latter group (with $\langle\beta\rangle=0.41 \pm 0.14$ ) is expected to represent a clean sample of quasars with spectroscopically identified foreground galaxies. The majority of our quasars with foreground galaxies are redder than the normal quasars from the QCDR7. Hence, we cannot exclude that a fraction of the red quasars (types 3 and 4) were selected as unusual owing to properties related to the foreground rather than the quasar itself (as is indeed the case for the two gravitationally lensed quasars SDSS J081959.80+535624.2 and SDSS J090334.94+502819.3). However, we did not find any significant differences between the reddening properties of these quasars in our sample showing evidence of foreground galaxies close to the line of sight and those which do not.

In Appendix A, we present the results of a simple search for extended structures close to the line of sight on direct images.

\section{Very peculiar quasar spectra}

We did not identify any fundamentally new type of unusual quasar spectra. However, our selection substantially enlarges the sample of known unusual quasars and thus enables statistical studies of the various peculiarity types and searches for relations 
between them. Representative examples of our 7 types were shown in Fig. 5. The detailed investigation of individual spectra is beyond the scope of this paper. However, it is worth commenting on a few very peculiar and interesting spectra, including another three mysterious objects and another dozen possibly related quasars.

\subsection{Unusual BALs and strong Fe emission}

A selection of SDSS quasars with particularly unusual spectra caused by extreme BAL features or/and strong iron emission is presented in Fig. 11. Each panel shows the normalised flux $F_{\lambda}(\lambda)$ over the observed wavelength interval $3900 \AA$ to $9000 \AA$, the object name is given at the top of the panel. Here we briefly discuss these 12 spectra from top to bottom and left to right.

The first three panels show quasars with strong iron emission. In the first panel, we present SDSS J100237.22+ $270056.5^{13}$ which exhibits emission from the multiplets Fe II $\lambda 1785$ (UV67, UV191), Fe III $\lambda 1926$ (UV34), Fe III $\lambda 2070$ (UV48), Fe II $\lambda 2400$ (UV2), Fe II $\lambda 2600$ (UV1), and Fe II $\lambda 2750$ (UV62,UV63). The positions of these lines, as well as the positions of the typical quasar emission lines (Si Iv $\lambda 1400$, C IV $\lambda \lambda 1542.2,1550.8, \mathrm{Al}$ III $\lambda \lambda 1854.7,1862.8, \mathrm{C}$ III] $\lambda$ 1908.7, Mg II $\lambda \lambda 2796.3,2803.5)$, are marked by the vertical solid lines. There is a high-velocity outflow at $z_{\mathrm{abs}}=1.724$ seen in $\mathrm{C}$ IV, $\mathrm{Al}$ II, $\mathrm{Al}$ III, and $\mathrm{Mg}$ II. The absorption line redward of $\mathrm{C}_{\mathrm{IV}}$, close to $\mathrm{He}$ II $\lambda 1640$, is probably a narrow intervening system. SDSS J100237.2+270057 is one of the most luminous quasars in our sample with $M_{i}=-28.94$.

The spectrum in panel (b) shows a quasar (SDSS J092211.56+365120.2) where the emission from Fe II UV1 and UV2 and Fe III UV48 is stronger than the (weak) broad emission lines $\mathrm{C}_{\mathrm{IV}}$ and $\mathrm{Mg}$ II. Strong iron emission is known to be correlated with the occurrence of BAL troughs (Boroson \& Meyers 1992; Zhang et al. 2010), as can be seen in many of our spectra of types A or F (Sect.4.3). A nice example is SDSS J094225.42+565613.0 in panel (c). The positions of absorption troughs at $z_{\text {abs }}=0.735$ are marked by the dashed lines. If we adopt the systemic redshift of SDSS J094225.42+565613.0 from the (weak) [O II] $\lambda 3728$ emission line, the $\mathrm{Mg}$ II emission line is either blueshifted or absorbed at the red side. In Table 3 , the remark "lst?" is made, indicating possible but not certain longwards-of-systemic absorption (see Hall et al. 2002). The Mg II emission of SDSS J094225.42+565613.0 may instead be affected in this case by broad Fe II absorption on its red side.

As a consequence of the overlapping Fe II troughs, the continuum of SDSS J094225.42+565613.0 is depressed shortwards of $\mathrm{Mg}$ II down to the lowest observed wavelengths. Interestingly, the Fe II UV2 trough appears to be deeper than UV1. As outlined by Hall et al. (2002), the only way of explaining an increase in the absorption strength when troughs overlap is spatially distinct velocity-dependent partial covering (sdvdpc) of the continuum source, provided that the troughs are saturated (as is usually the case even when the absorption is not black). Hall et al. emphasise that these objects "may not be all that rare, just difficult to recognise", though the only quasar known to them to definitely exhibit sdvdpc was FBQS $1408+3054$. From the inspection of our sample, we found at least another eight candidates probably showing that effect (Table 7).

In the second row of Fig. 11, we give three examples of LoBAL quasars with possible strong Fe III absorption.

\footnotetext{
13 A similar object (not shown here) is SDSS J124244.36+624659.1.
}

Table 7. Candidates for spatially distinct velocity-dependent partial covering (sdvdpc) of the continuum source by Fe II UV troughs.

\begin{tabular}{lccc}
\hline \hline SDSS J & $T$ & $z$ & Remark \\
\hline $015151.58-093215.3$ & A & 1.413 & ot(sdvdpc) \\
$082350.53+244653.1$ & C & 1.766 & ot(sdvdpc)? \\
$094225.42+565613.0$ & A & 0.831 & ot(sdvdpc?, lst?); sFe(opt) \\
$101912.84+410807.4$ & A & 2.450 & nt(sdvdpc); Fe III BAL? \\
$135246.37+423923.5$ & A & 2.000 & ot(sdvdpc) \\
$142010.28+604722.3$ & A & 1.349 & ot(sdvdpc) \\
$150848.80+605551.9$ & A & 1.525 & ot(sdvdpc?) \\
$162527.73+093332.8$ & A & 1.413 & ot(sdvdpc) \\
\hline
\end{tabular}

Notes. Remarks see Table 3.

SDSS J021102.33-081007.4 resembles the two possible Fe III BAL quasars SDSS J014905.28-011404.9 and SDSS J081024.75+480615.4 from Hall et al. (2002). It appears to have Fe III UV34, UV48 troughs (which probably overlap), in addition to troughs from Si IV, C IV, Mg II, and weak Fe II UV1, UV2 absorption. Even more pronounced are the iron absorption troughs (plus troughs from other elements) seen in SDSS J101912.84+410807.4. For both spectra, and also for all following quasars shown in Fig. 11, it seems impossible to clearly identify any emission line, thus only absorption redshifts can be given. The BAL quasars can show, of course, two or more absorption systems at different $z_{\text {abs }}$. The values of $z_{\text {abs }}$ given in Fig. 11 usually refer to the centres of the strongest system.

The third row shows another three examples for FeLoBAL quasars with many narrow troughs, similar to the three high- $z$ quasars of this category discussed by Hall et al. (2002). Although the spectra appear to be very complex, a large number of absorption features can be identified. Only a selection of the strongest troughs is labelled here. We again did not try to estimate the emission redshift since emission lines can be mimicked by overlapping absorption troughs that make detailed line identification quite difficult. As for the previously known objects of this type, no absorption trough reaches zero flux, indicating that there is a partial coverage of the continuum source by the absorbers and/or a contribution from scattered light (see Hall et al. 2002).

The SDSS has discovered several FeLoBAL quasars with sharply declining flux near $\mathrm{Mg}$ II, which is interpreted as being caused by overlapping iron troughs (Hall et al. 2002). The bottom row of Fig. 11 exhibits three examples where the absorption strength appears to increase from left to right. Here, the redshifts refer to the red edges of the absorption troughs. Only the longest wavelength lines are marked for the Fe multiplets. SDSS J162527.73+093332.8 displays both many narrow troughs at $\lambda \lesssim 7200 \AA$ (observed) and overlapping Fe II UV1 and UV2 at longer wavelengths (Fe II UV2 is deeper than UV1, see above).

SDSS J173049.10+585059.5 was already described by Hall et al. (2002). With the exception of two narrow wavelength intervals around $\left.C_{\mathrm{III}}\right] \lambda 1908.7$, there is almost no flux shortwards of the onset of Fe II UV2 at $7000 \AA$ (observed) down to the shortest wavelengths. A similar spectrum, but even more extreme, is that of SDSS J094317.59+541705.1 at the right-hand side. The flux drops rapidly at $\sim 9000 \AA$ (observed) towards shorter wavelengths and is below the detection threshold in the SDSS $g$ and $\mathrm{u}$ bands, i.e. shortwards of the $\mathrm{C}$ IV line. The object is detected in 2MASS with $K=14.25$. The 2MASS colours correspond to a slowly decreasing flux with increasing wavelengths 

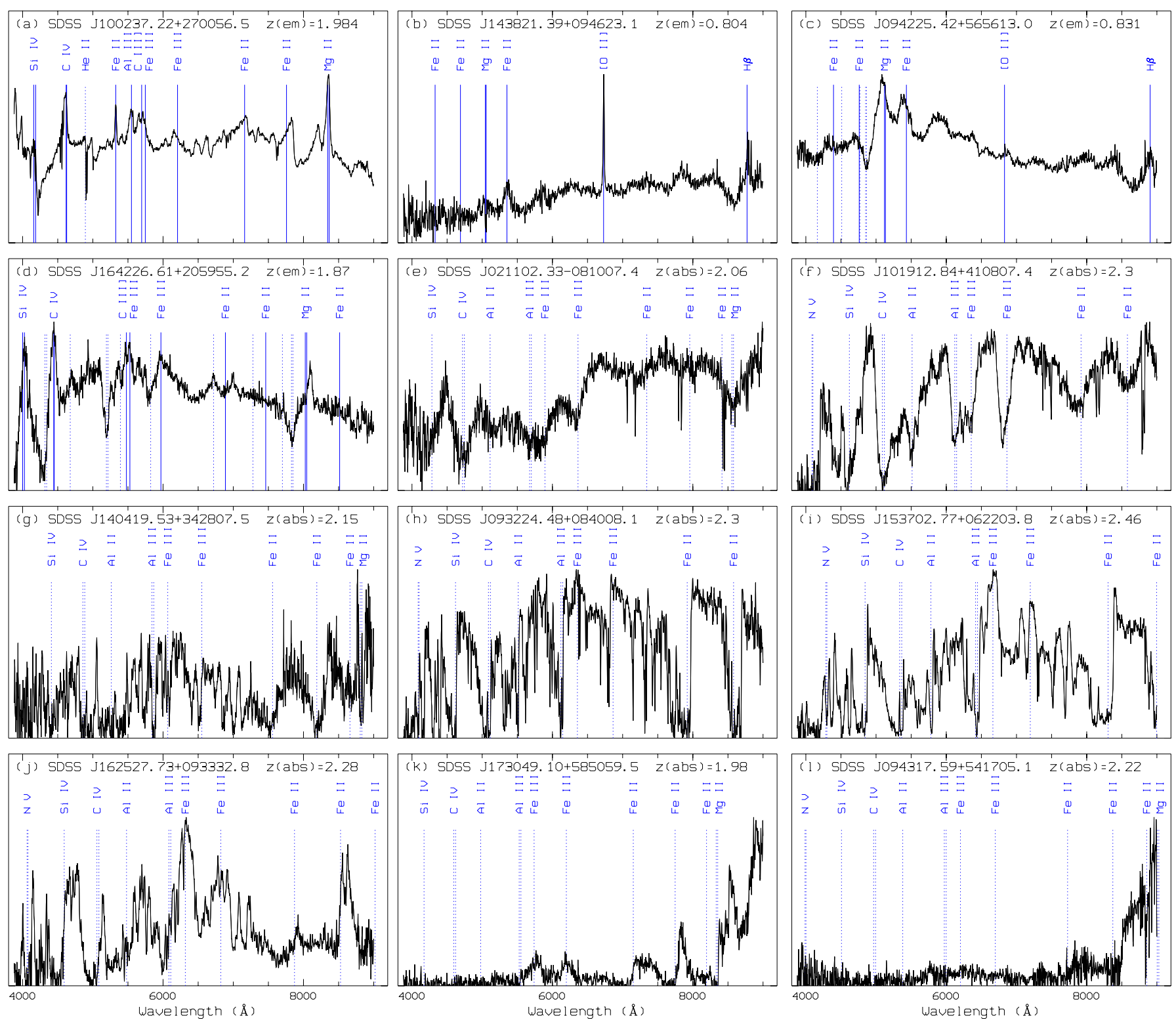

Fig. 11. Examples of particularly unusual SDSS quasars: strong UV iron emission (top), Fe III absorption (second row), and BAL quasars with both many troughs (third row), and with overlapping troughs (bottom).

from 1.2 to $2.2 \mu \mathrm{m}$. At first glance, the SDSS spectrum suggests a high redshift quasar at $z>6$. However, a higher-quality Keck spectrum presented by Urrutia et al. (2009) clearly indicates the $\mathrm{Mg}$ II emission line, along with the typical absorption features of a FeLoBAL at $z=2.224$.

Given that SDSS J162527.73+093332.8 is more luminous than the other two overlapping trough quasars SDSS J173049.10+585059.5 and SDSS J094317.59+541705.1 $\left(M_{i}=-26.27\right.$ compared to -24.65 and -24.75$)$, all three spectra are most likely very similar. The main difference is a slightly higher redshift of SDSS J162527.73+093332.8, hence the $3000 \AA$ jump is shifted out of the spectral window. The very red optical-to-near infrared colours of SDSS J162527.73+093332.8 (which is one of the reddest SDSS quasars; $r-K=7.17$ ) indicate that its flux must strongly increase longwards of the red edge of the SDSS spectrum. Lower-luminosity versions of these quasars at $z \gtrsim 2.3$ are strongly biased against being included in optical magnitude-limited samples.

\subsection{Mysterious and possibly related objects}

Hall et al. (2002) discussed at length two objects discovered by the SDSS with spectra classified by these authors as "mysterious": SDSS J010540.75-003313.9 and SDSS J220445.27+003141.8 (Fig. 12b,c). The most prominent spectral features are: (a) a lack of substantial typical quasar emission lines (except broad Fe II emission and [O II] $\lambda 3730$ in the case of SDSS J010540.75-003313.9); (b) a blue continuum longwards of $\sim 3200 \AA$; (c) a dip at $\sim 3000 \AA$; $(d)$ a continuum drop-off shortwards of $\mathrm{Mg}$ II $\lambda 2800$ which appears too steep to be caused by dust reddening; and $(e)$ no obvious BAL troughs. Both objects show associated Mg II absorption lines, are unresolved FIRST radio sources, and are much more luminous than any galaxy. Hall et al. discussed various viable explanations for these spectra and concluded that none of them are particularly satisfactory. These strange objects may be very unusual BAL quasars with partial covering of different regions of the 
Table 8. Mysterious objects and possibly related quasars.

\begin{tabular}{|c|c|c|c|c|c|c|c|c|c|c|c|}
\hline Name & $\begin{array}{l}\text { Panel in } \\
\text { Fig. } 12\end{array}$ & $z$ & Ref. & $T$ & $\begin{array}{c}M_{i} \\
(\mathrm{mag})\end{array}$ & $R_{i}$ & $\begin{array}{c}E_{B-V}^{\text {(intr) }} \\
\text { (mag) }\end{array}$ & $\begin{array}{c}\mu_{\alpha} \cos \delta \\
(\mathrm{mas} / \mathrm{yr})\end{array}$ & $\begin{array}{c}\mu_{\delta} \\
\text { (mas/yr) }\end{array}$ & $N_{\mathrm{e}}$ & $\mathrm{pm}$ \\
\hline \multicolumn{12}{|l|}{ (a) Mysterious objects: } \\
\hline SDSS J010540.75-003313.9 ${ }^{a}$ & (c) & $1.179^{b}$ & 1 & $\ldots$ & -26.49 & 1.19 & 0.18 & $8.1 \pm 2.1$ & $-4.1 \pm 2.3$ & 40 & $\mathrm{pmx} ?^{d}$ \\
\hline SDSS J085502.20+280219.6 & (f) & 1.511 & & $\mathrm{D}$ & -25.55 & & 0.15 & $-8.1 \pm 1.0$ & $1.5 \pm 2.0$ & 13 & pmx $?^{d}$ \\
\hline FBQS J105528.80+312411.3 & (a) & 0.497 & 1 & $\mathrm{~F}$ & -25.13 & 1.16 & 0.45 & $-3.1 \pm 2.7$ & $-2.2 \pm 3.5$ & 7 & no $\mathrm{pm}$ \\
\hline SDSS J130941.35+112540.1 $1^{a}$ & (h) & $1.362^{b}$ & 3 & $\ldots$ & -26.62 & 0.72 & 0.20 & $-2.2 \pm 5.2$ & $-1.2 \pm 2.6$ & 10 & no $\mathrm{pm}$ \\
\hline VPMS J134246.24+284027.5 & (i) & 1.300 & 4 & & -25.36 & 0.89 & 0.12 & $3.7 \pm 5.8$ & $-0.5 \pm 5.9$ & 22 & no $\mathrm{pm}$ \\
\hline SDSS J145045.56+461504.2 & (g) & 1.894 & 3 & $\mathrm{D}$ & -26.48 & 1.17 & 0.30 & $3.5 \pm 5.7$ & $1.8 \pm 1.8$ & 6 & no $\mathrm{pm}$ \\
\hline SDSS J160827.08+075811.5 & (e) & $1.182^{b}$ & & $\mathrm{D}$ & -27.57 & 1.18 & 0.18 & $1.5 \pm 1.2$ & $1.6 \pm 1.9$ & 11 & no $\mathrm{pm}$ \\
\hline SDSS J161836.09+153313.5 ${ }^{a}$ & (d) & $1.358^{b}$ & & & -26.02 & 1.22 & 0.10 & $1.4 \pm 2.1$ & $-9.0 \pm 2.1$ & 8 & pmy? ${ }^{d}$ \\
\hline SDSS J220445.27+003141.8 & (b) & 1.353 & 1 & $\mathrm{D}$ & -27.81 & 0.49 & 0.22 & $-1.6 \pm 1.3$ & $-1.5 \pm 1.6$ & 23 & no $\mathrm{pm}$ \\
\hline \multicolumn{12}{|l|}{ (b) Possibly related objects: } \\
\hline SDSS J073816.91+314437.0tablefootmarka & (q) & 2.01 & 2 & $\ldots$ & -26.80 & 0.80 & 0.13 & $-0.1 \pm 1.7$ & $-0.6 \pm 3.4$ & 11 & no $\mathrm{pm}$ \\
\hline SDSS J075437.85+422115.3 & (s) & 1.964 & & $\mathrm{C}$ & -26.17 & 1.29 & 0.27 & $31.4 \pm 6.2$ & $27.3 \pm 8.0$ & 12 & $\mathrm{pm} ?^{e}$ \\
\hline SDSS J091613.59+292106.2 & (1) & $1.143^{b}$ & & $\mathrm{D}$ & -25.64 & $\ldots$ & 0.18 & $-0.4 \pm 1.1$ & $-3.4 \pm 1.4$ & 13 & no pm \\
\hline SDSS J091940.97+064459.9 & $(\mathrm{r})$ & $1.351^{b}$ & & A & -26.02 & 2.05 & 0.27 & & $\ldots$ & $\ldots$ & $\ldots$ \\
\hline SDSS J101723.04+230322.1 & (o) & 1.794 & & A & -27.18 & 1.30 & 0.20 & $4.6 \pm 2.6$ & $0.8 \pm 6.8$ & 7 & no $\mathrm{pm}$ \\
\hline SDSS J110511.15+530806.5 & $(\mathrm{t})$ & 1.936 & & $\mathrm{D}$ & -26.63 & & 0.50 & $4.8 \pm 2.6$ & $-1.7 \pm 2.4$ & 6 & no $\mathrm{pm}$ \\
\hline SDSS J120337.91+153006.6 & (n) & $1.238^{b}$ & & $\mathrm{~A}$ & -26.62 & 0.55 & 0.12 & & & & $\ldots$ \\
\hline SDSS J134951.93+382334.1 & (j) & $1.094^{b}$ & & $\mathrm{~A}$ & -25.20 & 1.12 & 0.30 & $2.4 \pm 3.6$ & $-9.3 \pm 5.2$ & 7 & no $\mathrm{pm}$ \\
\hline SDSS J151627.40+305219.7 & (p) & 1.846 & & $\mathrm{D}$ & -27.79 & 0.43 & 0.10 & $-1.8 \pm 1.5$ & $0.5 \pm 2.0$ & 11 & no $\mathrm{pm}$ \\
\hline SDSS J152438.79+415543.0 & (m) & $1.227^{b}$ & & A & -26.00 & 1.01 & 0.22 & $-2.7 \pm 6.4$ & $-1.5 \pm 6.8$ & 14 & no $\mathrm{pm}$ \\
\hline SDSS J215950.30+124718.4 & (k) & 1.516 & & $\mathrm{D}$ & -26.39 & $\ldots{ }^{c}$ & 0.50 & $-11.5 \pm 2.3$ & $2.0 \pm 6.0$ & 9 & $\mathrm{pmx} ?^{d}$ \\
\hline
\end{tabular}

Notes. ${ }^{(a)}$ Not in Table 3; ${ }^{(b)}$ redshift based on [O II]; ${ }^{(c)}$ not in FIRST survey area; ${ }^{(d)}$ "pmx?" and "pmy?" indicate small ( $\sim 10$ mas/yr) proper motion components $\mu_{\alpha} \cos \delta$ or $\mu_{\delta}$ respectively. These results are formally significant but doubtful, as caused by probably erroneous POSS 1 positions. ${ }^{(e)}$ "pm?" indicates formally significant proper motions in both components that are likely caused by a faint object close to the line of sight.

References. (1) Hall et al. (2002); (2) Hall et al. (2004); (3) Plotkin et al. (2008); (4) Meusinger et al. (2005).

continuum source as a function of velocity, probably in combination with moderate reddening.

Regardless of the physical reason for these mysterious spectra, they almost certainly represent quasar types that are extremely rare in presently available samples. Hall et al. (2002) were aware of only two objects with probably similar spectra, the low $-z$ quasars FBQS $1503+2330(z=0.40)$ and FBQS 1055+3124 $(z=0.49)$ from the FIRST Bright Quasar Survey (White et al. 2000). In a search for BLLac objects, Plotkin et al. (2008) revealed another two FIRST sources with SDSS spectra similar to the two Hall et al. objects SDSS J130941.35+112540.1 and SDSS J145045.56+461504.2. Neither of these two new objects ultimately survived as BL Lac candidates during the manual inspection by these authors. The first similar object that was not targeted as a radio source was VPMS J134246.24+284027.5, which was discovered during a variability and (zero-) proper motion selection of quasar candidates (Meusinger et al. 2005). All seven of these objects have counterparts in the FIRST images with radio fluxes on the mJy level and moderate or small radio-loudness parameters.

Browsing through our sample (Table 3) yields another three comparable SDSS objects and about one dozen possibly related objects with spectral properties that are partly similar to those of the mysterious objects. In addition, we checked a subsample of the UNKNOWNS (SPEC_CLN=0) in the DR7. Only $\sim 10^{3}$ spectra of relatively high $\mathrm{S} / \mathrm{N}$ were clustered in one single SOM. The resulting icon map was used solely for selecting spectra that resemble those of the mysterious objects mentioned above. Two additional objects of this type were found: the mysterious object SDSS J010540.75-003313.9 from Hall et al. (2002) and SDSS J073816.91+314437.0, which was discussed by Hall et al. $(2004)^{14}$.

Table 8 lists 9 mysterious objects and another 11 objects that may represent links between the mysterious and other unconventional quasar types ${ }^{15}$. The following five objects from this list are not included in Table 3: VPMS J134246.24+284027.5 was not targeted by SDSS, SDSS J010540.75-003313.9 and SDSS J073816.91+314437.0 were not classified as quasars in the SDSS DR7, and for both SDSS J130941.35+112540.1 and SDSS J161836.09+153313.5 the (wrong) redshifts given in the DR7 $(z=4.39$ and 4.376 , respectively) exceed our upper selection limit of $z \leq 4.3$ (Sect. 4.1). Table 8 does not include FBQS $1503+2330$ because the SDSS spectrum does not cover much of the wavelength range shortwards of $\mathrm{Mg}$ II. The objects that are not part of our unusual quasar sample are flagged in the first column of Table 8. For clarity, the second column gives the panel in Figs. 12 to 14 in which we plot the spectrum.

For 11 quasars, the redshift could be obtained from emission lines. In 9 cases (marked in Table 8), the systemic redshifts were computed from the [O II] $\lambda 3728$ line, for the other two spectra from $\mathrm{Mg}$ II and $\left.\mathrm{C}_{\mathrm{III}}\right]$. The redshifts of the remaining 9 objects

14 Another highly peculiar object from this SOM is SDSS J164941.87+401455.9. This spectrum has a similar type to SDSS J033810.85+005617.6 from Hall et al. (2002).

15 Since the selection criteria are not sharply defined, a few more quasars may be found with more or less similar peculiar spectra, such as SDSS J110711.40+082331.2, SDSS J140800.43+345124.7, or SDSS J100353.68+515457.0. 
were identified with those of the highest- $z$ absorption features. For a few objects, e.g., VPMS J134246.24+284027.5, the redshift is fairly uncertain.

All objects with dubious spectra were checked for proper motions (pm) as described in Sect.4.2. The pm data for VPMS J134246.24+284027.5 were taken from Scholz et al. (1997). A significant non-zero pm was found for the object SDSS J020731.81+004941.6 (Fig. 3), which was rejected thereafter from the quasar list. The determined pm components $\mu_{\alpha} \cos \delta$ and $\mu_{\delta}$ are listed in Table 8 , along with the formal errors and the number of epochs $N_{\mathrm{e}}$. The column "pm?" contains our conclusion, which is not based on the formal error alone (see Sect. 4.2). For 13 objects (76\%), the results are clearly consistent with the conclusion that no significant pm is detected. For three objects, non-zero pm in one direction cannot be definitely excluded. A formally significant pm was measured for SDSS J075437.85+422115.3. This result is doubtful, however, because the measurements are likely affected by a faint source close to the sightline. For the three objects without pm data in Table 8, the extragalactic origin is clearly indicated by redshifted emission lines.

The partial spectra in the rest-frame wavelength interval $1350-3800 \AA$ are shown in Fig. 12. The spectra were arbitrarily normalised and slightly smoothed by a threepixel boxcar, with the exception of the noisy spectrum of SDSS J085502.20+280219.6 where a seven-pixel boxcar was applied. For comparison, the SDSS quasar composite spectrum is shown in the last panel (bottom right). The vertical bars above the spectra indicate the typical strong quasar emission lines Si IV $\left.\lambda \lambda 1394,1403, C_{\text {IV }} \lambda \lambda 1548,1551, C_{\text {III }}\right] \lambda 1909$, and $\mathrm{Mg}$ II $\lambda \lambda 2796,2804$. The dashed lines below the spectra mark the same absorption lines as in Fig. 11. If the redshift was obtained from absorption features, the lines above and below the spectrum refer to the same $z=z_{\text {abs }}$. Exceptions are SDSS J101723.04+230322.1 and SDSS J073816.91+314437.0, where the solid lines mark the highest- $z$ and the dashed ones the strongest absorption troughs.

The three new mysterious objects (Fig. 12d-f) are SDSS J160827.08+075811.5 $(z=1.18)$, SDSS J161836.09+ $153313.5(z=1.358)$, and SDSS J085502.20+280219.6 $(z=1.511)$. Both SDSS J160827.08+075811.5 and SDSS J161836.09+153313.5 are similar to the Hall et al. object SDSS J010540.75-003313.9. A remarkable difference between these two spectra and the SDSS J010540.75-003313.9 spectrum is the more abrupt transition between the blue part and the red part of the continuum. At first glance, it is tempting to ascribe the pronounced peak to the $\mathrm{Mg}$ II line. However, if we adopt the redshift from the [O II] line as the systemic redshift, the peak is clearly placed shortwards of $2800 \AA$ in either case. For SDSS J160827.08+075811.5, the redshift of the [O II] line agrees very well with that of an (associated?) absorption line system seen in $\mathrm{Mg}$ II, Mg I, and Fe II. Another new mysterious object is SDSS J085502.20+280219.6, which also resembles SDSS J010540.75-003313.9 but has a stronger depression at shorter wavelengths.

Similar to the mysterious objects in Figs. $12 \mathrm{a}-\mathrm{i}$, the spectra of SDSS J134951.93+382334.1 and SDSS J215950.30+ 124718.4 in panels $(\mathrm{j})$ and $(\mathrm{k})$, respectively, show a break in their continuum around the position of the $\mathrm{Mg}$ II line. The redshift of $z=1.516$ for SDSS J215950.30+124718.4 was derived from a clearly identified narrow $\mathrm{Mg}$ II absorption doublet at the position of the break. Both SDSS J134951.93+382334.1 and SDSS J215950.30+124718.4 are overlapping trough
LoBAL quasars (see bottom row of Fig. 11) and possibly mark a link between overlapping-trough objects and mysterious objects. (The dropoff at Mg II in SDSS J215950.30+124718.4 is more abrupt than in any of the latter objects.) From this point of view, the spectra in panels $(\mathrm{h})$ to $(\mathrm{k})$ seem to represent a sequence of overlapping-trough absorption from a low covering factor in VPMS J134246.24+284027.5 to the highest covering factor in SDSS J215950.30+124718.4.

The spectra in Fig. $12 \mathrm{~m}-\mathrm{O}$ show narrower BAL troughs with low covering factors. SDSS J151627.40+305219.7 (panel p) is very similar to SDSS J073816.91+314437.0 (panel q) from Hall et al. (2004). The high-velocity BAL in SDSS J101723.04+230322.1 (panel o) makes it plausible that high-velocity BALs contribute to the former two spectra.

Since intrinsic reddening seems to be present in all spectra in Fig. 12, we attempted to correct for it and compare the dereddened spectra with the typical quasar spectrum, following Hall et al. (2002). As in Sect.5.5, we adopted the SMC extinction curve at the quasar redshift with $E_{B-V}^{(\text {intr })}$ as a free parameter. For $z \lesssim 1.5$, the object spectrum was fitted to the SDSS composite spectrum at wavelengths $\lambda \gtrsim 3000 \AA$. For the higher- $z$ quasars, we simply tried to fit at the longest wavelengths what almost certainly amounts to an underestimated amount of reddening. We note that the method of deriving $E_{B-V}^{\text {(intr) }}$ is only approximate and the results are expected to be rather uncertain, at least for some objects. The mean value $\left\langle E_{B-V}^{\text {(intr) }}\right\rangle=0.27 \mathrm{mag}$ of the individually estimated reddening parameters of the 8 type $D$ quasars from Table 8 is somewhat larger than the ensemble average of 0.20 mag derived from the geometric mean composite spectrum of the type D quasars (Table 4 and Sect. 5.5). For the five type A quasars in Table 8 , we have $\left\langle E_{B-V}^{\text {(intr) }}\right\rangle=0.22 \mathrm{mag}$ compared to 0.18 mag for the whole sample.

The ratios of the thus dereddened spectra to the SDSS composite are shown in Fig. 13 in the same style as in Fig. 12. The individual intrinsic reddening parameters are listed in Table 8. A perfect fit would result in a horizontal line at the level of unity. All dereddened spectra, perhaps with the exception of SDSS J091940.97+064459.9 in panel (r), show a depression of the flux relative to the composite at short wavelengths. In most but not all cases, this sharp decline sets in at wavelengths around the $\mathrm{Mg}$ II line. This behaviour may be caused by very wide iron absorption troughs that overlap at substantially different velocities (Hall et al. 2002). This depression is also seen in the spectra in rows 4 and 5, where the presence of absorption troughs from Mg II and Fe II UV1 and UV2 (plus Si II and Fe III UV34, UV48 in the case of SDSS J120337.91+153006.6, panel $(n))^{16}$ is more obvious. The combination of reddening and partial-covering overlapping-troughs could perhaps explain such objects as SDSS J145045.56+461504.2 (panel g) and SDSS J010540.75-003313.9 (panel c), while narrower troughs could perhaps explain the spectra in panels $(\mathrm{m})$ to (o). As a remarkable exception, the unusual spectrum of SDSS J091940.97+064459.9 can be explained by the $2175 \AA$ absorption trough at the quasar redshift (at which narrow associated $\mathrm{Mg}$ II absorption is seen).

Hall et al. (2002) discussed, among others, the possibility that the strange continua could represent reddened versions of quasars with weak $\mathrm{Mg}$ II but strong broad Fe II emission. To check this idea for the quasars in Fig. 12, we repeated

16 SDSS J152438.79+415543.0 and SDSS J120337.91+153006.6 are another two sdvdpc-candidates with deeper Fe II UV2 than UV1 (Sect. 6.1). 
H. Meusinger et al.: Kohonen-selected unusual SDSS quasars
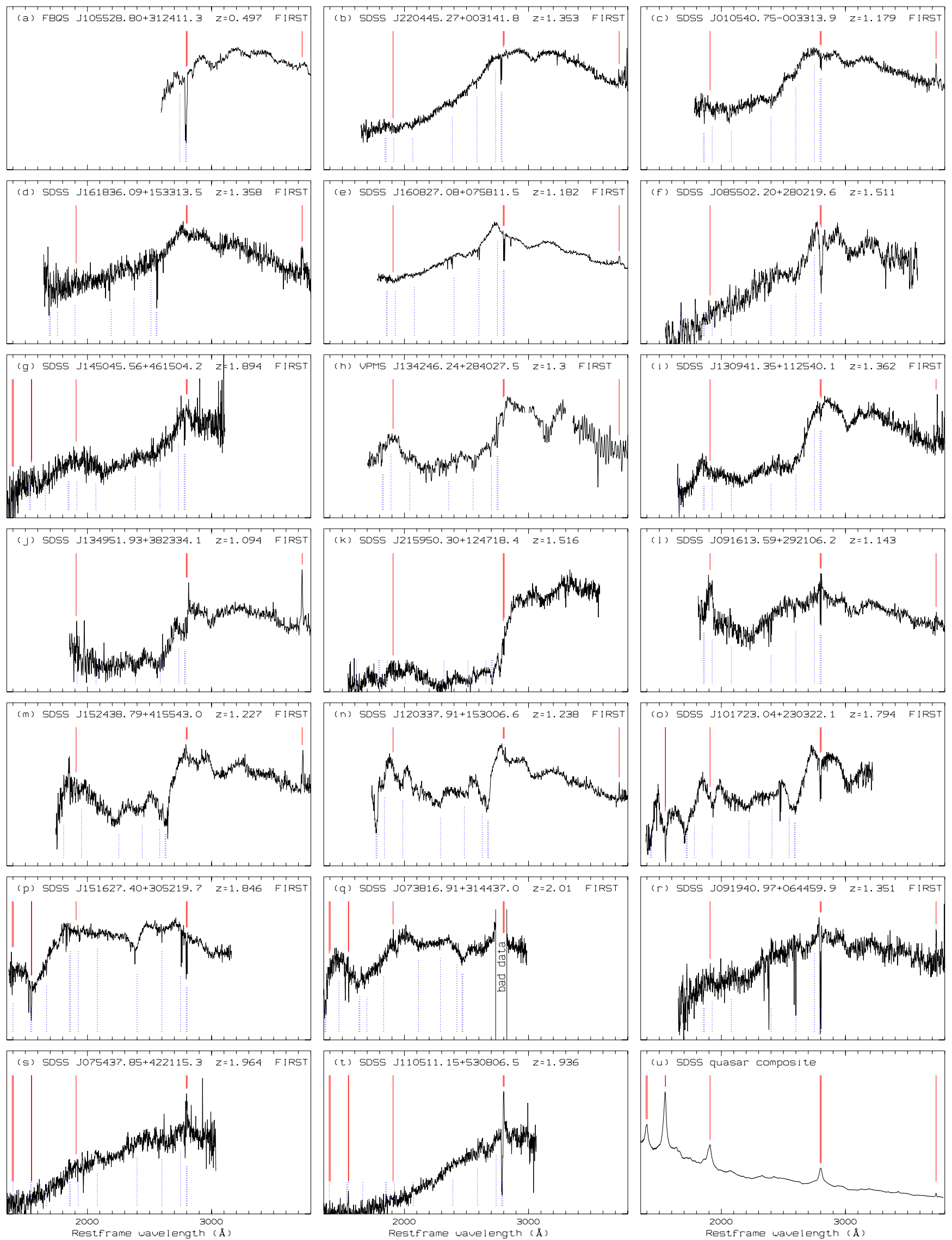

Fig. 12. Partial spectra of 9 mysterious objects and 11 possibly related quasars. Radio sources detected in FIRST are labelled. Vertical bars: positions of typical strong quasar emission (solid) and absorption (dashed) lines, respectively. 
A\&A 541, A77 (2012)
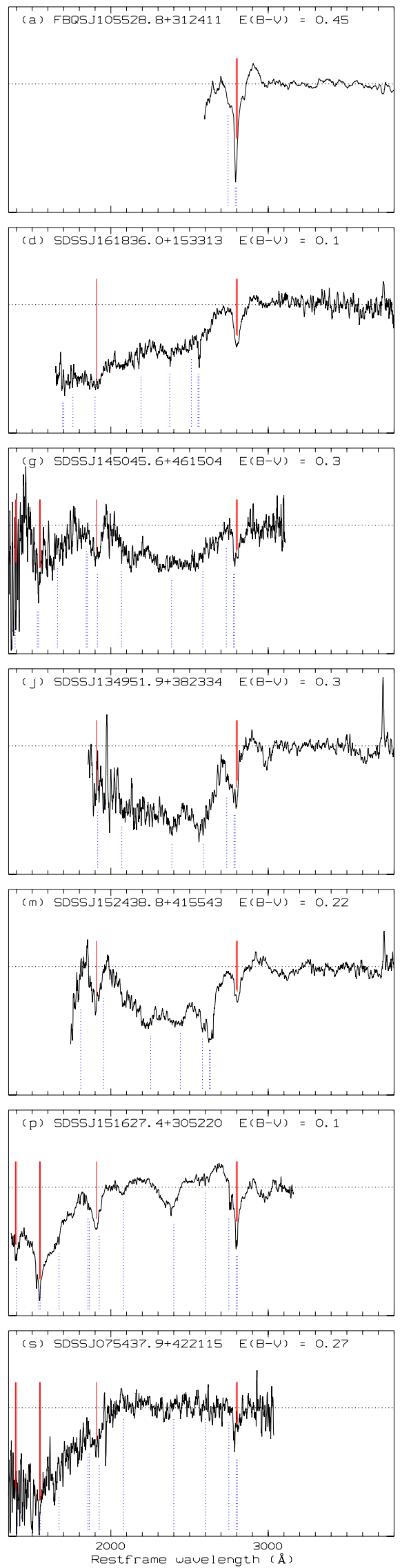
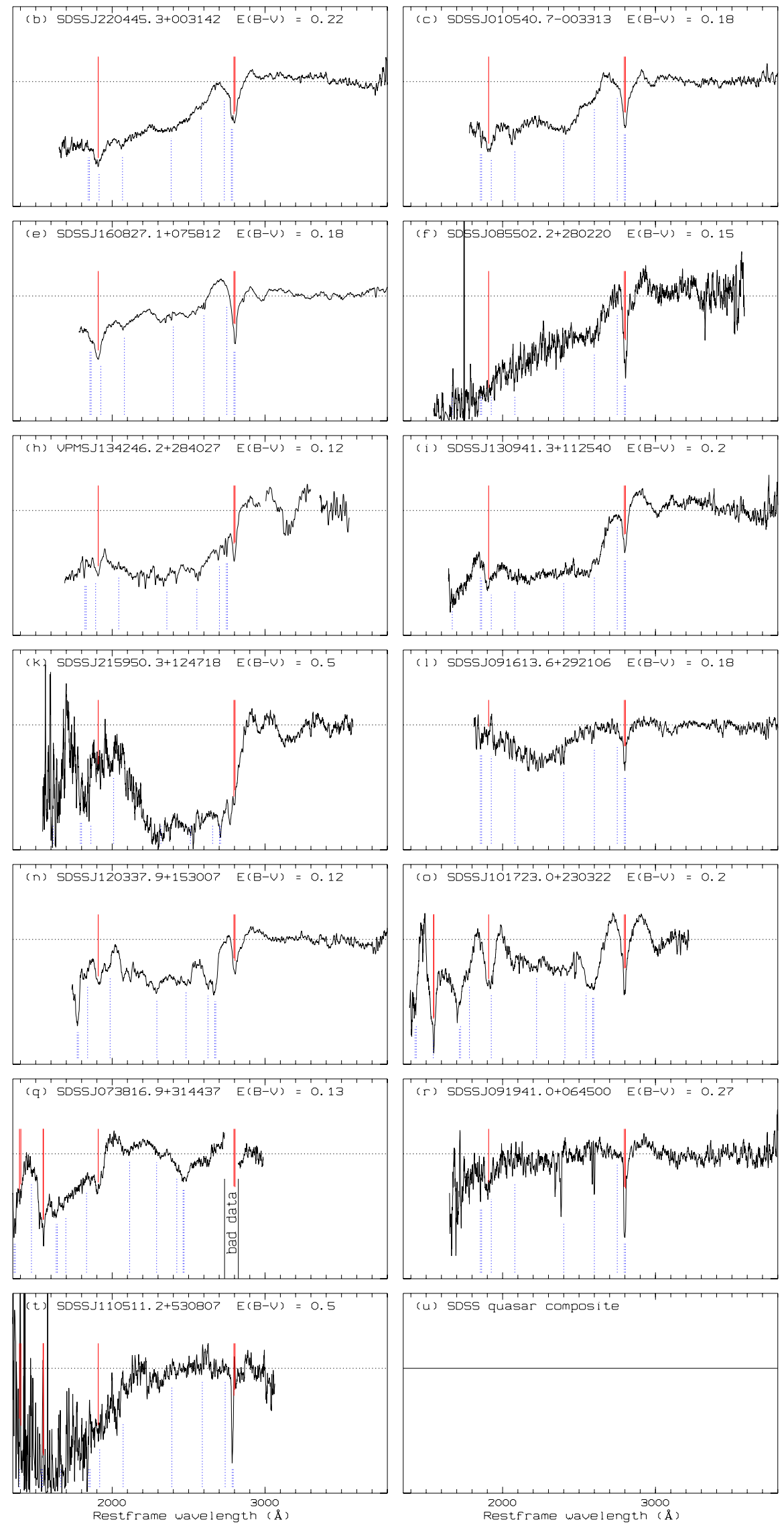
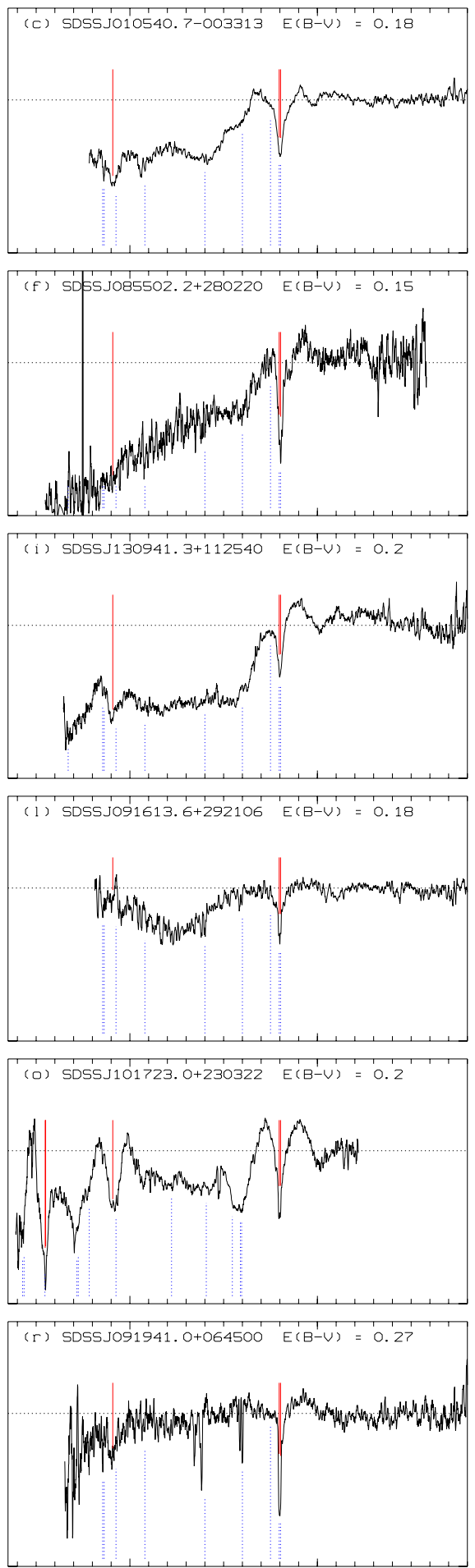

Fig. 13. As Fig. 12 but for the ratio of the dereddened spectra to the SDSS quasar composite.

\footnotetext{
Fig. 13. As Fig. 12 but for the ratio of the dereddened spectra to the SDSS quasar composite.
} 
H. Meusinger et al.: Kohonen-selected unusual SDSS quasars
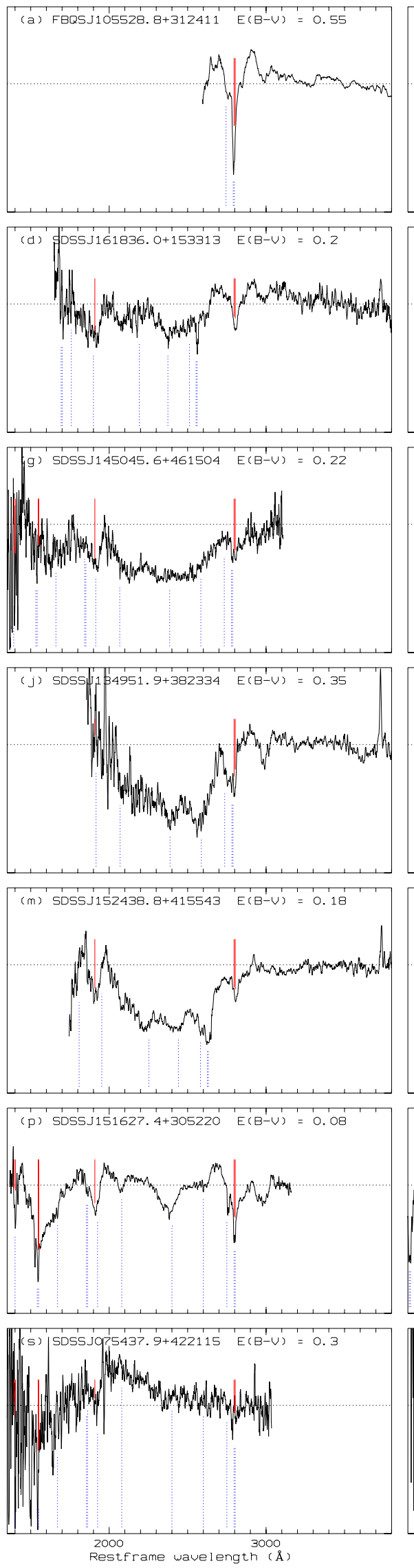
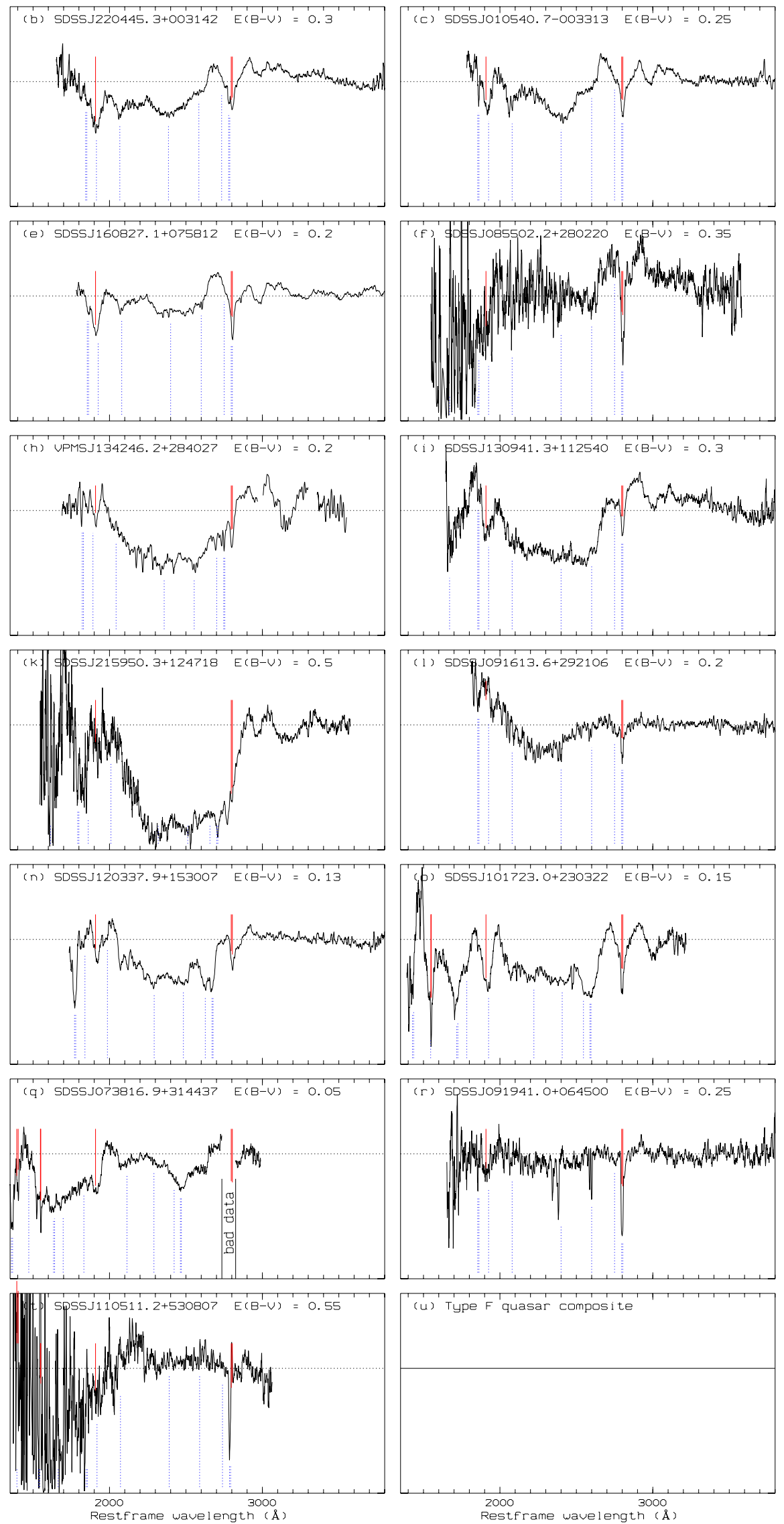

Fig. 14. As Fig. 12 but for the ratio of the dereddened spectra to the type F composite. 
the dereddening procedure with two modifications. First, the composite spectrum of strong iron emitters (type F) was used as reference instead of the SDSS quasar composite. Second, the individual quasar spectrum was fitted to the reference spectrum at either end. Figure 14 shows the ratios of the dereddened spectra to the reference spectrum. A good fit was achieved for SDSS J091940.97+064459.9. However, for most of the spectra the depression in the observed spectrum shortwards of $\mathrm{Mg}$ II was obviously not eliminated. Moreover, strong dereddening produces an unexplained bump at $2700 \AA$. As argued by Hall et al. (2002), Fe II emission may be present longwards of $\mathrm{Mg}$ II (near $3200 \AA$ ) but is not expected immediately shortwards of $\mathrm{Mg}$ II. In agreement with Hall et al., we conclude that the majority of the spectra cannot be explained by reddened strong iron emission alone.

For several objects in Figs. 12 to 14, the shape of the spectrum around $\mathrm{Mg}$ II resembles a broad double-line profile. The most interesting case is SDSS J101723.04+230322.1. Very broad double-shouldered $\mathrm{Mg}$ II emission, in combination with reddening and broad, shallow absorption troughs, is another possible scenario mentioned by Hall et al. (2002) to explain their two mysterious objects. As can be seen in Figs. 13 and 14, the spectrum of SDSS J101723.04+230322.1 cannot be understood as being caused by these double-shouldered line profiles alone or in combination with dust reddening, strong iron emission and associated narrow-line absorption. In addition, overlapping broad absorption troughs from various elements, in particular Fe II, are definitely needed to explain the complex shape of this spectrum. Remarkably, the apparent double-line profile in SDSS J101723.04+230322.1 is seen not only in the Mg II line but also for C III] and C IV. Only a few quasars have been reported so far to definitely display this structure in other lines than $\mathrm{H} \alpha$ and $\mathrm{H} \beta$ (Halpern et al. 2001; Strateva et al. 2003; Luo et al. 2009; Chornock et al. 2010). The selection of double-peaked AGNs has been primarily based on Balmer lines (Halpern et al. 2001; Eracleous et al. 2004). Ultraviolet observations have shown that their high-ionisation lines (e.g., C Iv) frequently lack doublepeaked profiles. However, double-peaked AGNs appear to be rather inhomogeneous as a class, thus we feel that this difference does not conclusively rule out the double-shouldered hypothesis for SDSS J101723.04+230322.1. It would be interesting to study the line profiles of the Balmer lines via infrared spectroscopy.

Most of the quasars in Table 8 are FIRST sources, but radio-weak, i.e. close to the threshold between radio-quiet and radio-loud quasars. The mean radio loudness of the seven radiodetected mysterious objects is $\left\langle R_{i}\right\rangle=0.97 \pm 0.28$. Among the three new mysterious objects, only SDSS J160827.08+075811.5 was detected by FIRST. SDSS J085502.20+280219.6 is 2.6 mag fainter in $i$ than SDSS J160827.08+075811.5 (and a FIRST flux as small as $0.2 \mathrm{mJy}$ thus still corresponds to $R_{i}=0.4$ ). The third quasar, SDSS J215950.30+124718.4, is not in the FIRST area. The mean radio loudness parameter of the 15 FIRST sources in Table 8 is $\left\langle R_{i}\right\rangle=1.02( \pm 0.41)$. The strongest radio source, SDSS J091940.97+064459.9, was classified by Plotkin et al. (2008) as a lower-confidence BL Lac object. None of the objects from Table 8 could be identified in the ROSAT All-Sky Survey Faint Source Catalog (Voges et al. 2000). Weak X-ray emission is a characteristic property of BAL quasars and is usually attributed to strong X-ray absorption, which enables and enhances the acceleration of the BAL outflow driven by radiation pressure (Murray \& Chiang 1998; Proga et al. 2000; Gallagher et al. 2006; Gibson et al. 2009; Streblyanska et al. 2010).

\section{Summary and conclusions}

We have compiled a catalogue of unusual quasars from the unprecedented spectroscopic database of the SDSS DR7. Unusual quasars were selected from $\sim 10^{5}$ spectra with $z=0.6$ to 4.3 classified as quasars by the SDSS spectroscopic pipeline. The selection method is essentially a combination of the power of Kohonen's (Kohonen 1982, 2001) SOMs and the detailed visual inspection of the selected spectra. We paid particular attention to reject contaminants, i.e. spectra from rare spectral types of other astrophysical objects (e.g., white dwarfs and hybrid spectra of two objects within the fibre aperture). Proper motions were checked first by cross-correlating our sample with the PPMXL catalogue (Röser et al. 2010), which contains $\sim 90 \%$ of the selected objects. In addition, and more importantly, we used all available multi-epoch positions from different sky surveys to estimate the proper motions of 121 selected sources. The results of the pm determination led to the rejection of seven objects from the quasar sample.

The final catalogue contains 1005 quasars, which were classified into 7 different types: (A) LoBALs and unusual BALs (21\%), (B) strong normal BALs, HiBALs (21\%), (C) red quasars (12\%), (D) quasars which appear red shortwards of $3000 \AA$ only (15\%), (E) weak-line quasars (18\%), (F) strong iron emitters $(11 \%)$, and $(\mathrm{M})$ miscellaneous. Our approach was primarily aimed at striking outliers and the catalogue is expected to be largely complete in this respect. However, the selection is not based on sharply defined quantitative criteria and the selected sample is thus incomplete in a quantifiable sense. Nevertheless, the compilation is expected to be very useful for studying relations between the various types of spectral peculiarities or selecting particularly interesting individual objects for detailed investigations.

The analysis of this sample yields the following conclusions for the main types A to F:

- The arithmetic median composite spectra (Sect. 5.5) clearly differ from type to type and from the SDSS composite spectrum of normal quasars. For type A, the huge spectrum-tospectrum variation reflects a high level of diversity. The spectral peculiarity, measured by the mean square deviation of the individual spectrum from the SDSS composite, is on average largest for types C,D, and A and smallest for type E. Unusual BALs are frequently accompanied by red continua and strong iron emission.

- We combined the SDSS spectra with the fluxes from 2MASS to compute geometric mean spectra with significantly improved wavelength coverage (Sect.5.5). These composite spectra were used to estimate the intrinsic reddening (Sect. 5.6). In agreement with previous studies, we found that BAL quasars have, on average, significantly redder continua than normal quasars with $E(B-V) \approx 0.1 \mathrm{mag}$ for type B adopting SMC extinction. The unusual BAL quasars (type A), which are mostly LoBALs, are even redder with $E(B-V) \approx 0.18 \mathrm{mag}$. The decline in the dereddened type A composite in the UV is ascribed to the combined effect of the UV absorption troughs. The strongest mean reddening is found for the type $\mathrm{C}$ with $E(B-V) \approx 0.38 \mathrm{mag}$. On the other side, weak-line quasars are on average insignificantly redder than normal $(E(B-V) \approx 0)$.

- For the quasars with substantial reddening in the UV only (type D), the continuum slope of the SDSS quasar composite could be fitted only with an extinction curve that is steeper in the UV than for the SMC (Sect. 5.6). 
- After the (statistical) correction for intrinsic extinction, the KS test for the absolute magnitudes yielded that all six types are on average more luminous than comparison samples of normal quasars (Sects. 5.2 and 5.6).

- The fraction of quasars with radio detections increases with the spectral peculiarity. This reflects most likely a selection bias: a substantial fraction of the quasars with very unusual spectra were selected for follow-up spectroscopy because they are radio sources. On the other hand, unusual BALs (as well as quasars with strong iron emission) tend not to have high radio luminosities. A similar, though not statistically significant trend was found for the red quasars. It is thus tempting to speculate that there exists a larger population of unusual quasars that have not yet been discovered simply because they are too faint radio sources (Sect. 5.3).

- To estimate the impact of foreground galaxies along the line of sight on the spectral peculiarities, we have checked both the SDSS images and spectra for finger prints of such intervening matter (Sect.5.7). About $1 \%$ of the quasar images show nearby (usually faint) structures and for $\sim 8 \%$ we detected narrow absorption lines that are distinct from the quasar lines. There are at least two quasars in the sample where the spectral peculiarity is clearly caused by foreground galaxies. Moreover, two of nine high- $z$ quasars identified on images from the Hubble Space Telescope (HST) show extended structures very close to the sightline (Appendix A). Nevertheless, we did not find any conclusive evidence that the sample properties are significantly affected by the extragalactic foreground.

- Our sample contains several objects with very peculiar spectra, among them FeLoBAL quasars with large numbers of narrow absorption troughs or extremely wide overlapping troughs. The most spectacular example of the latter type is SDSS J094317.59+541705.1 at $z=2.22$ where the continuum is strongly depressed from $\mathrm{Mg}$ II at red edge of the spectrum all the way down to Ly $\alpha$ at the blue edge (Sect. 6.1).

- We constructed a small sample of nine quasars with spectral properties similar to the two "mysterious" objects discovered by Hall et al. (2002) and another 11 more or less similar quasars (Sect. 6.2). The majority (75\%) of these objects are radio sources, but radio-weak $\left(R_{i} \approx 1\right)$. Both moderate reddening, FeLoBAL features, and strong Fe emission were frequently indicated. This combination may explain the shape of the continuum with the characteristic drop-off shortwards of the position of the $\mathrm{Mg}$ II line but does not account for the lack of typical quasar emission lines and the shape of the spectrum around the position of the $\mathrm{Mg}$ II line. Broad doublepeaked emission, in addition to other effects, is an attractive idea (Hall et al. 2002). Double-shouldered Mg II emission seems to be indicated in some spectra. The best case is SDSS J101723.04+230322.1 where similar profiles are, however, seen as well around $\mathrm{C}_{\mathrm{IV}}$ and $\mathrm{C}$ III], which is quite unusual for the known double-peaked quasars. Moreover, we have also presented a few quasars where the continuum dropoff appears at much shorter wavelengths, which seems difficult to explain in this way. A satisfactory explanation of these puzzling spectra remains a challenge.

Acknowledgements. We thank the referee, Patrick B. Hall, for providing many constructive comments and suggestions that significantly improved this paper. This research is based on the Sloan Digital Sky Survey (SDSS). Funding for the SDSS and SDSS-II has been provided by the Alfred P. Sloan Foundation, the Participating Institutions (see below), the National Science Foundation, the National Aeronautics and Space Administration, the US Department of Energy, the Japanese Monbukagakusho, the Max Planck Society, and the Higher Education Funding Council for England. The
SDSS Web site is http://www.sdss.org/. The SDSS is managed by the Astrophysical Research Consortium (ARC) for the Participating Institutions. The Participating Institutions are: the American Museum of Natural History, Astrophysical Institute Potsdam, University of Basel, University of Cambridge (Cambridge University), Case Western Reserve University, the University of Chicago, the Fermi National Accelerator Laboratory (Fermilab), the Institute for Advanced Study, the Japan Participation Group, the Johns Hopkins University, the Joint Institute for Nuclear Astrophysics, the Kavli Institute for Particle Astrophysics and Cosmology, the Korean Scientist Group, the Los Alamos National Laboratory, the Max-Planck-Institute for Astronomy (MPIA), the MaxPlanck-Institute for Astrophysics (MPA), the New Mexico State University, the Ohio State University, the University of Pittsburgh, University of Portsmouth, Princeton University, the United States Naval Observatory, and the University of Washington. This publication makes use of data products from the Widefield Infrared Survey Explorer, which is a joint project of the University of California, Los Angeles, and the Jet Propulsion Laboratory/California Institute of Technology, funded by the National Aeronautics and Space Administration. This research is based also on observations with the NASA/ESA Hubble Space Telescope, and obtained from the Hubble Legacy Archive, which is a collaboration between the Space Telescope Science Institute (STScI/NASA), the Space Telescope European Coordinating Facility (ST-ECF/ESA) and the Canadian Astronomy Data Centre (CADC/NRC/CSA). Finally, this research has made use of the SIMBAD database, operated at CDS, Strasbourg, France.

Note added in proof After this paper has been accepted for publication, we became aware of the paper by Plotkin et al. (2010) on optically selected BL Lac candidates from the SDSS. As a side-product, these authors discuss a small sample of serendipitously recovered/discovered higher-redshift objects that show extreme drop-offs in their continua bluewards of restframe $2800 \AA$. We realised that eight out of their nine objects are part of our sample of mysterious and related objects. Among them are four objects (SDSS J160827.08+075811.5, SDSS J161836.09+153313.5, SDSS J134951.93+382334.1, and SDSS J215950.30+124718.4) which were supposed to be newly discovered by the present study.

\section{Appendix A: Images of unusual quasars in the SDSS and in the Hubble Legacy Archive}

Unusual spectral properties can be the result of a positional coincidence with a foreground object in combination with gravitational lensing (Irwin et al. 1998; Chartas 2000). We used the SDSS Explorer and found that for $\sim 20 \%$ of the quasars, with only slight fluctuations from type to type, additional objects are seen within $12^{\prime \prime} \times 12^{\prime \prime}$ fields centred on the quasar. A substantial fraction is expected to be faint stars from our galaxy. For $1.3 \%$ of the quasars, we found another object closer than $\sim 2^{\prime \prime}$ or extended structures that likely overlap the line of sight towards the quasar. In this case, the fraction is largest for type $\mathrm{C}$ (3.4\%), but the samples are much too small for a statistical analysis. These 13 quasars are listed in Table 6. Among them are the two known lensed quasars SDSS J081959.80+535624.2 (Inada et al. 2010) and SDSS J090334.94+502819.3 (Johnston et al. 2003). The SDSS image of SDSS J081959.80+535624.2 shows a double structure with a separation $\lesssim 4^{\prime \prime}$. The spectrum indicates that there is an absorption line system at $z_{\mathrm{abs}}=0.294$. SDSS J090334.94+502819.3 was classified as an unusual quasar of type $\mathrm{C}$ because of its red continuum between Ly $\alpha$ and C IV. In addition, the $\mathrm{C}$ IV emission line is absorbed at the red wing. However, as demonstrated by Johnston et al. (2003), decomposing the spectrum into a luminous red galaxy component at $z=0.388$ and the background quasar component yields a normal quasar spectrum. Another interesting case is the quasar SDSS J024230.65-000029.7, which is seen through an outer spiral arm of NGC 1068.

One of us (B.K.) has performed a systematic search of the Hubble Legacy Archive for images from the HST covering quasars from our catalogue. Nine detections were found 


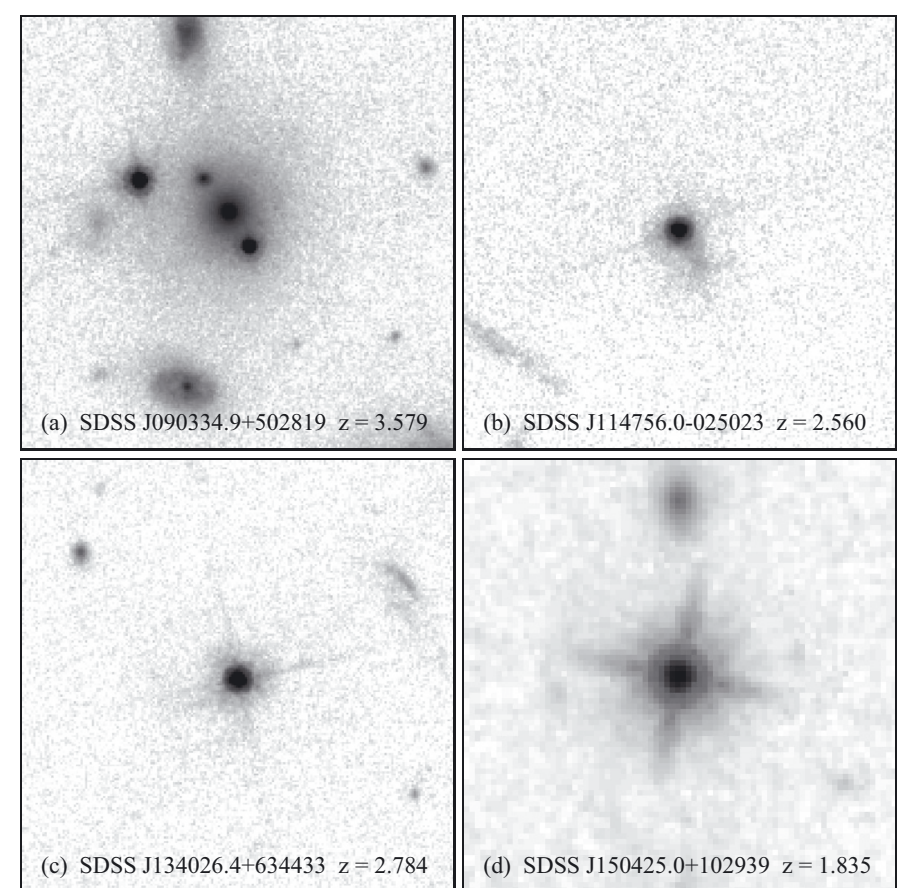

Fig. A.1. $10^{\prime \prime} \times 10^{\prime \prime}$ cutouts from the HST images of four quasars (unresolved objects close to the centre) showing extended structures within 5" from the line of sight (a) to c): ACS, d): WFC3).

Table A.1. Unusual quasars on HST images.

\begin{tabular}{lcccc}
\hline \hline SDSS J & $z$ & $T$ & Structure & Ref. \\
\hline $005006.34-005319.3$ & 4.347 & B & $\ldots$ & \\
$090334.94+502819.3$ & 3.579 & C & gal. group & 1 \\
$114756.00-025023.4$ & 2.560 & C & faint nearby & 2 \\
$122622.03+662018.0$ & 3.874 & B & $\ldots$ & \\
$134026.43+634433.1$ & 2.784 & A & faint 5 $5^{\prime \prime}$ & \\
$150424.98+102939.1$ & 1.835 & E & gal $\sim 4^{\prime \prime}$ & \\
$155633.78+351757.3$ & 1.499 & A & $\ldots$ & \\
$173049.10+585059.5$ & 2.033 & A & $\ldots$ & \\
$220557.02+121239.7$ & 1.382 & E & $\ldots$ & \\
\hline
\end{tabular}

References. (1) Johnston et al. (2003); (2) Bentz et al. (2008).

(Table A.1). Two quasars clearly show extended structures close to the sightline (Fig. A.1). The gravitationally lensed quasar SDSS J090334.9+502819 mentioned above is seen through the inner region of a luminous featureless galaxy. The other object, SDSS J114756.0-025023 at $z=2.560$, is connected with a faint structure, which may be at the redshift of the quasar (Bentz et al. 2008). Both spectra show red continua of type $C$ and line $a b-$ sorption at $z_{\mathrm{abs}} \approx z_{\mathrm{em}}$. Extended structures are also seen around SDSS J134026.4+634433 and SDSS J150425.0+102939, although at somewhat larger distances of $\sim 4$ " from the line of sight. There are no clear cases of the signatures of foreground objects in the spectra of these quasars.

\section{References}

Abajas, C., Mediavilla, E., Muñoz, J. A., et al. 2007, ApJ, 658, 748 Abazajian, K. N., Adelman-McCarthy, J. K., Agüeros, M. A., et al. 2009, ApJS, 182,543

Adelman-McCarthy, J. K., Agüeros, M. A., Allam, S. S., et al. 2006, ApJS, 162, 38

Aihara, H., Allende Prieto, C., An, D., et al. 2011, ApJS, 193, 29

Allen, J. T., Hewett, P. C., Maddox, N., et al. 2011, MNRAS, 410, 860
Antonucci, R. 1993, ARA\&A, 31, 473

Bahcall, J. N., \& Spitzer, L. J. 1969, ApJ, 396, 20

Baldwin, J. 1977, ApJ, 214, 679

Bennert, N., Canalizo, G., Jungwirt, B., et al. 2008, ApJ, 677, 846

Becker, R. H., White, R. L., \& Helfand, D. J. 1995, ApJ, 450, 559

Becker, R. H., Gregg, M. D., Hook, I. M., et al. 1997, ApJ, 479, L93

Becker, R. H., White, R. L., Gregg, M. D., et al. 2000, ApJ, 538, 72

Bentz, M. C., Pogge, R. C., \& Osmer, P. S. 2008, AJ, 136, 498

Blackburne, J. A., Pooley, D., Rappaport, S., \& Schechter, P. L. 2011, ApJ, 729 34

Blaes, O., Hubeny, I., Agol, E., \& Krolik, J. H. 2001, ApJ, 563, 560

Boroson, T. A., \& Green, R. 1992, ApJS, 80, 109

Boroson, T. A., \& Meyers, K. A. 1992, ApJ, 397, 442

Bowen, D. V., \& Chelouche, D. 2011, ApJ, 727, 47

Brunner, R. J., Hall, P. B., Djorgovski, S. G., et al. 2003, AJ, 126, 53

Canalizo, G., \& Stockton, A. 2001, ApJ, 555, 719

Carballo, R., González-Serrano, J. I., Montenegro-Montes, F. M., et al. 2006 , MNRAS, 370, 1034

Carlsberg Meridian Catalog (CMC14) 2006, Copenhagen University Obs., Institute of Astronomy, Cambridge, UK, Real Instituto y Observatorio de la Armada en San Fernando

Carollo, D., Bucciarelli, B., Hodgkin, S. T., et al. 2006, A\&A, 448, 579

Chartas, G. 2000, ApJ, 531, 81

Chisholm, J. R., Dodelson, S., \& Kolb, E. W. 2003, ApJ, 596, 437

Chornock, R., Bloom, J. S., Cenko, S. B., et al. 2010, ApJ, 709, L39

Cutri, R. M., Nelson, B. O., Kirkpatrick, J. D., et al. 2001, ASP Conf. Ser., 232 78

Dai, X., Shankar, F., \& Sivakoff, G. R. 2008, ApJ, 672, 108

Dai, X., Shankar, F., \& Sivakoff, G. R. 2010, MNRAS, submitted [arXiv: $1004.0700 \mathrm{v} 1]$

Diamond-Stanic, A. M., Fan, X., Brandt, W. N., et al., 2009, ApJ, 699, 782

Doi, A., Kawagucchi, N., Kono, Y., et al. 2009, PASJ, 61, 1389

Eracleous, M., Halpern, J. P., Storchi-Bergmann, T., et al. 2004, in The Interplay among Black Holes, Stars and ISM in Galactic Nuclei, ed. T. StorchiBergmann, L. C. Ho, \& H. R. Schmitt (Cambridge Univ. Press), 229

Fan, X., Strauss, M. A., Gunn, J. E., et al., 1999, ApJ, 526, L57

Farrah, D., Urrutia, T., Lacy, M., et al. 2010, ApJ, 717, 868

Foltz, C., Wilkes, B., Weymann, R., et al. 1983, PASP, 95, 341

Francis, P. J., Hewett, P. C., Foltz, C. B., et al. 1991, ApJ, 373, 465

Gallagher, S. C., Brandt, W. N., Chartas, G., et al. 2006, ApJ, 644, 709

Gallagher S. C., Hines, D. C., Blaylock, M., et al. 2007, ApJ, 665, 157

Ganguly, R., \& Brotherton, M. S. 2008, ApJ, 672, 102

Gaskell, C. M. 1983, in Quasars and Gravitational Lenses, ed. J. P. Swings, University de Liege, Proc. 24th Liege Int. Astrophys. Colloq., 473

Gehrels, N. 1986, ApJ, 303, 336

Georgakakis, A., Clements, D. L., Bendo, G., et al. 2009, MNRAS, 394, 533

Gibson, R. R., Jiang, L., Brandt, W. N., et al. 2009, ApJ, 692, 758

Glikman, E., Helfand, D. J., White, R. L., et al. 2007, ApJ, 667, 673

Gosh, K. K., \& Punsly, B. 2007, ApJ, 661, L139

Greenstein, J. L., \& Matthews, T. A. 1963, AJ, 68, 279

Guyon, O., Sanders, D. B., \& Stockton, A. 2006, ApJS, 166, 89

Hall, P. B., \& Chajet, L. S. 2011, MNRAS, submitted [arXiv: 1105.1689]

Hall, P. B., Anderson, S. F., Strauss, M. A., et al. 2002, ApJS, 141, 267

Hall, P. B., Knapp, G. R., Richards, G. T., et al. 2004, in Multiwavelengths AGN Surveys, ed. R. Mújica, \& R. Maiolino (World Scientific Publishing), 247

Hall, P. B., Anosov, K., White, R. L., et al. 2011, MNRAS, 411, 2653

Halpern, J. P., Eracleous, M., Filippenko, A. V., et al. 1996, ApJ, 464, 704

Hambly, N. C., MacGillivray, H. T., Read M. A., et al. 2001, MNRAS, 326, 1279

Häring, N., \& Rix, H. W. 2004, ApJ, 604, 89

Hazard, C., McMahon, R. G., Webb, J. K., et al. 1987, ApJ, 323, 263

Heller, R., Dreizler, S., \& Ostensen, R. 2009, A\&A, 496, 191

Hernquist, L. 1989, Nature, 340, 687

Hewett, P. C., \& Foltz, C. B. 2003, AJ, 125, 1784

Hines, D. C., Schmidt, G. D., Gordon, K. D., et al. 2001, ApJ, 563, 512

Hopkins, P. F., Hernquist, L., Cox, T. J., et al. 2005, ApJ, 630, 705

Hopkins, P. F., Hernquist, L., Cox, T. J., et al. 2006, ApJS, 163, 1

Hryniewicz, K., Czerny, B., Nikolajuk, M., \& Kuraszkewicz, J. 2010, MNRAS, 404, 2028

Inada, N., Oguri, M., Shin, M.-S., et al. 2010, AJ, 140, 403

Irwin, M. J., Ibata, R. A., Lewis, G. F., et al. 1998, ApJ, 505, 529

Ivezić, Ž., Menou, K., Knapp, G. R., et al. 2002, AJ, 124, 2346

Jiang, P., Ge, J., Zhou, H., et al. 2011, ApJ, 732, 110

Johnston, D. E., Richards, G. T., Frieman, J. A., et al. 2003, AJ, 126, 2281

Just, D. W., Brandt, W. N., Shemmer, O., et al. 2007, ApJ, 665, 1004

Kacprzak, G. G., Churchill, C. W., Evans, J. E., et al. 2011, MNRAS, 416, 3118

Kaczmarczik, M. C., Richards, G. T., Mehta, S. S., \& Schlegel, D. J. 2009, AJ, 138,19

Kauffmann, G., \& Hähnelt, M. 2000, MNRAS, 311, 576 
Kauffmann, G., \& Heckman, T. M. 2009, MNRAS, 397, 135

Kennefick, J., \& Bursick, S. 2008, AJ, 136, 179

Kinney, A. L., Rivolo, A. R., \& Koratkar, A. P. 1990, ApJ, 357, 338

Kishimoto, M., Antonucci, R., Blaes, O., et al. 2008, Nature, 454, 492

Kohonen, T. 1982, Biological Cybernetics, 43, 49

Kohonen, T. 2001, Self-Organizing Maps, third edition (New York: Springer)

Komossa, S., Burwitz, V., Hasinger, G., et al. 2003, ApJ, 582, L15

Koratkar, A., \& Blaes, O. 1999, PASP, 111, 1

Kron, R. G., \& Chiu, L.-T. 1981, PASP, 93, 397

Lang, D., Hogg, D. W., Jester, S., \& Rix, H. W. 2009, AJ, 137, 4400

Laor, A. 1990, MNRAS, 246, 369

Laor, A., \& Davis, S. W. 2011, MNRAS, 417, 681

Lawrence, A., Warren, S. J., Almaini, O., et al. 2007, MNRAS, 379, 1599

Leipski, C., Haas, M., Meusinger, H., \& Siebenmorgen, R. 2008, ASP Conf. Ser., 381,422

Leighly, K. M., Halpern, J. P., Helfand, D. J., et al. 2001, AJ, 121, 2889

Leighly, K. M., Halpern, J. P., Jenkins, E. B., \& Casebeer, D. 2007, ApJS, 173, 1

Lípari, S., Sanchez, S. F., Bergmann, M., et al. 2009, MNRAS, 392, 1295

Luo, B., Brandt, W. N., Silverman, J. D., et al. 2009, ApJ, 695, 1227

Magorrian, J., Tremaine, S., Richstone, D., et al. 1998, AJ, 115, 2285

Martínez-Sansigre, A., Rawlings, S., Lacy, M., et al. 2006, MNRAS, 370, 1479

Massaro, E., Giommi, P., Leto, C., et al. 2009, A\&A, 495, 691

McDowell, J. C., Canizares, C., Elvis, M., et al. 1995, ApJ, 450, 585

McMahon, R. G., Irwin, M. J., \& Maddox, S. J. 2000, The APM-North Catalogue, Institute of Astronomy, Cambridge, CB3 OHA, UK

Ménard, B., Nestor, D. B., Turnshek, D. A., et al. 2008, MNRAS, 385, 1053

Meng, X.-M., Wu, H., Gu, Q.-S., et al. 2010, ApJ, 718, 928

Menou, K., Vanden Berk, D. E., Ivezić, Ž., et al. 2001, ApJ, 561, 645

Meusinger, H., Scholz, R.-D., Irwin, M. J., \& Lehmann, H. 2002, A\&A, 392, 851

Meusinger, H., Brunzendorf, J., \& Laget, M. 2003, Astron. Nachr., 324, 474

Meusinger, H., Froebrich, D., Haas, M., et al. 2005, A\&A, 433, L25

Meusinger, H., Hinze, A., \& de Hoon, A. 2011, A\&A, 525, A37

Montenegro-Montes, F. M., Mack, K.-H., Benn, C. R., et al. 2009, Astron. Nachr., 330, 157

Murray, N., \& Chiang, J. 1998, ApJ, 494, 125

Osmer, P. S., Porter, A. C., \& Green, R. F. 1994, ApJ, 436, 678

Pei, Y. C. 1992, ApJ, 395, 130

Plotkin, R. M., Anderson, S. F. Hall, P. B., et al. 2008, AJ, 135, 2453

Plotkin, R. M., Anderson, S. F., Brandt, W. N., et al. 2010, AJ, 139, 390

Polletta, M., Weedman, D., Hönig, S., et al. 2008, ApJ, 675, 960

Proga, D., Stone, J. M., \& Kallman, T. R. 2000, ApJ, 543, 686

Reichard, T. A., Richards, G. T., Hall, P. B., et al. 2003, AJ, 126, 2594

Richards, G. T., Fan, X., Newberg, H. J., et al. 2002, AJ, 123, 2945

Richards, G. T., Hall, P. B., Vanden Berk, D. E., et al. 2003, AJ, 126, 1131

Richards, G. T., Kruczek, N. E., Gallagher, S. C., et al. 2011, AJ, 141, 167

Röser, S., Demleitner, M., \& Schilbach, E. 2010, AJ, 139, 2440

Sameshima, H., Kawara, K., Matsuoka, Y., et al. 2011, MNRAS, 410, 1018

Sandage, A. 1965, ApJ, 141, 1560

Sandage, A., \& Luyten, W. J. 1969, ApJ, 155, 913
Sanders, D. B., Soifer, B. T., Elias, J. H., et al. 1988, ApJ, 325, 74

Schmidt, M. 1963, Nature, 197, 1040

Schmidt, G. D., Liebert, J., Harris, H. C., et al. 1999, ApJ, 512, 916

Schmidt, G. D., Szkody, P., Henden, A., et al. 2007, ApJ, 654, 521

Schneider, D. P., Richards, G. T., Hall, P. B., et al. 2010, AJ, 139, 2360

Scholz, R. D., Meusinger, H., \& Irwin, M. J. 1997, A\&A, 325, 457

Shabala, S. S., Kaviray, S., \& Silk, J. 2011, MNRAS, 413, 2815

Shakura, N. I., \& Sunyaev, R. A. 1973, A\&A, 24, 337

Shankar, F., Dai, X., \& Sivakoff, G. R. 2008, ApJ, 687, 859

Shemmer, O., Brandt, W. N., Anderson, S. F., et al. 2009, ApJ, 696, 580

Shemmer, O., Trakhtenbrot, B., Anderson, S. F., et al. 2010, ApJ, 722, L152

Shields, G. A. 1978, Nature, 272, 706

Siegel, S., \& Castellan, N. J. 1988, Nonparametric Statistics for the Behavioural

Sciences (New York : McGraw-Hill, Inc.)

Skrutskie, M. F., Cutri, R. M., Stiening, R., et al. 2006, AJ, 131, 1163

Sprayberry, D., \& Foltz, C. B. 1992, ApJ, 390, 39

Springel, V., Di Matteo, T., \& Hernquist, L. 2005, MNRAS, 361, 776

Steidel, C. C., Dickinson, M., Meyer, D. M., et al. 1997, ApJ, 480, 568

Surdej, J., \& Hutsemekers, D. 1987, A\&A, 177, 42

Szkody, P., Homer, L., Chen, B., et al. 2004, AJ, 128, 2443

Strateva, I. V., Strauss, M. A., Schneider, D. P., et al. 2003, AJ, 126, 1720

Streblyanska, A., Barcons, X., Carrera, F. J., \& Gil-Merino, R. 2010, A\&A, 515,

Sulentic, J. W., Dultzin-Hacyan, D., \& Marziani, P. 2007, Rev. Mex. Astron. Astrofis., 28, 83

Tang, S., \& Grindlay, J. 2009, ApJ, 704, 1189

Taniguchi, Y. 2003, ASP Conf. Ser., 289, 353

Trump, J. R., Hall, P. B., Reichard, T. A., et al. 2006, ApJS, 165, 1

Tsuzuki, Y., Kawara, K., Yoshi, Y., et al. 2006, ApJ, 650, 57

Ultsch, A., \& Siemon, H. P. 1990, in Proc. Int. Neural Network Conf. (Dordrecht: Kluwer), 305

Urrutia, T., Becker, R. H., White, R. L., et al. 2009, ApJ, 698, 1095

Urry, C. M., \& Padovani, P. 1995, PASP, 107, 803

Vanden Berk, D. E., Richards, G. T., Bauer, A., et al. 2001, AJ, 122, 549

Vladilo, G., Prochaska, J. X., \& Wolfe, A. M. 2008, A\&A, 478, 701

Voges, W., Aschenbach, B., Boller, T., et al. 2000, IAU Circ., 7432, 1

Voit, G. M., Weymann, R. J., \& Korista, K. T. 1993, ApJ, 413, 95

Webster, R. L., Francis, P. J., Peterson, B. A., et al. 1995, Nature, 375, 469

Weymann, R. J., Morris, S. L., Foltz, C. B., et al. 1991, ApJ, 373, 23

White, R. L, Becker, R. H., \& Gregg, M. D. 2000, ApJS, 126, 133

White, R. L., Helfand, D. J., Becker, R. H., et al. 2003, AJ, 126, 706

Wild, V., Hewett, P. C., \& Pettini, M. 2006, MNRAS, 367, 211

Wilhite, B. C., Vanden Berk, D. E., Kron, R. G., et al. 2005, ApJ, 633, 638

Wright, E. L., Eisenhardt, P. R. M., Mainzer, A. K., et al. 2010, AJ, 140, 1868

Wu, J., Brandt, W. N., Hall, P. B., et al. 2011, ApJ, 736, 28

Wu, J., Brandt, W. N., Anderson, S. F., et al. 2012, ApJ, 747, 10

Yonehara, A., Hirashita, H., \& Richter, P. 2008, A\&A, 478, 95

York, D. G., Khare, P., Vanden Berk, D., et al. 2006, MNRAS, 367, 945

Zhang, S., Wang, T.-G., Wang, H., et al. 2010, ApJ, 714, 367

Zheng, W., Kriss, G. A., Telfer, R. C., et al. 1997, ApJ, 475, 469 